Supporting Information

\title{
Visible Light-mediated Photochemical Reactions of 2-(2'-
}

\author{
Alkenyloxy)cycloalk-2-enones \\ Raphaela Graß1, Christian Jandl, Thorsten Bach* \\ Department Chemie and Catalysis Research Center (CRC), Technische Universität München, \\ Lichtenbergstr. 4, 85747 Garching, Germany
}

\section{Corresponding Author}

"Email: thorsten.bach@ ch.tum.de

\section{Contents}

1. Sensitizers and Catalyst Loadings Tested in Initial Studies...................................................... S2

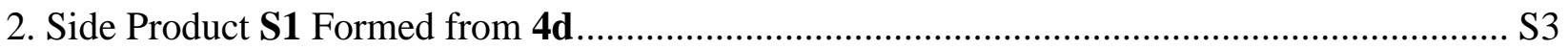



4. Important NOE Contacts Obtained from NOESY Spectra of Compounds $\mathbf{8 b}, \mathbf{9 b - e}, \mathbf{1 1}, \mathbf{1 3}$ and

16a-c S6

5. $420 \mathrm{~nm}$ Fluorescent Light Tube Emission Spectrum ........................................................... S8

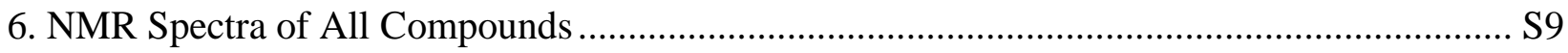

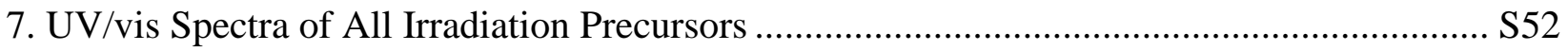

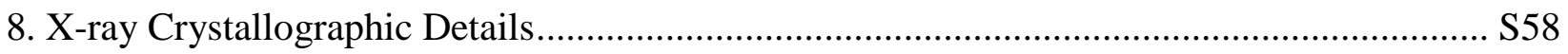

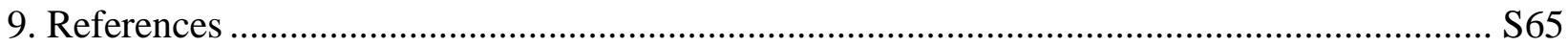


1. Sensitizers and Catalyst Loadings Tested in Initial Studies

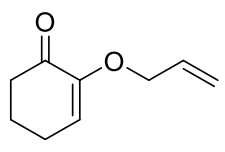

1 a

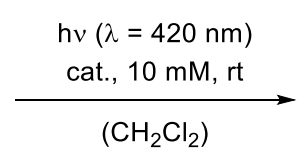

$\left(\mathrm{CH}_{2} \mathrm{Cl}_{2}\right)$

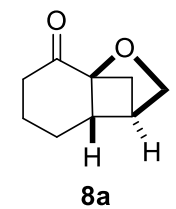

\begin{tabular}{|c|c|c|c|c|c|}
\hline$\#$ & Kat. & [mol\%] & $\mathrm{E}_{\mathrm{T}}[\mathrm{kJ} / \mathrm{mol}]$ & $\mathrm{T}[\mathrm{h}]$ & $\mathrm{Y}[\%]$ \\
\hline 1 & $-*$ & - & - & 2.5 & 35 \\
\hline 2 & Benzophenone* & 10 & $290^{1}$ & 2.0 & 61 \\
\hline 3 & Txt & 10 & $268^{2}$ & 2.5 & 73 \\
\hline 4 & Txt & 5.0 & 268 & 2.5 & 72 \\
\hline 5 & Txt & 2.5 & 268 & 2.5 & 70 \\
\hline 6 & ${ }^{\mathrm{F}}$ Irpic & 2.5 & $256^{3}$ & 2.5 & 81 \\
\hline 7 & $\operatorname{Ir}(\mathrm{pFppy})_{3}$ & 2.5 & $245^{4}$ & 3.0 & 69 \\
\hline 8 & $\operatorname{Ir}(\text { ppy })_{3}$ & 2.5 & $231^{4}$ & 5.0 & 85 \\
\hline 9 & $\mathrm{Ru}(\mathrm{bpy})_{3}\left(\mathrm{PF}_{6}\right)_{2}$ & 2.5 & $193^{5}$ & 27 & (rsm) \\
\hline
\end{tabular}

* irradiation performed at $350 \mathrm{~nm}$ 


\section{Side Product $\mathbf{S 1}$ Formed from $\mathbf{4 d}$}

In analogy to compound 2d: $\mathrm{KOH}(85 \mathrm{wt} \%, 283 \mathrm{mg}, 4.28 \mathrm{mmol}, 1.0$ equiv.) was dissolved in methallylic alcohol (1.5 mL, $1.29 \mathrm{mg}, 17.9 \mathrm{mmol}, 4.2$ equiv.). A solution of epoxide $\mathbf{4 d}$ (603 mg, $4.30 \mathrm{mmol}, 1.0$ equiv.) in methallylic alcohol (1.0 mL, $0.851 \mathrm{mg}, 11.8 \mathrm{mmol}, 2.8$ equiv.) was then added dropwise and the reaction mixture was stirred at room temperature over night. Subsequently the reaction mixture was diluted with $15 \mathrm{~mL} \mathrm{CH} \mathrm{Cl}_{2}$ and quenched by the addition of $15 \mathrm{~mL} \mathrm{H}_{2} \mathrm{O}$. The layers were separated and the aqueous layer was extracted with $\mathrm{CH}_{2} \mathrm{Cl}_{2}$ $(3 \times 30 \mathrm{~mL})$. The combined organic layers were washed with brine $(1 \times 150 \mathrm{~mL})$, dried over $\mathrm{Na}_{2} \mathrm{SO}_{4}$, filtrated and the solvent as well as the remaining volatiles were removed under reduced pressure. The irradiation precursor $\mathbf{2 d}$ was dissolved in a minimum of $\mathrm{Et}_{2} \mathrm{O}$ and subjected to flash column chromatography. The remaining colorless solid was filtered off. It is insoluble in $\mathrm{Et}_{2} \mathrm{O}$ and poorly soluble in $\mathrm{CH}_{2} \mathrm{Cl}_{2}$. Single crystals were obtained from a saturated solution of $\mathbf{S 1}$ in $\mathrm{CH}_{2} \mathrm{Cl}_{2}$. The structure and relative configuration of $\mathbf{S 1}$ were determined by data obtained from SC-XRD measurements.

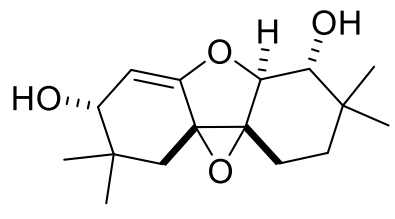

S1

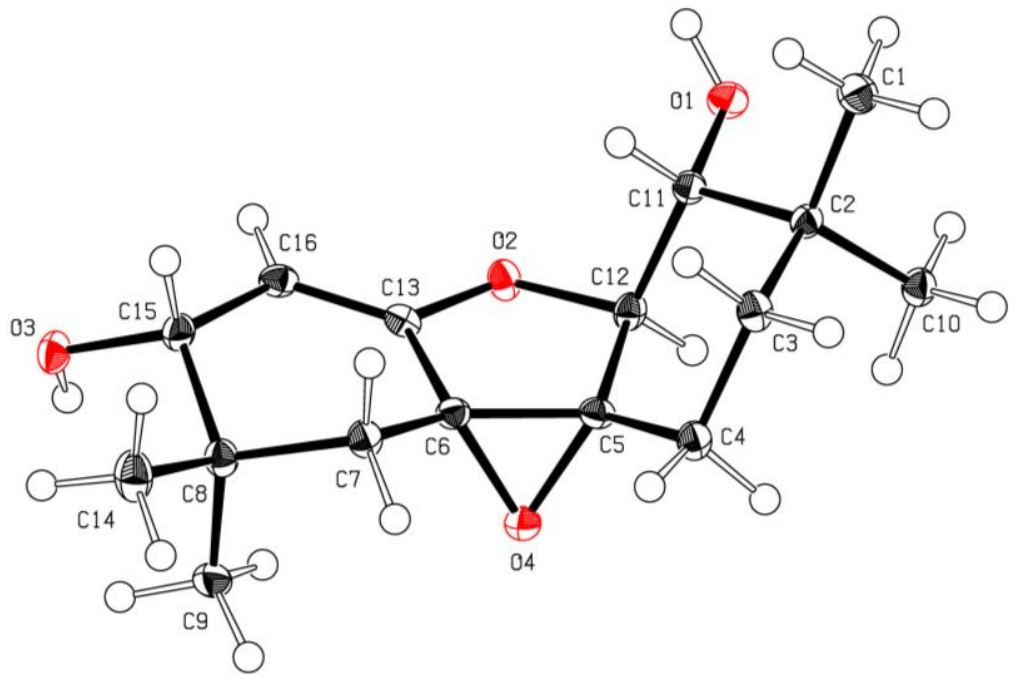

FIGURE S1. Structure of side product S1 obtained by dimerization of epoxide 4d. Ellipsoids in the ORTEP structure are displayed at the $50 \%$ probability level. 

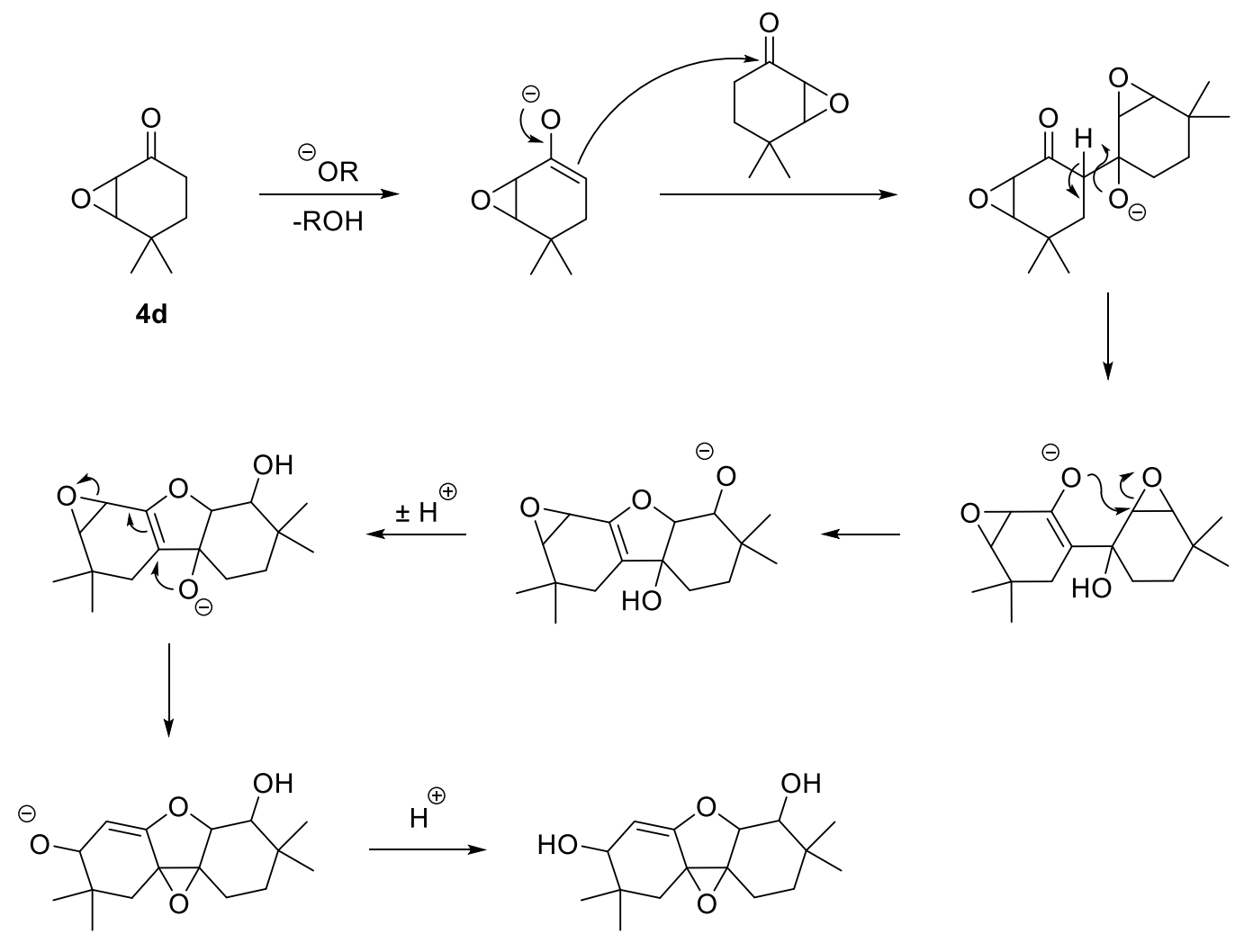

s1

SCHEME S1. Mechanistic proposal for the formation of S1.

\section{Phosphorescence Spectrum of 1a}

Luminescence Measurements were performed on a Horiba Scientific FluoroMax-4P instrument equipped with a continuous Xe source for steady state measurements and a Xe flashlight source for the observation of phosphorescence spectra. Fluorescence spectra were recorded at ambient conditions in 10×10 mm quartz cuvettes (Hellma, Suprasil). Luminescence spectra at cryogenic conditions $(77 \mathrm{~K})$ were recorded in quartz tubes (inner $\emptyset=4 \mathrm{~mm}$ ) in a small quartz Dewar vessel which was filled with liquid nitrogen. All solutions were handled under dry nitrogen and degassed (15 min in an ultrasoundbath) to exclude oxygen as triplet quencher. A longpass filter (Schott Advanced Optics,WG300 or Hebo Spezialglass, UV280) was introduced in the emission- 
beam path to prevent the excitation light to pass the emission monochromator at higher order wavelengths where necessary.

a)

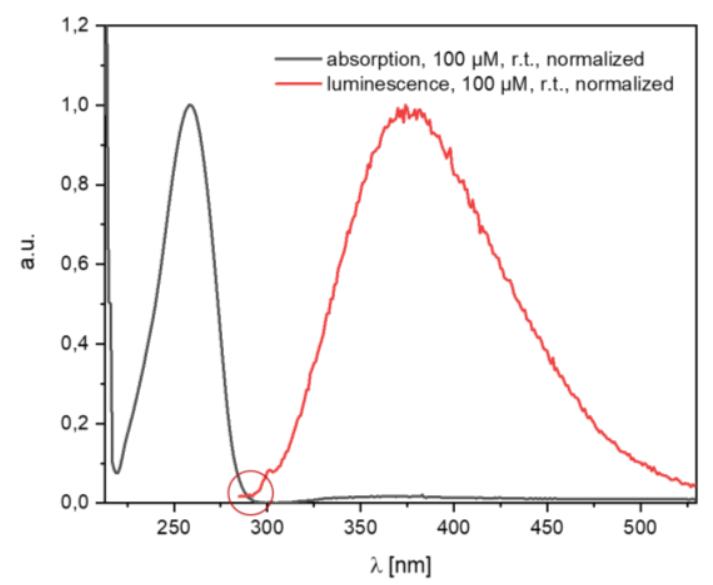

b)

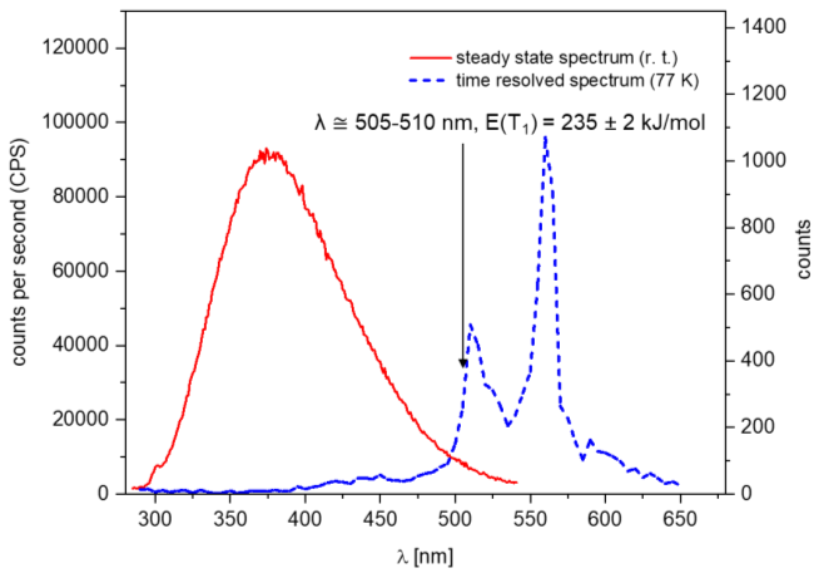

FIGURE S2. (a) Recorded UV/VIS of $1 \mathbf{a}$ in $\mathrm{CH}_{2} \mathrm{Cl}_{2}(\mathrm{c}=100 \mu \mathrm{M})$, normalized to $\mathrm{A}_{259} \mathrm{~nm}$; recorded luminescence (UV280 filter glass applied) of $\mathbf{1 a}$ in $\mathrm{CH}_{2} \mathrm{Cl}_{2}(\mathrm{c}=100 \mu \mathrm{M})$ at ambient conditions, normalized to $\mathrm{I}_{374} \mathrm{~nm}$. (b) Steady state spectrum of $\mathbf{1 a}$ in $\mathrm{CH}_{2} \mathrm{Cl}_{2}(\mathrm{c}=100 \mu \mathrm{M})$ at ambient conditions (UV280 filter glass applied) given in counts per second (red, solid line); time resolved spectrum (WG300 filter glass applied) of $\mathbf{1 a}$ in $\mathrm{CH}_{2} \mathrm{Cl}_{2}(\mathrm{c}=100 \mu \mathrm{M})$ after $50 \mu$ s delay at $77 \mathrm{~K}$ in counts (blue, dashed line). 
4. Important NOE Contacts Obtained from NOESY Spectra of Compounds $\mathbf{8 b}, \mathbf{9 b}-\mathbf{e}, \mathbf{1 1}, \mathbf{1 3}$ and 16a-c
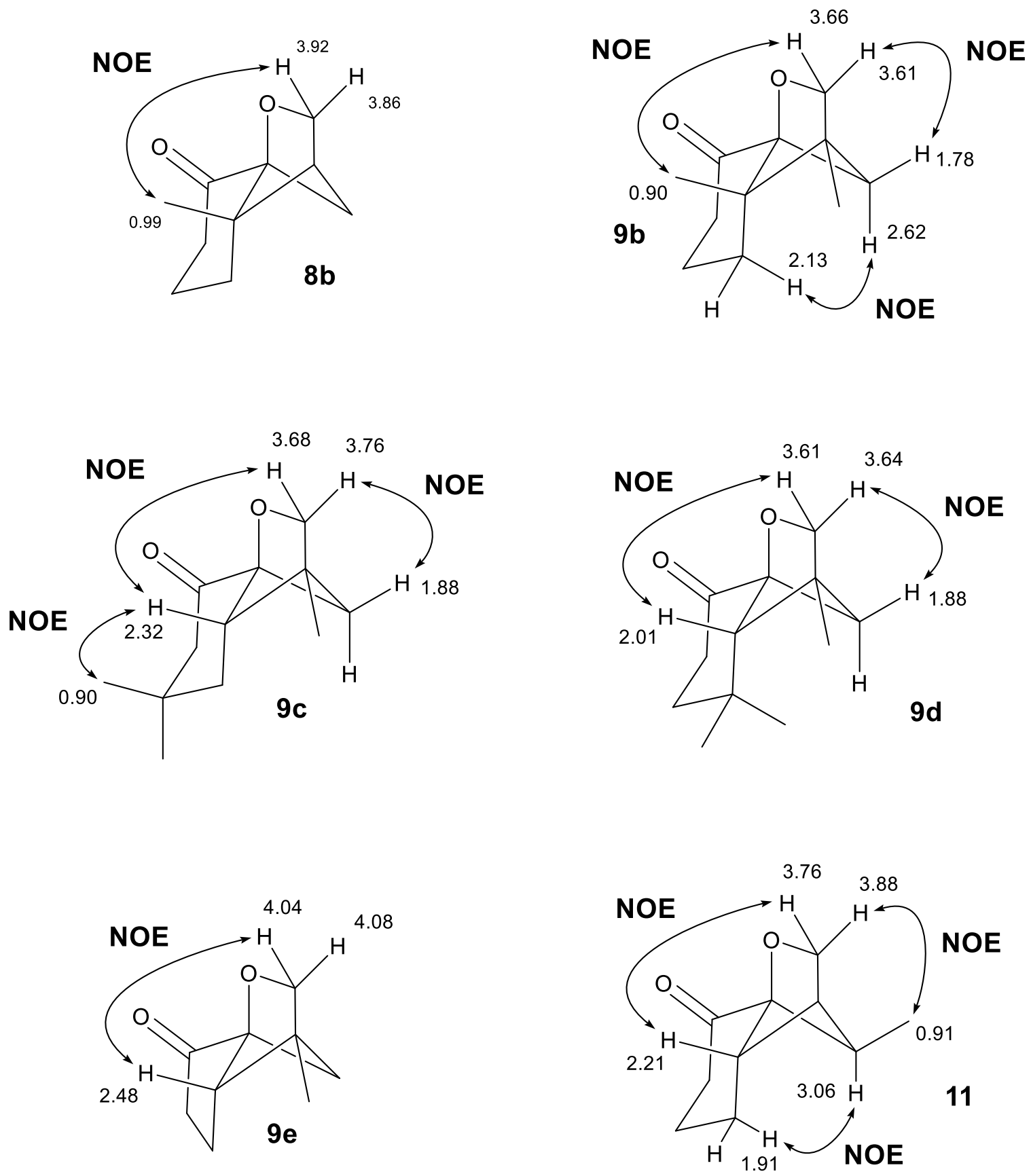

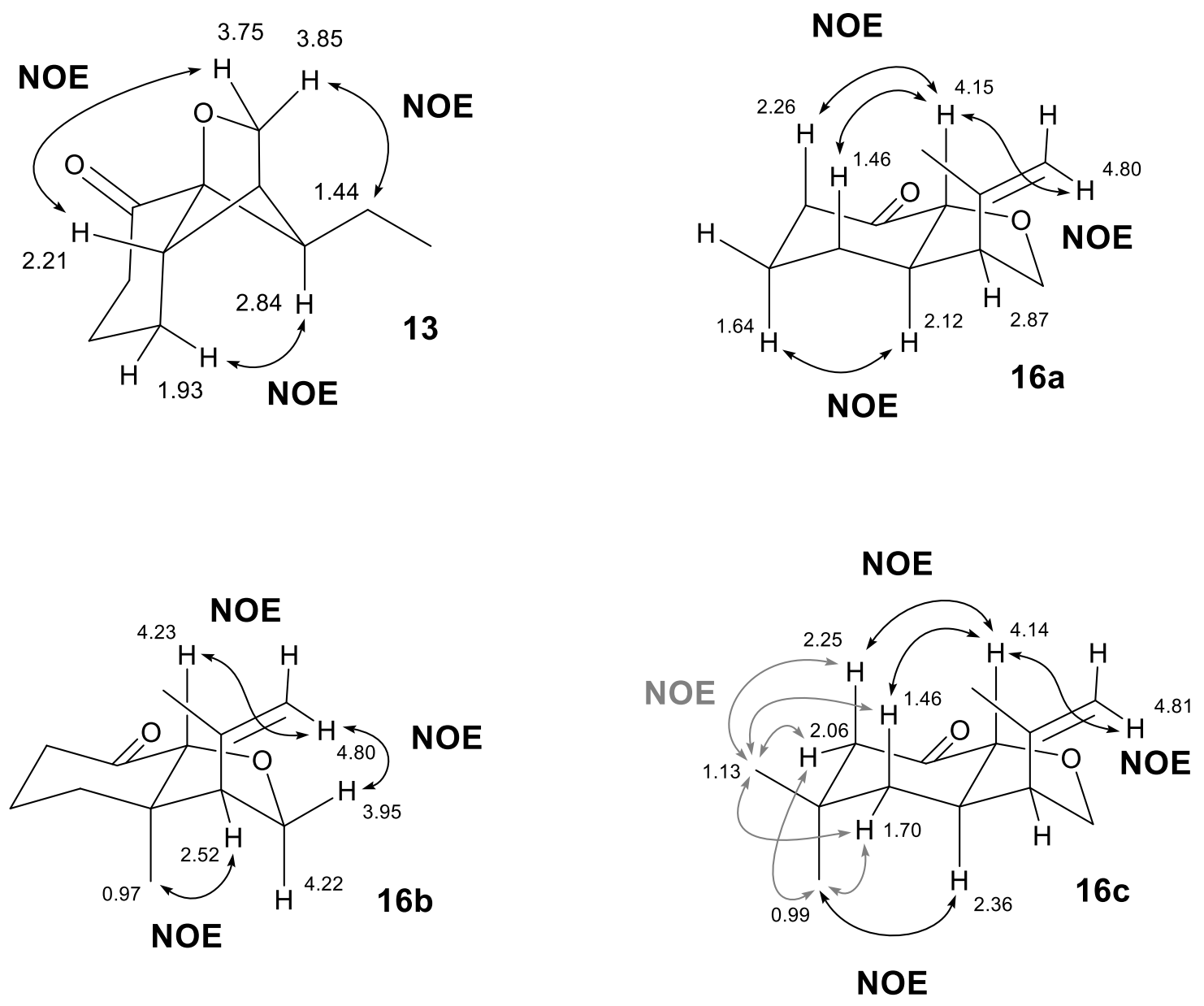
5. $420 \mathrm{~nm}$ Fluorescent Light Tube Emission Spectrum

Lehrstuhl OC 1 - TUM

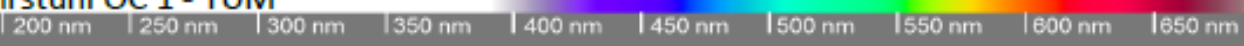

Datasheet FLT022

LZC-420

Basic Information

Type

Description

Manufacturer / Supplier

Order number / Date of purch.

Internal lot / serial number

Specification Manufacturer

Type / size

Mechanical specification

Electrical specification

Wavelength (range, typ.)

Spectral width (FWHM)

Datasheet

Fluorescent light tube

Luzchem LZC-420

n/a / Luzchem

n/a / 07/2017

2017-07 / FLT022

T5 tube, G5 socket

$16 \mathrm{~mm}$ diameter, $288 \mathrm{~mm}$ length

$8 \mathrm{~W}$

$400-440 \mathrm{~nm}$

$\sim 30 \mathrm{~nm}$

LES-420-016

Characterization

Description of measurement

Measured with Ocean-optics USB4000 spectrometer using a

calibrated setup (cosine corrector/fibre).

The cosine corrector was placed at $20 \mathrm{~mm}$ distance from a single fluorescent tube at half height.

Measured dominant wavelength / Int.

$421 \mathrm{~nm}$

$121 \mu \mathrm{W} / \mathrm{mm}^{2} \mathrm{~nm}$

Measured spectral width (FWHM)

$30 \mathrm{~nm}$

Integral Reference intensity / range

$4142 \mu \mathrm{W} / \mathrm{cm}^{2}$

$350-500 \mathrm{~nm}$

Spectrum

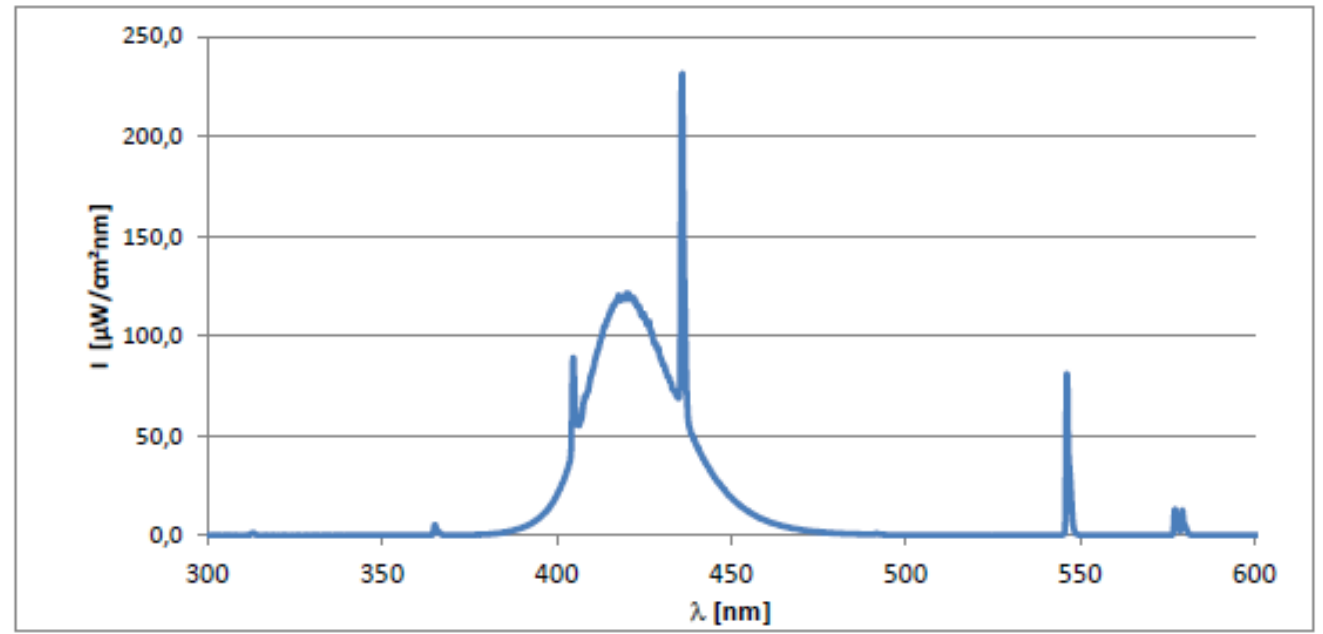




\section{NMR Spectra of All Compounds}

${ }^{1} \mathrm{H}(400 \mathrm{MHz})$ and ${ }^{13} \mathrm{C}\left\{{ }^{1} \mathrm{H}\right\} \mathrm{NMR}(101 \mathrm{MHz})$ spectra of compound $\mathbf{1 a}\left(\mathrm{CDCl}_{3}\right)$
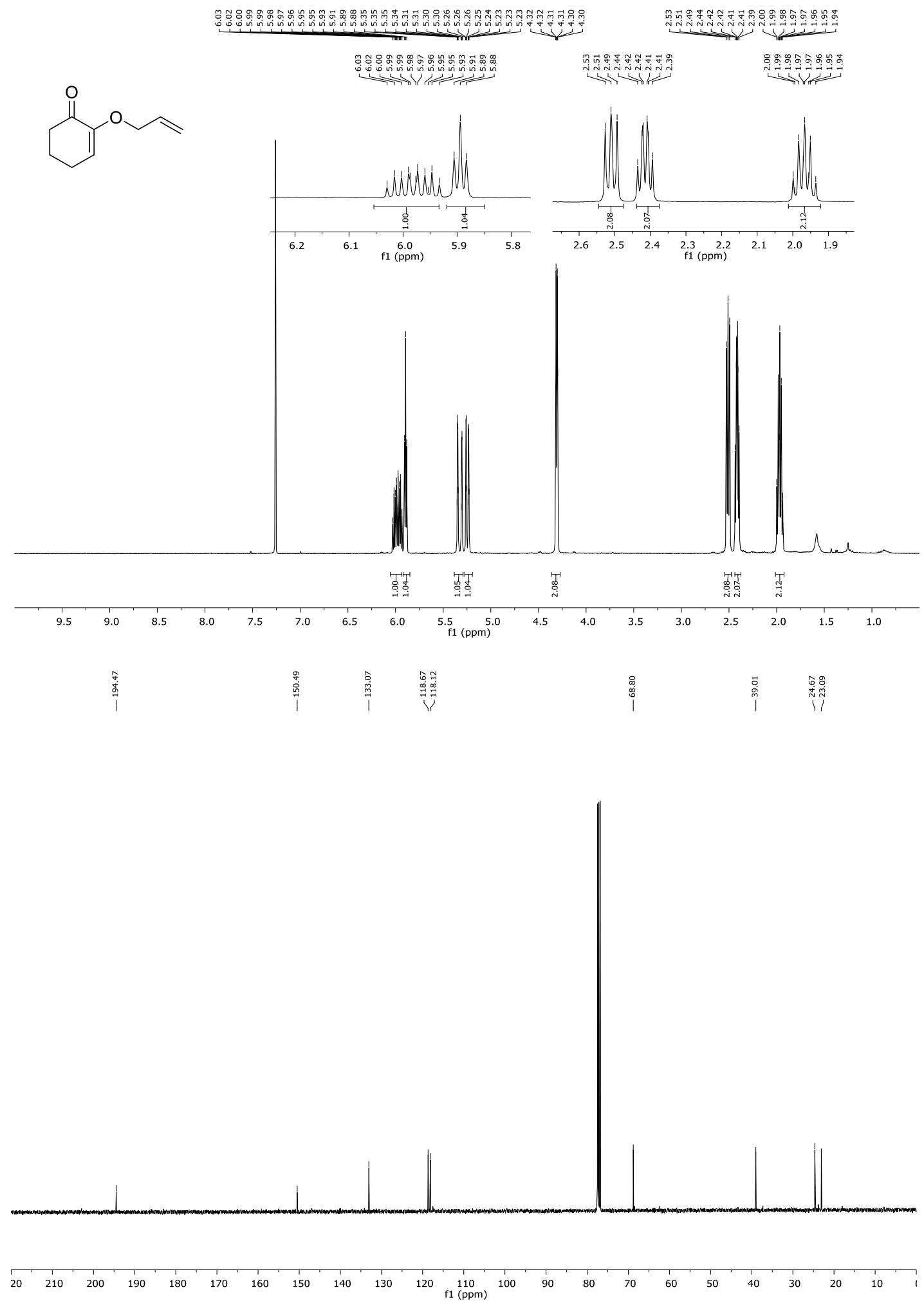
${ }^{1} \mathrm{H}(500 \mathrm{MHz})$ and ${ }^{13} \mathrm{C}\left\{{ }^{1} \mathrm{H}\right\} \mathrm{NMR}(101 \mathrm{MHz})$ spectra of compound $\mathbf{1 b}\left(\mathrm{CDCl}_{3}\right)$

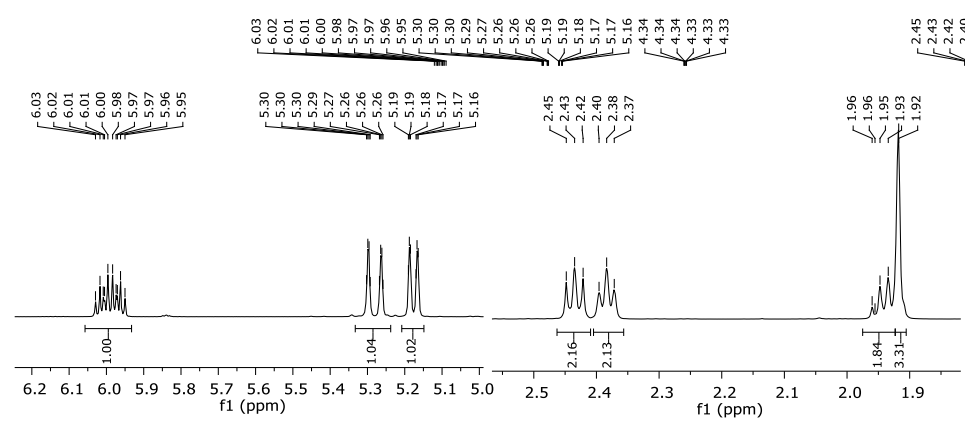<smiles>C=CCOC1=C(C)CCCC1=O</smiles>

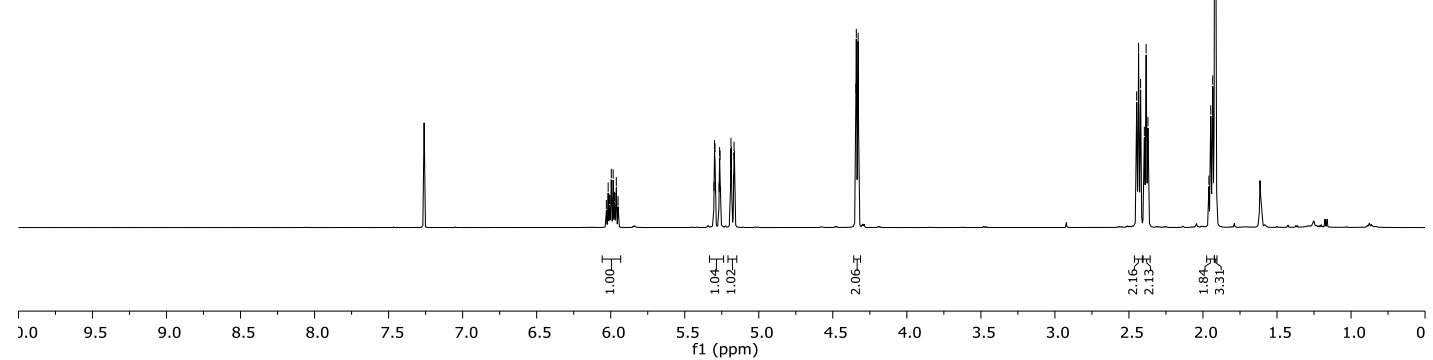

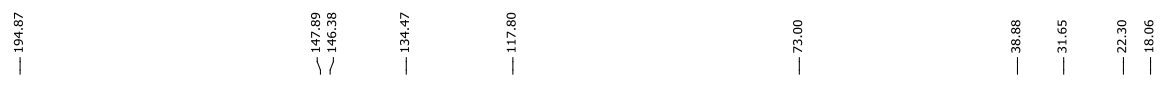

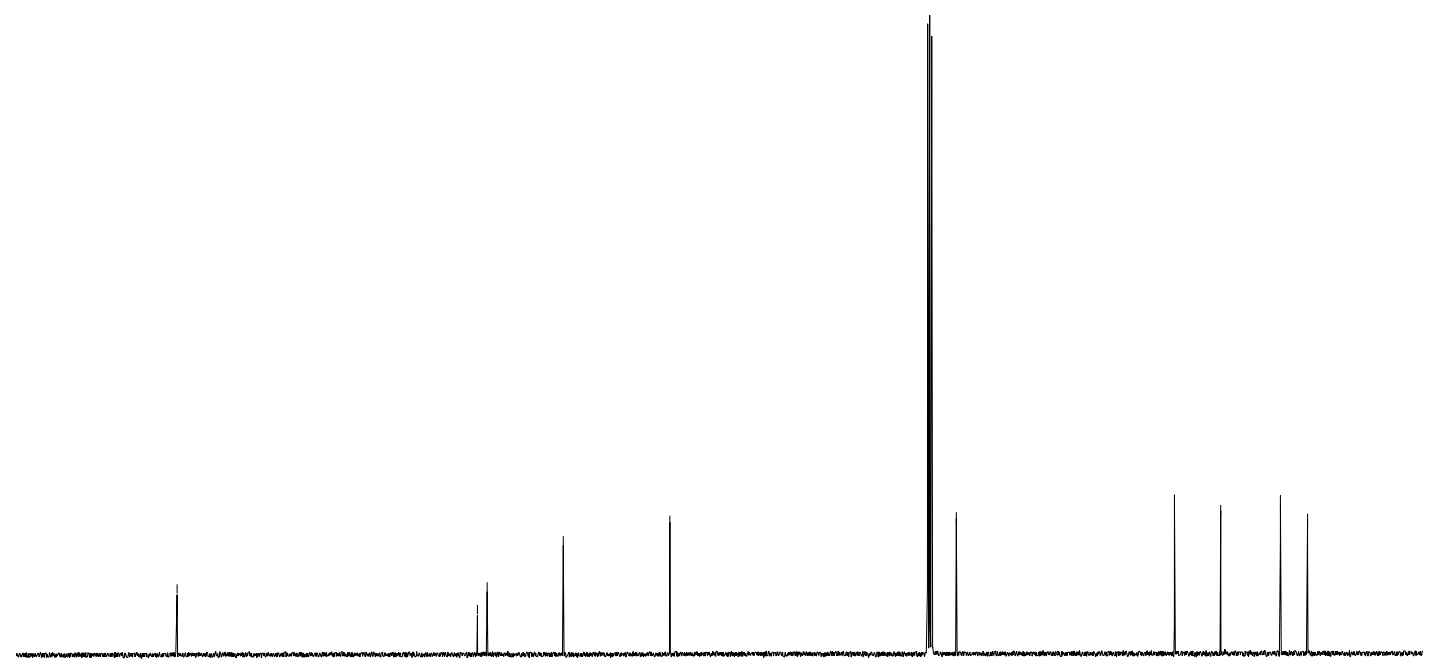

\begin{tabular}{llllllllllllllllllllllllll}
\hline 20 & 210 & 200 & 190 & 180 & 170 & 160 & 150 & 140 & 130 & 120 & 110 & 100 & 90 & 80 & 70 & 60 & 50 & 40 & 30 & 20 & 10 & 1
\end{tabular} 
${ }^{1} \mathrm{H}(500 \mathrm{MHz})$ and ${ }^{13} \mathrm{C}\left\{{ }^{1} \mathrm{H}\right\} \mathrm{NMR}(126 \mathrm{MHz})$ spectra of compound 1c $\left(\mathrm{CDCl}_{3}\right)$

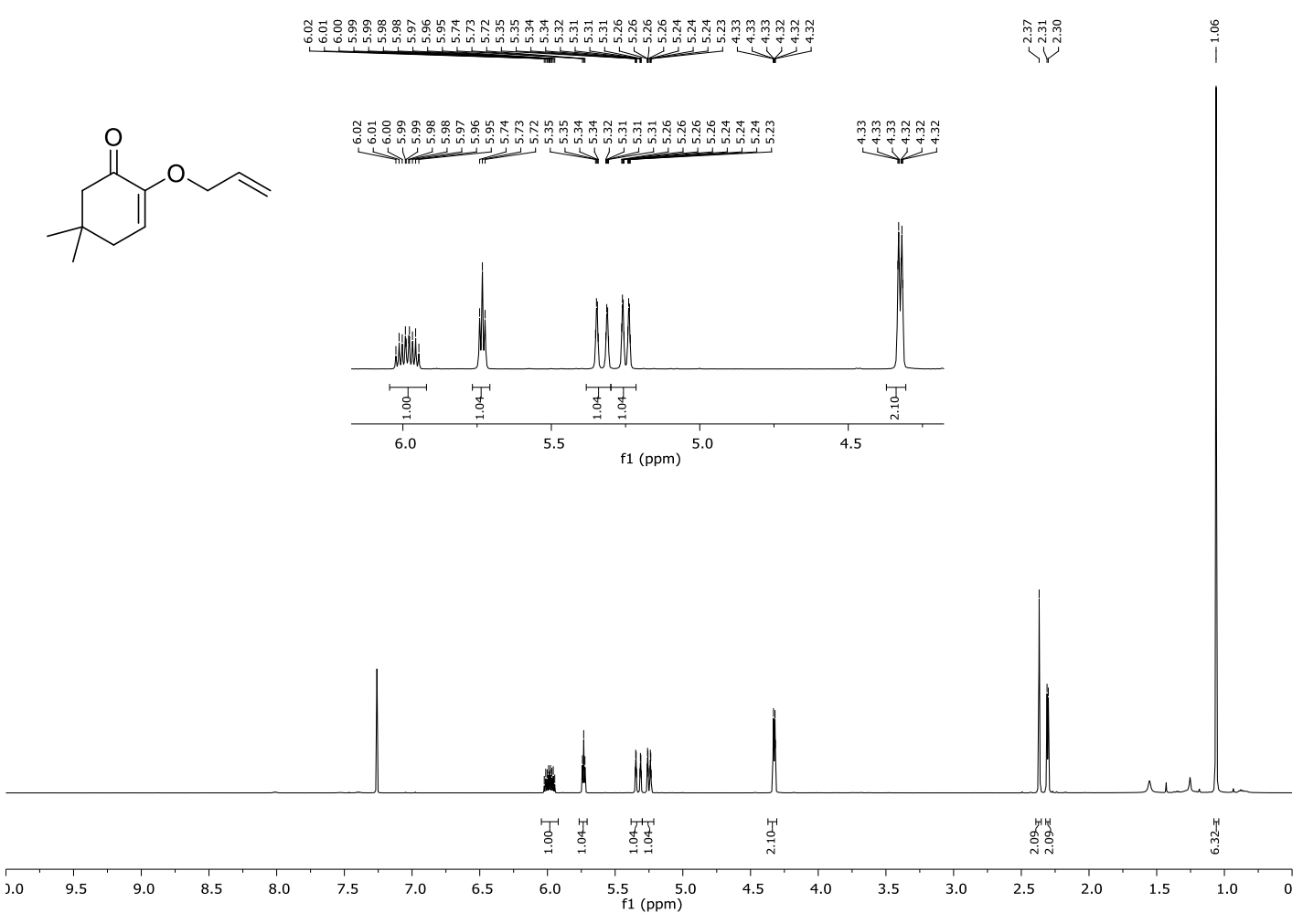

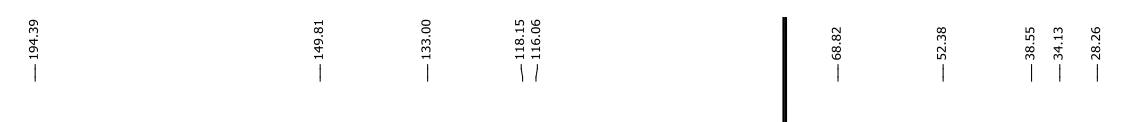

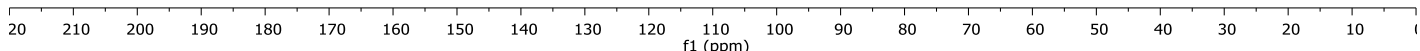


${ }^{1} \mathrm{H}(500 \mathrm{MHz})$ and ${ }^{13} \mathrm{C}\left\{{ }^{1} \mathrm{H}\right\} \mathrm{NMR}(126 \mathrm{MHz})$ spectra of compound $\mathbf{1 d}\left(\mathrm{CDCl}_{3}\right)$

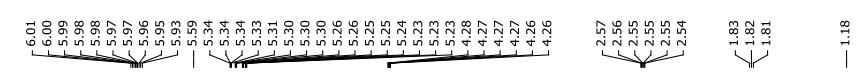<smiles>C=CCOC1=CC(C)(C)CCC1=O</smiles>
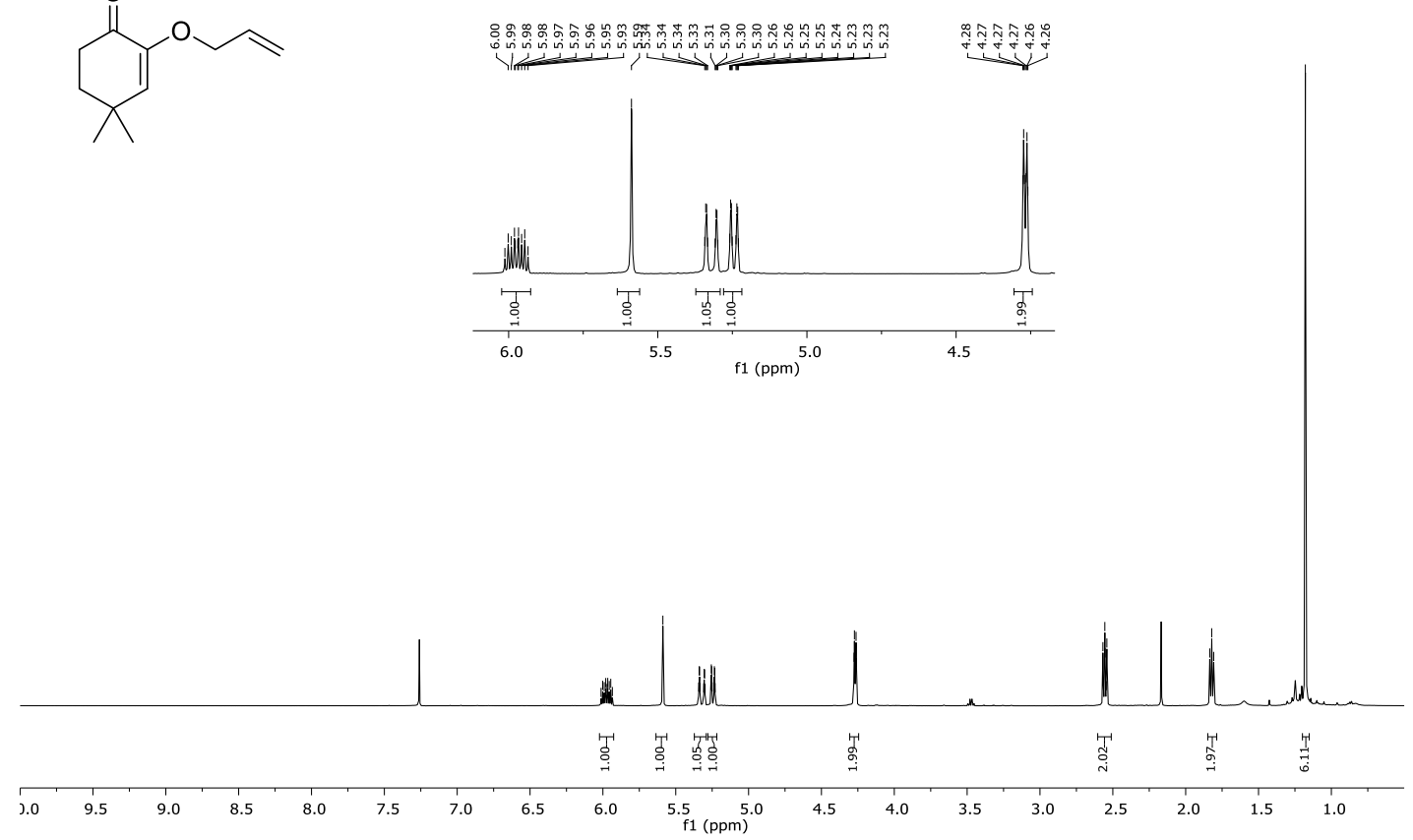

1

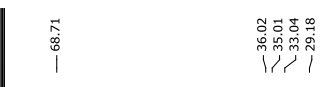

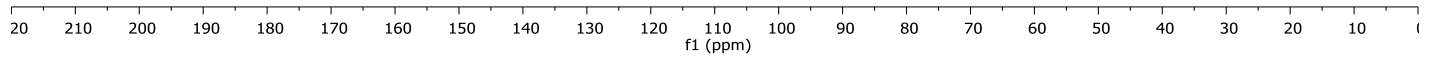


${ }^{1} \mathrm{H}(500 \mathrm{MHz})$ and ${ }^{13} \mathrm{C}\left\{{ }^{1} \mathrm{H}\right\} \mathrm{NMR}(126 \mathrm{MHz})$ spectra of compound 1e $\left(\mathrm{CDCl}_{3}\right)$

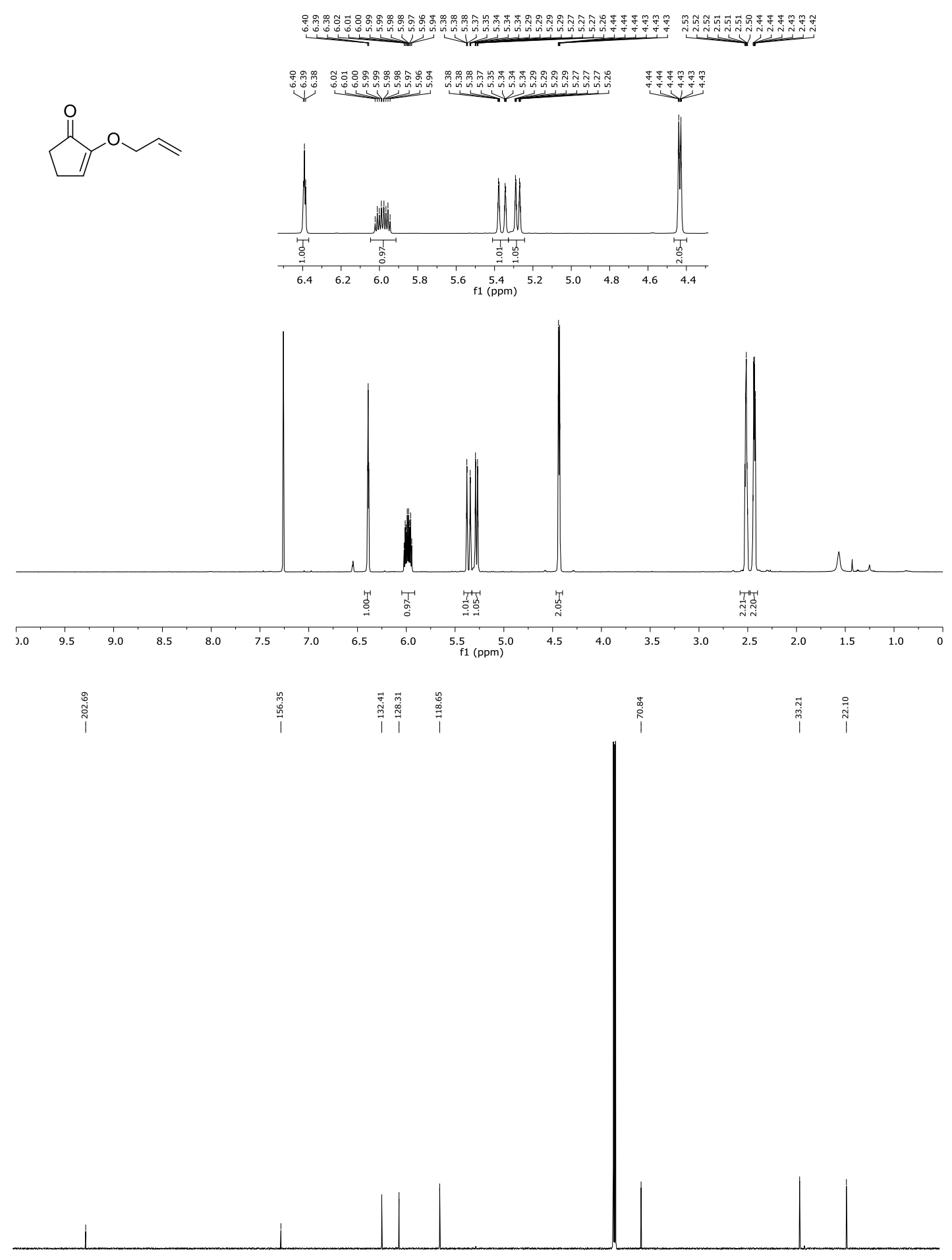

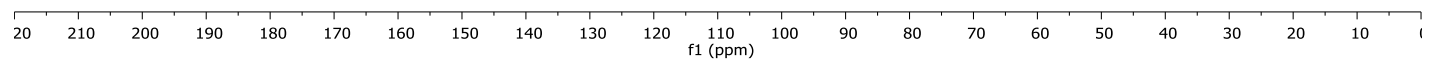


${ }^{1} \mathrm{H}(400 \mathrm{MHz})$ and ${ }^{13} \mathrm{C}\left\{{ }^{1} \mathrm{H}\right\}$ NMR (101 MHz) spectra of compound $\mathbf{1 f}\left(\mathrm{CDCl}_{3}\right)$

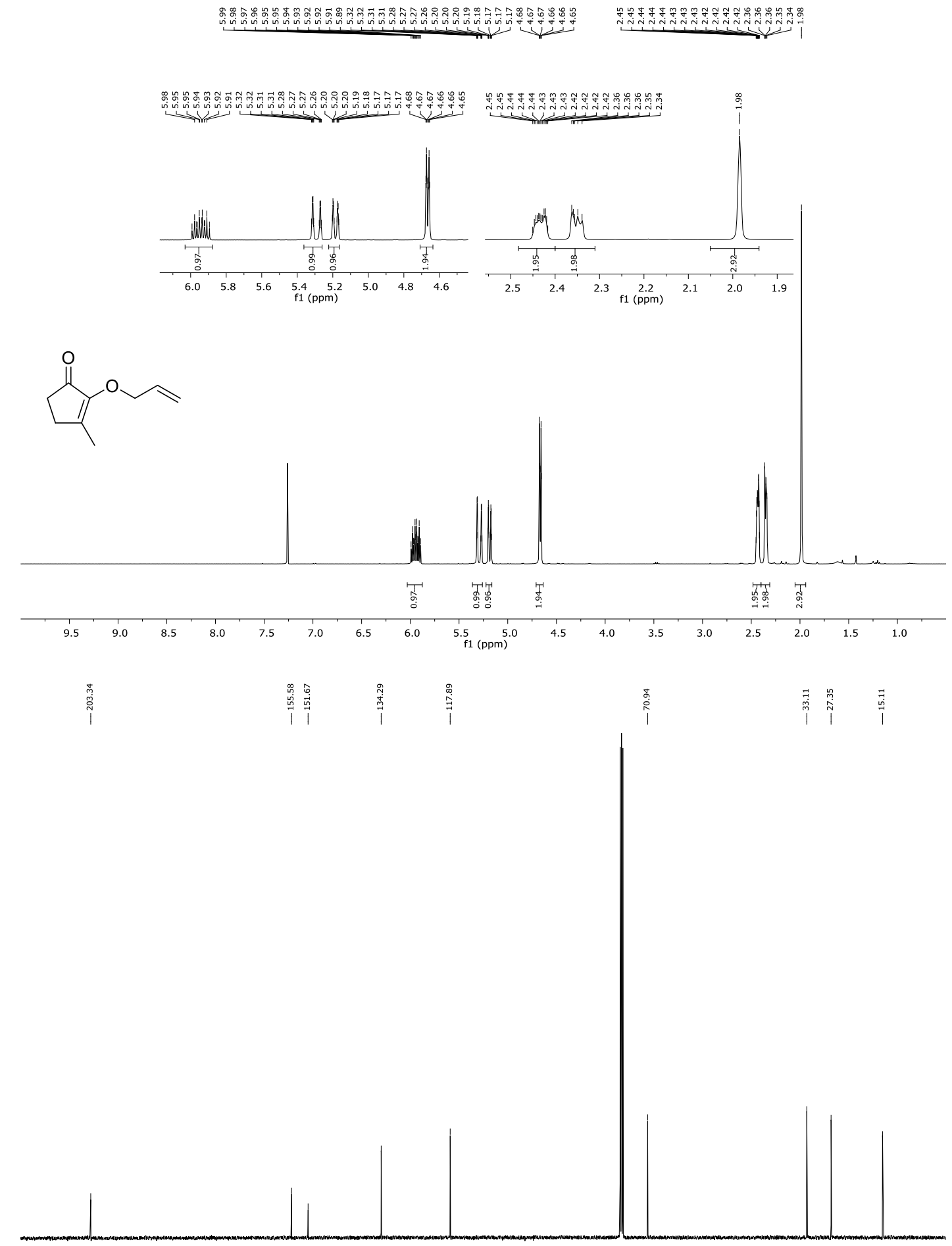

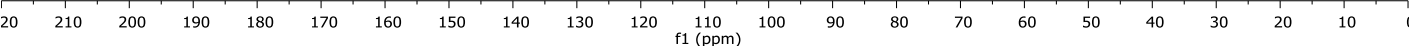


${ }^{1} \mathrm{H}(500 \mathrm{MHz})$ and ${ }^{13} \mathrm{C}\left\{{ }^{1} \mathrm{H}\right\}$ NMR $(75 \mathrm{MHz})$ spectra of compound 2a $\left(\mathrm{C}_{6} \mathrm{D}_{6}\right)$

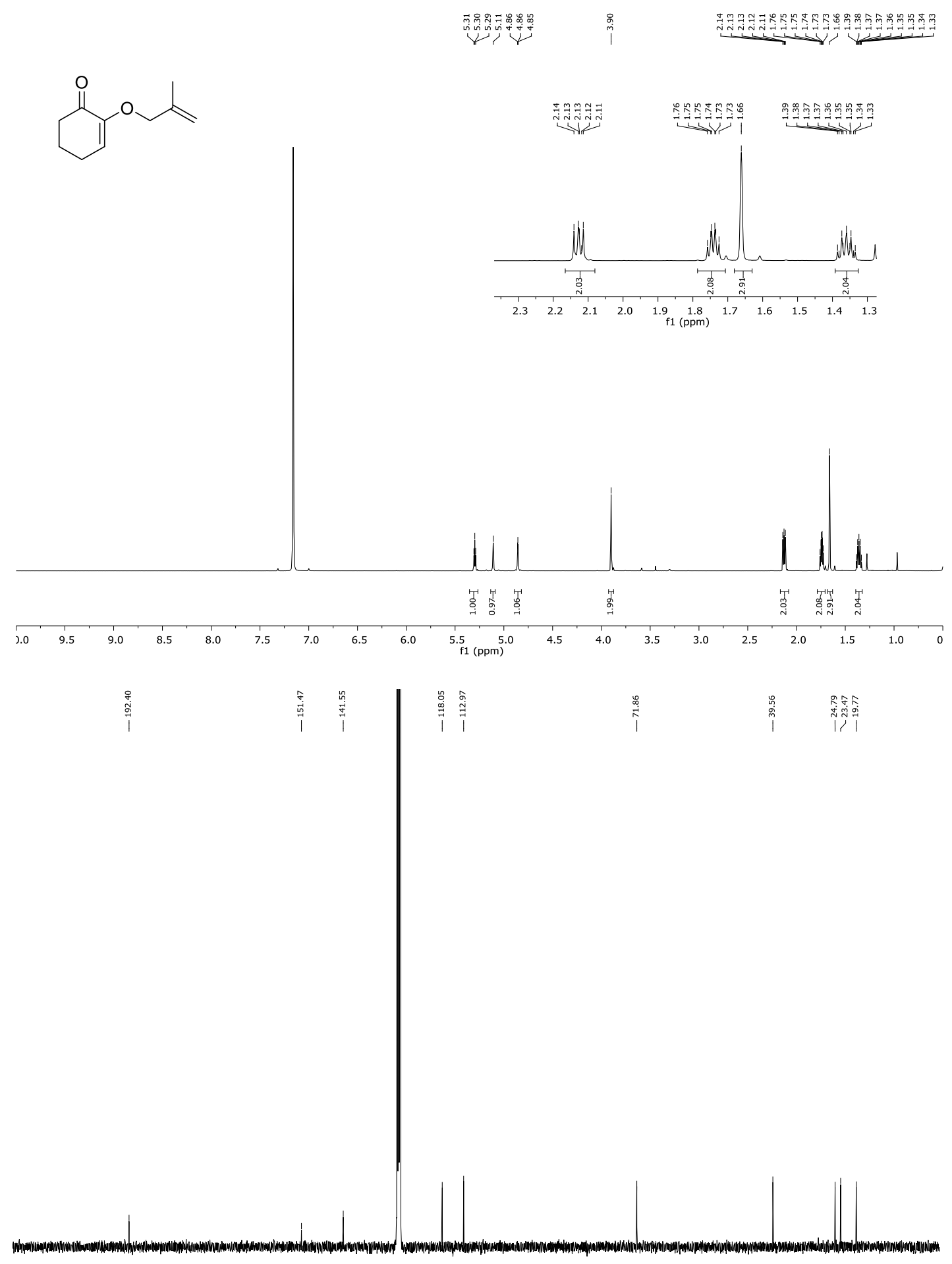

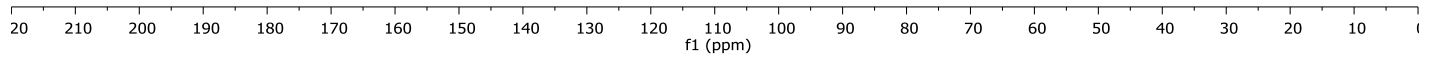


${ }^{1} \mathrm{H}(500 \mathrm{MHz})$ and ${ }^{13} \mathrm{C}\left\{{ }^{1} \mathrm{H}\right\} \mathrm{NMR}(126 \mathrm{MHz})$ spectra of compound $\mathbf{2 b}\left(\mathrm{CDCl}_{3}\right)$

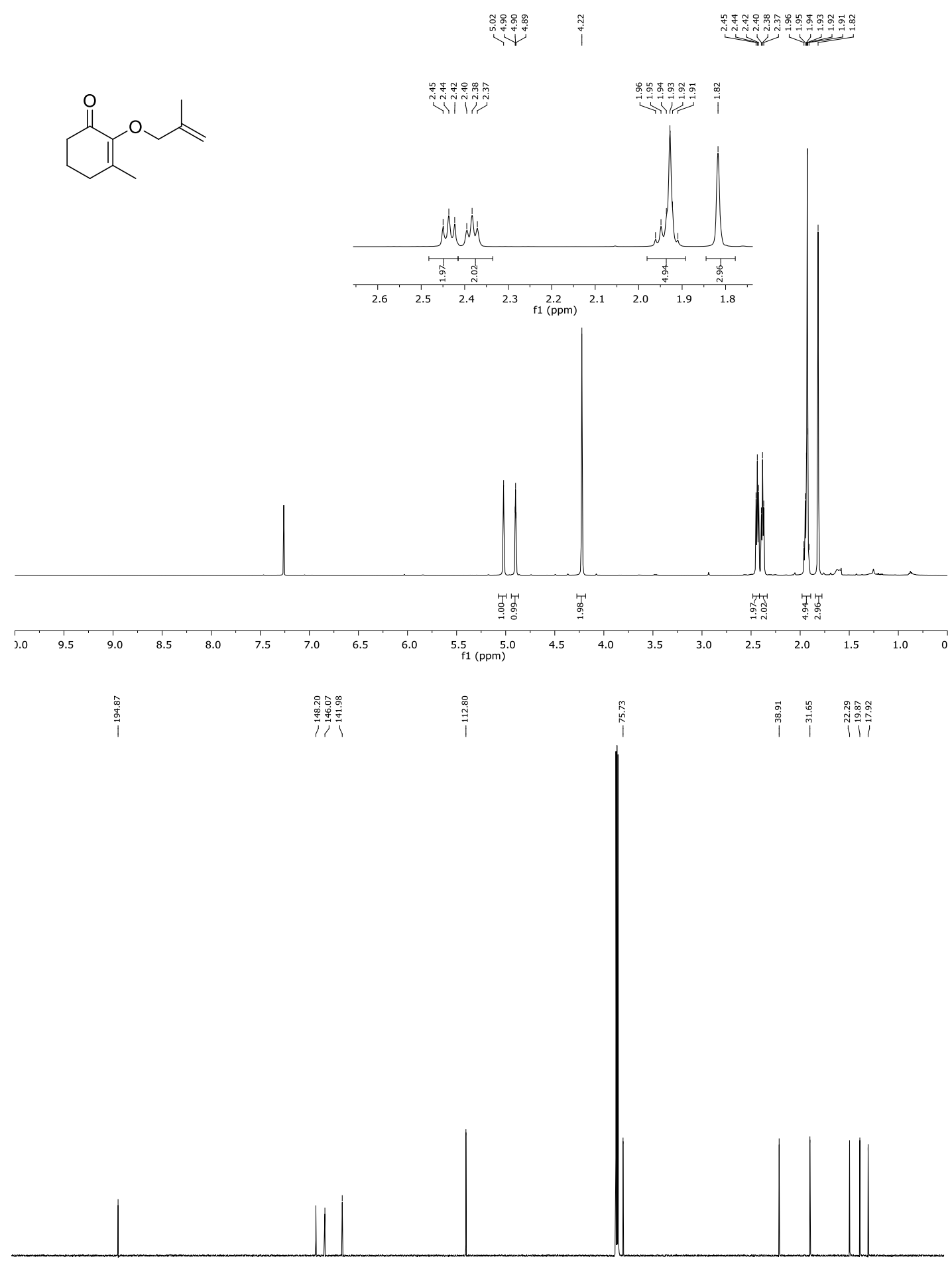

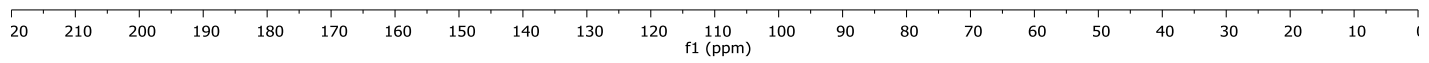


${ }^{1} \mathrm{H}(500 \mathrm{MHz})$ and ${ }^{13} \mathrm{C}\left\{{ }^{1} \mathrm{H}\right\}$ NMR (126 MHz) spectra of compound $2 \mathbf{c}\left(\mathrm{CDCl}_{3}\right)$

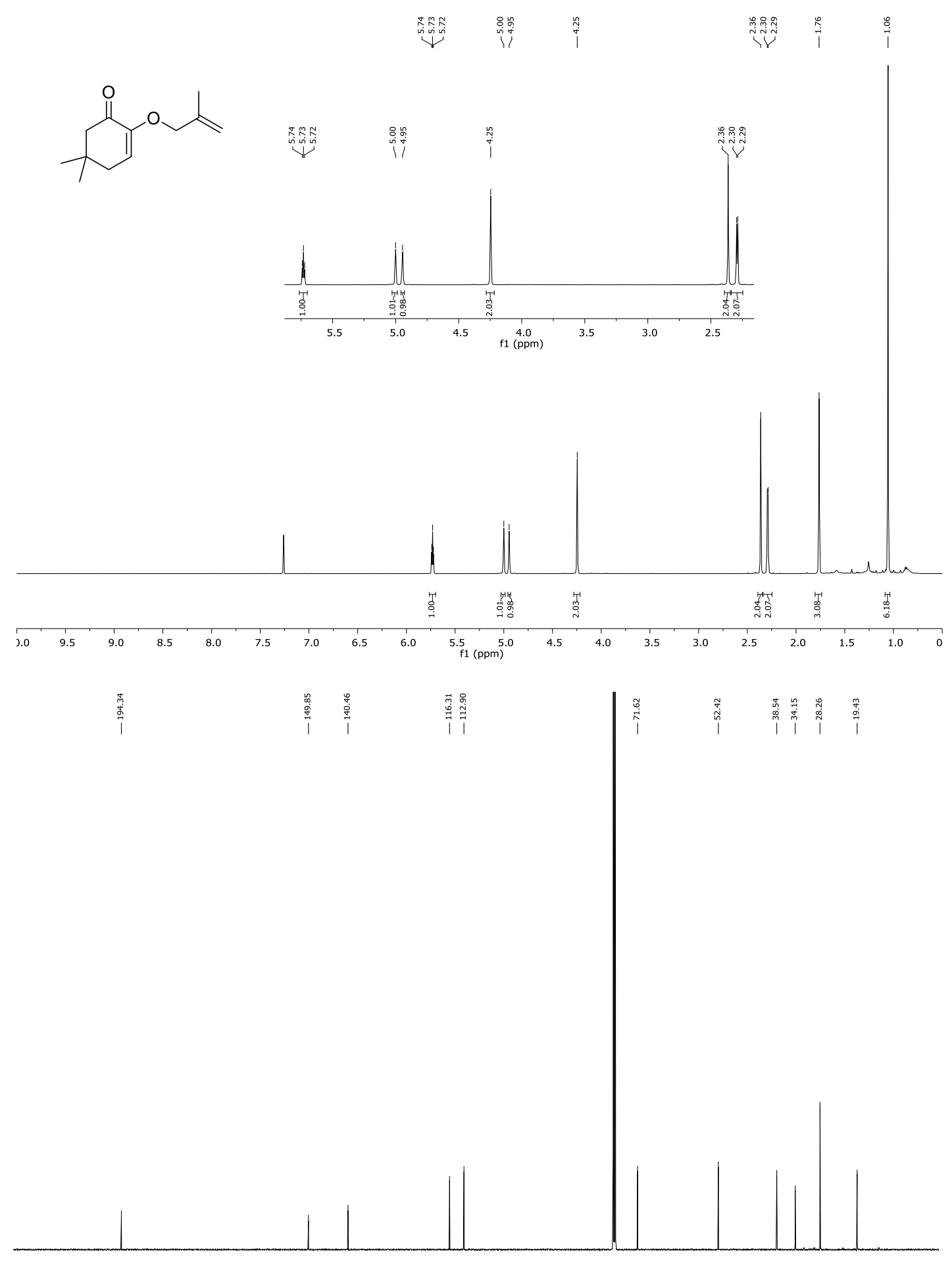

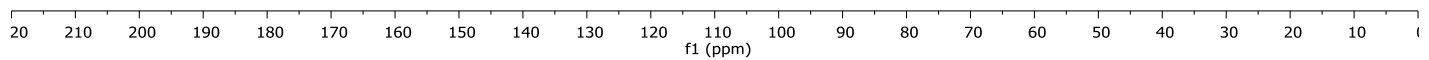


${ }^{1} \mathrm{H}(500 \mathrm{MHz})$ and ${ }^{13} \mathrm{C}\left\{{ }^{1} \mathrm{H}\right\} \mathrm{NMR}(126 \mathrm{MHz})$ spectra of compound $\mathbf{2 d}\left(\mathrm{CDCl}_{3}\right)$

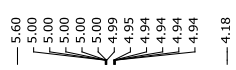<smiles>C=C(C)COC1=CC(C)(C)CCC1=O</smiles>
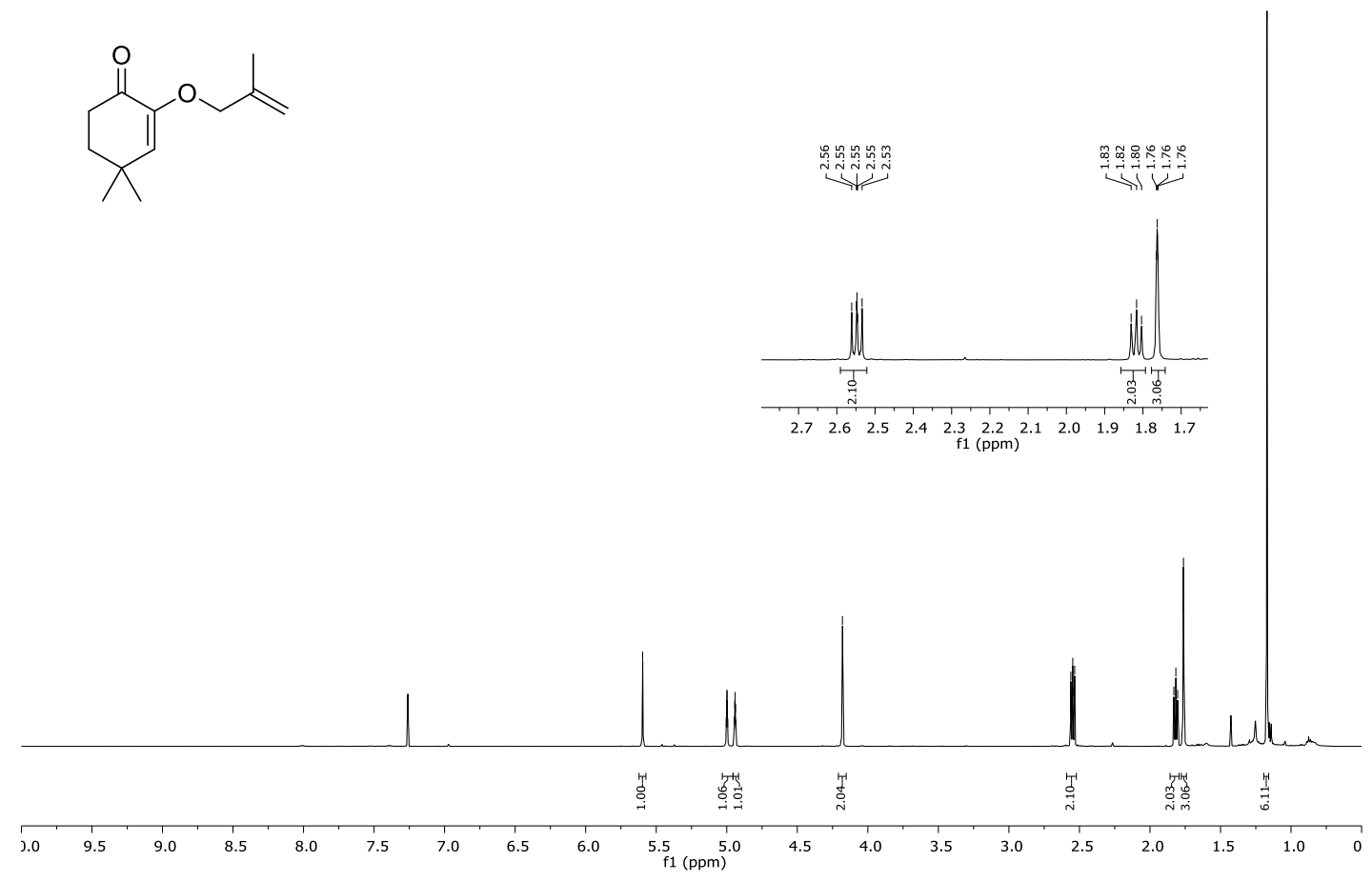

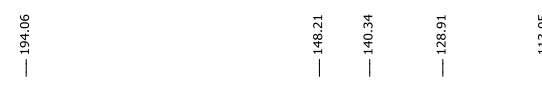

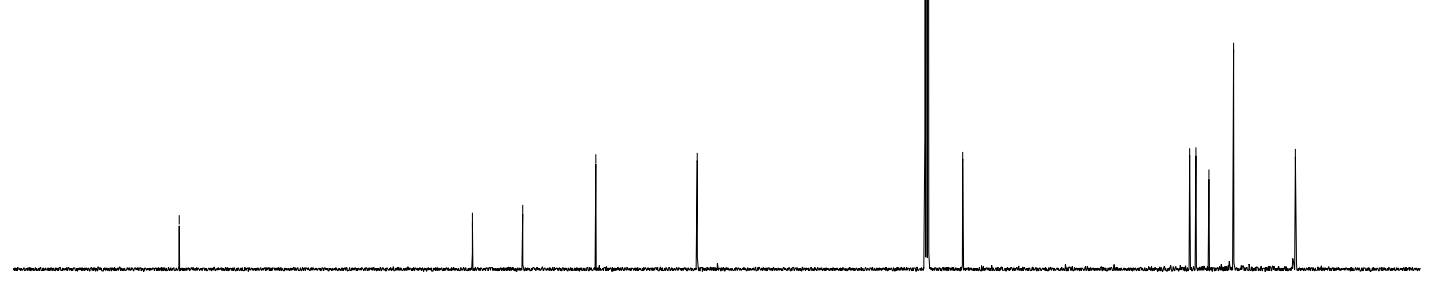

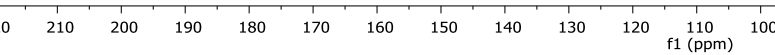


${ }^{1} \mathrm{H}(500 \mathrm{MHz})$ and ${ }^{13} \mathrm{C}\left\{{ }^{1} \mathrm{H}\right\}$ NMR (101 MHz) spectra of compound $2 \mathbf{e}\left(\mathrm{CDCl}_{3}\right)$

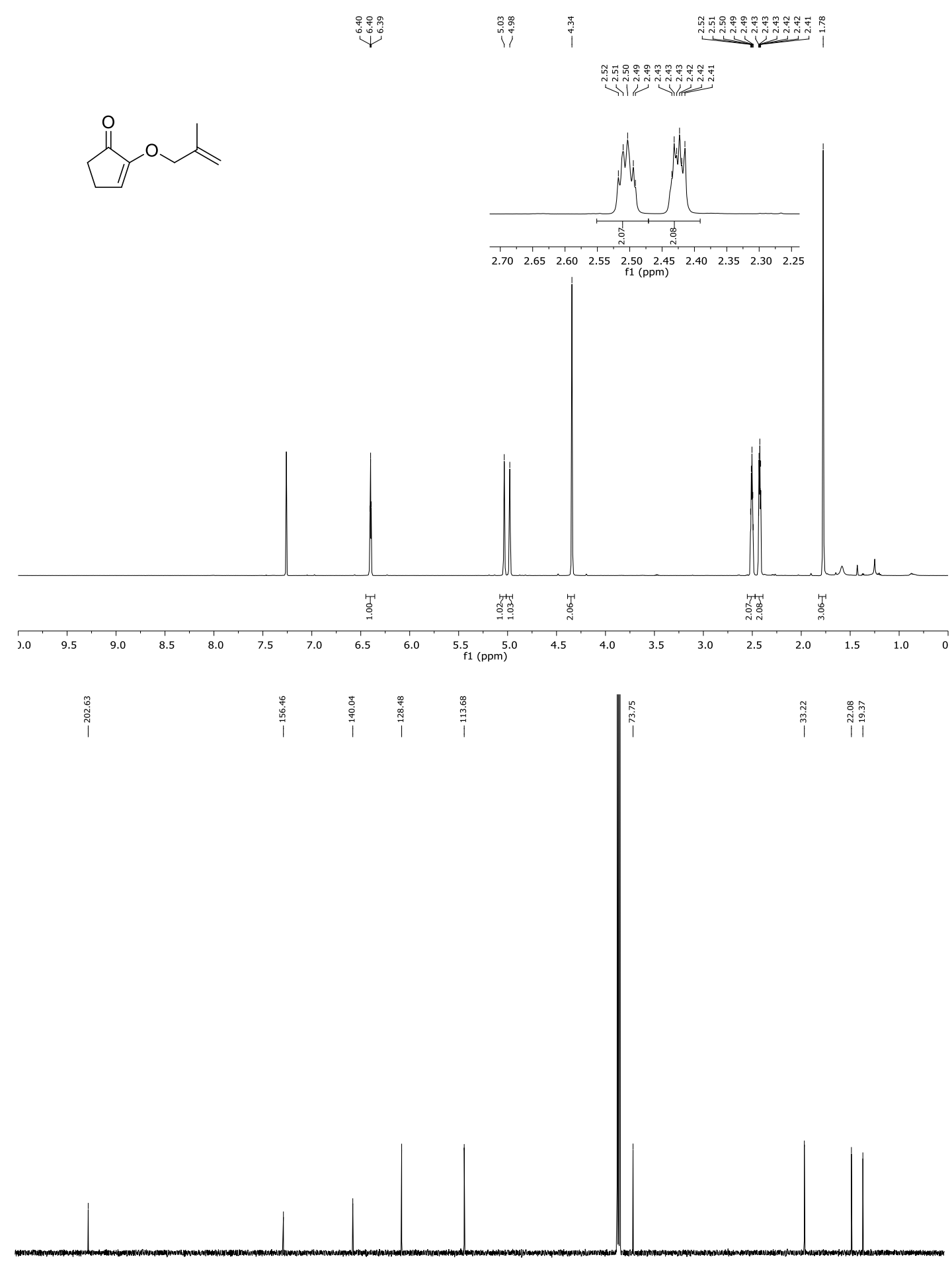

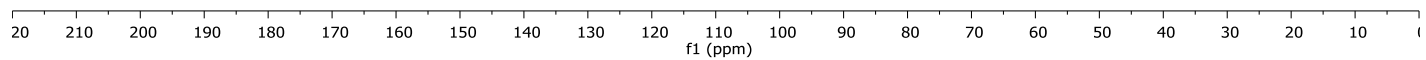


${ }^{1} \mathrm{H}(300 \mathrm{MHz})$ and ${ }^{13} \mathrm{C}\left\{{ }^{1} \mathrm{H}\right\} \mathrm{NMR}(75 \mathrm{MHz})$ spectra of compound $\mathbf{2 f}\left(\mathrm{C}_{6} \mathrm{D}_{6}\right)$

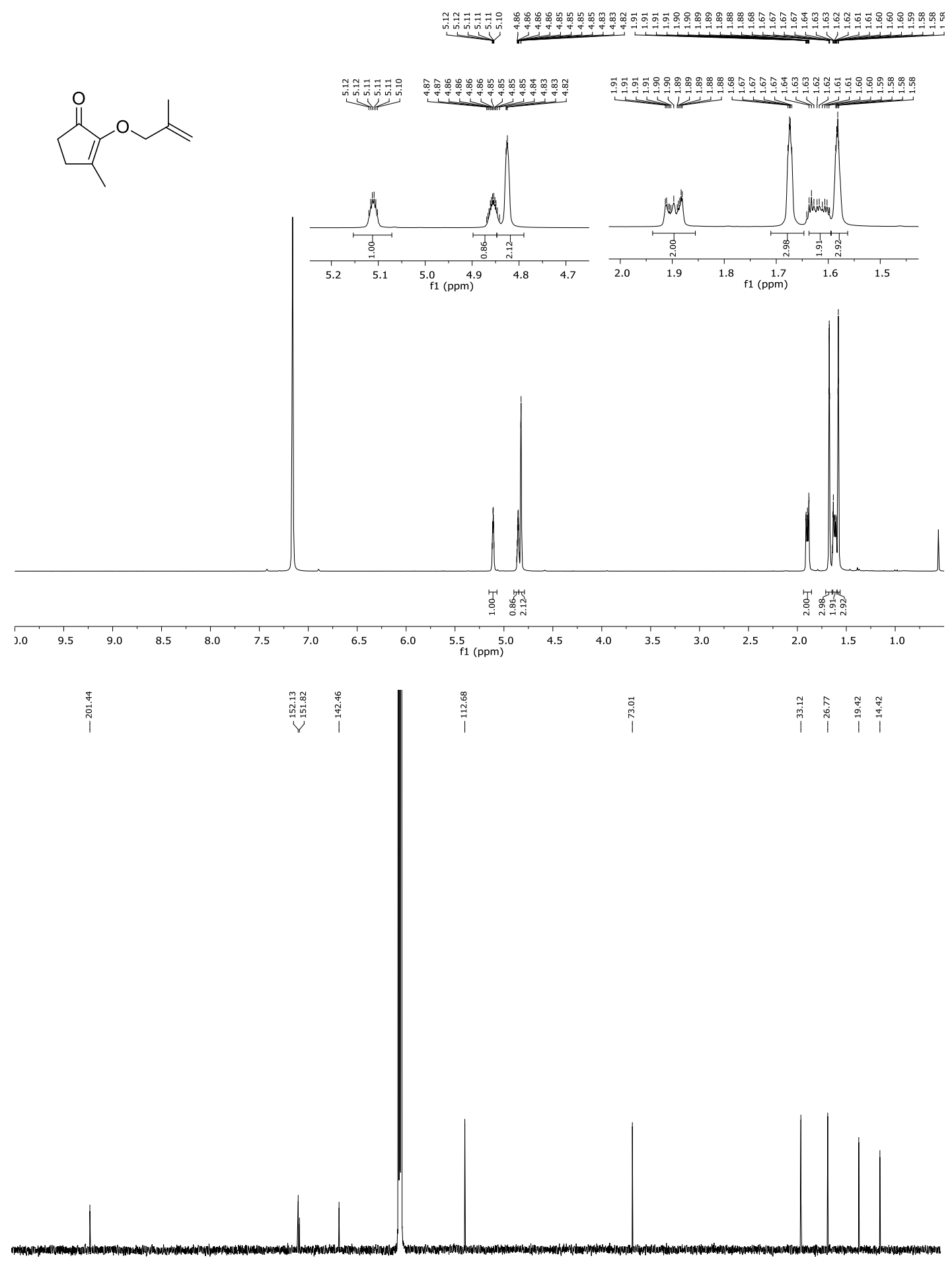

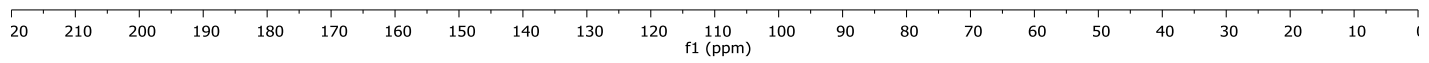


${ }^{1} \mathrm{H}(500 \mathrm{MHz})$ and ${ }^{13} \mathrm{C}\left\{{ }^{1} \mathrm{H}\right\} \mathrm{NMR}(101 \mathrm{MHz})$ spectra of compound 3a $\left(\mathrm{CDCl}_{3}\right)$
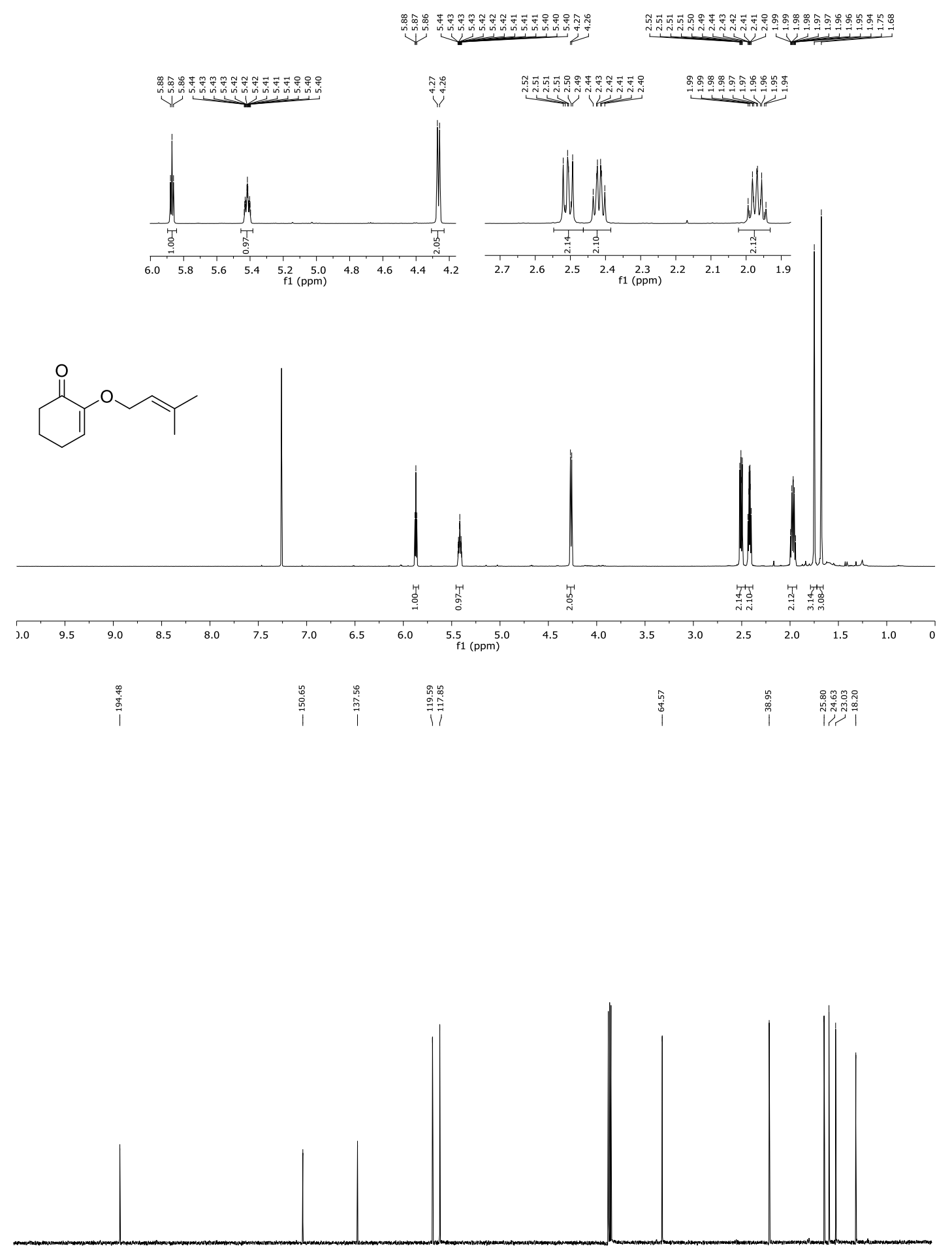

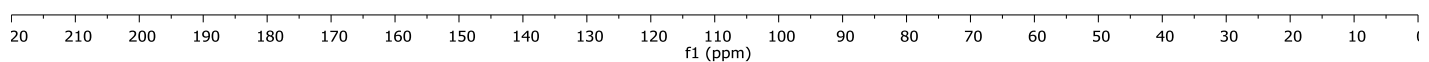


${ }^{1} \mathrm{H}(500 \mathrm{MHz})$ and ${ }^{13} \mathrm{C}\left\{{ }^{1} \mathrm{H}\right\} \mathrm{NMR}(126 \mathrm{MHz})$ spectra of compound $\mathbf{3 b}\left(\mathrm{CDCl}_{3}\right)$

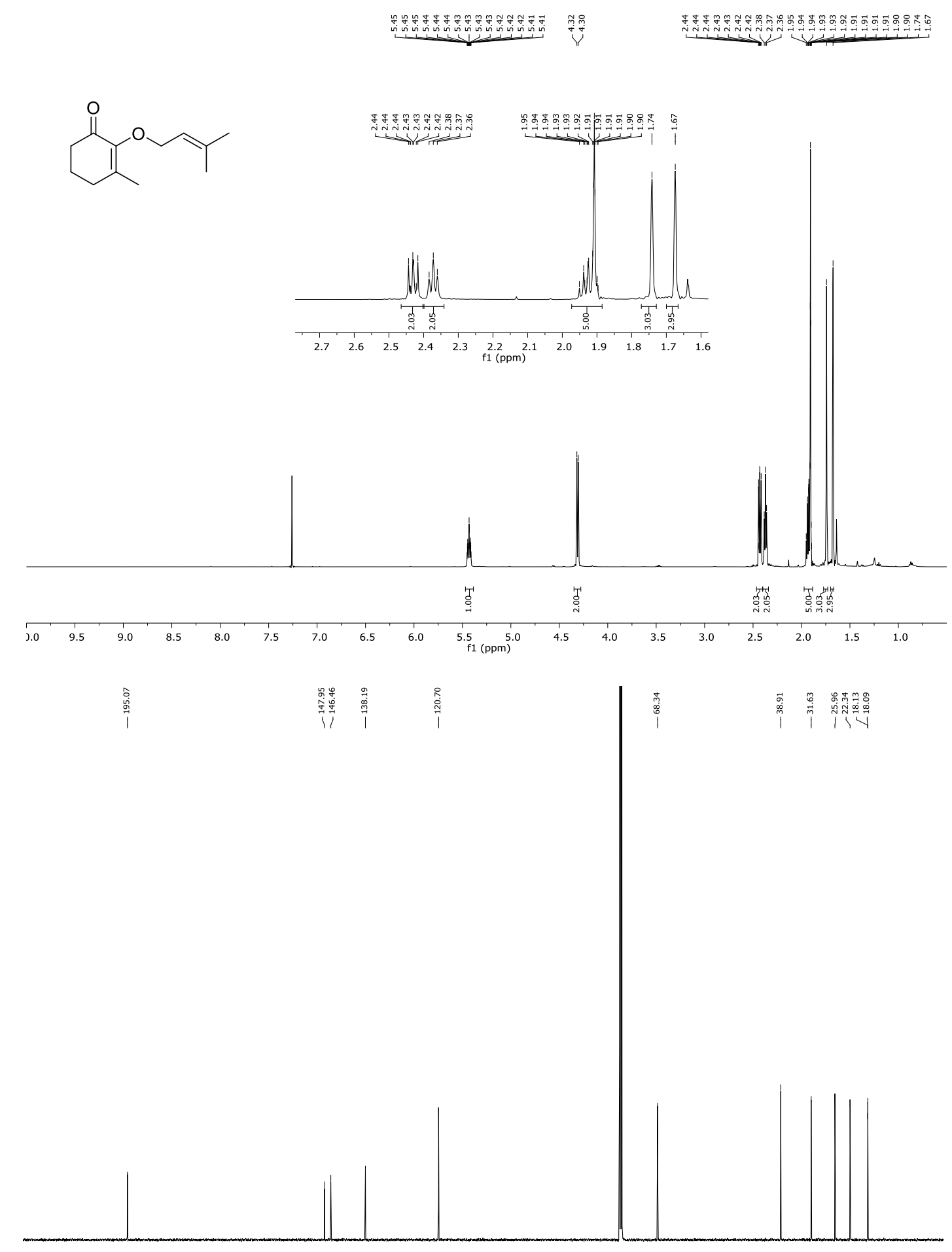

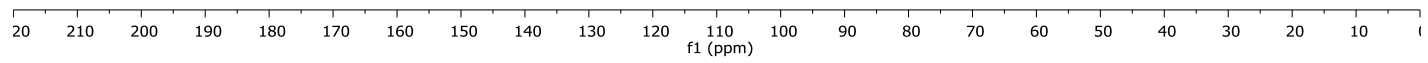


${ }^{1} \mathrm{H}(500 \mathrm{MHz})$ and ${ }^{13} \mathrm{C}\left\{{ }^{1} \mathrm{H}\right\} \mathrm{NMR}(126 \mathrm{MHz})$ spectra of compound $3 \mathrm{c}\left(\mathrm{CDCl}_{3}\right)$ بัy<smiles>CC(C)=CCOC1=CCC(C)(C)CC1=O</smiles>
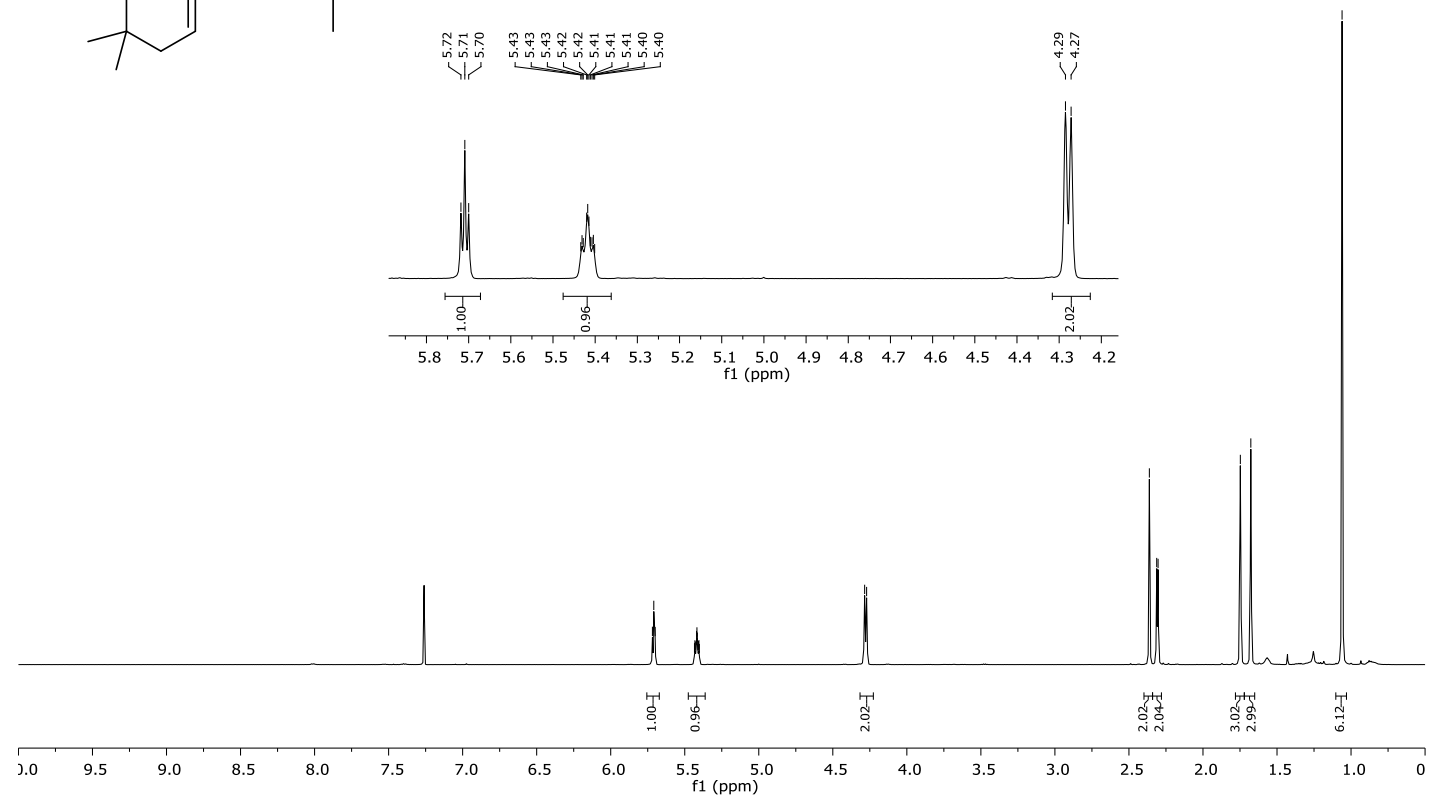

章

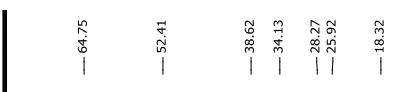

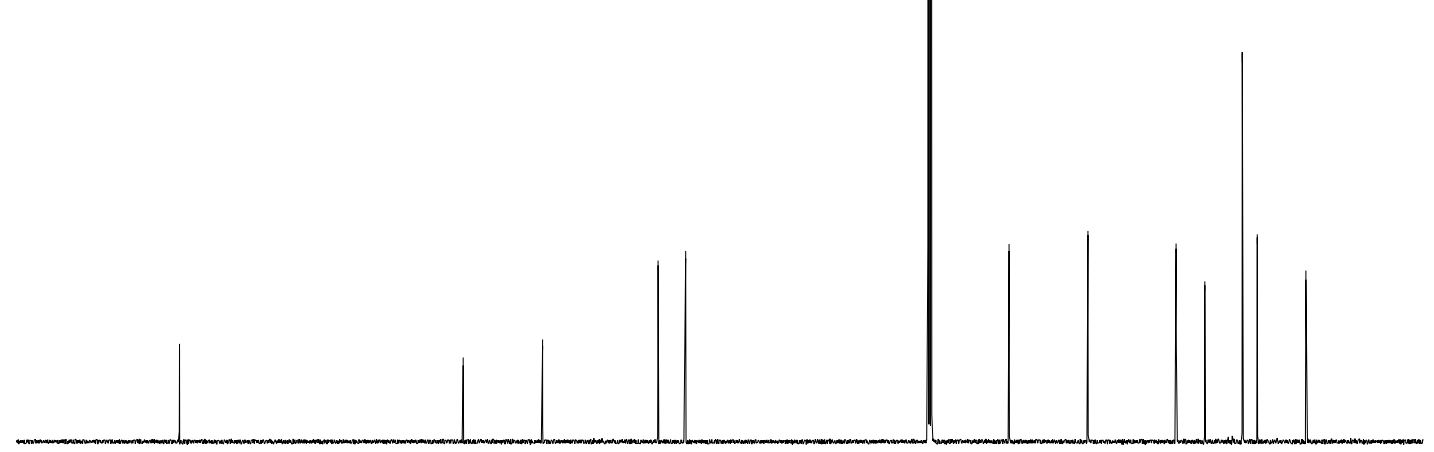

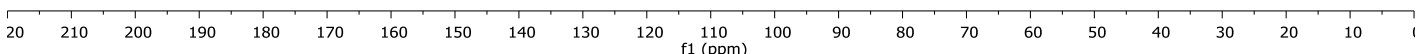


${ }^{1} \mathrm{H}(500 \mathrm{MHz})$ and ${ }^{13} \mathrm{C}\left\{{ }^{1} \mathrm{H}\right\} \mathrm{NMR}(126 \mathrm{MHz})$ spectra of compound $\mathbf{3 e}\left(\mathrm{CDCl}_{3}\right)$

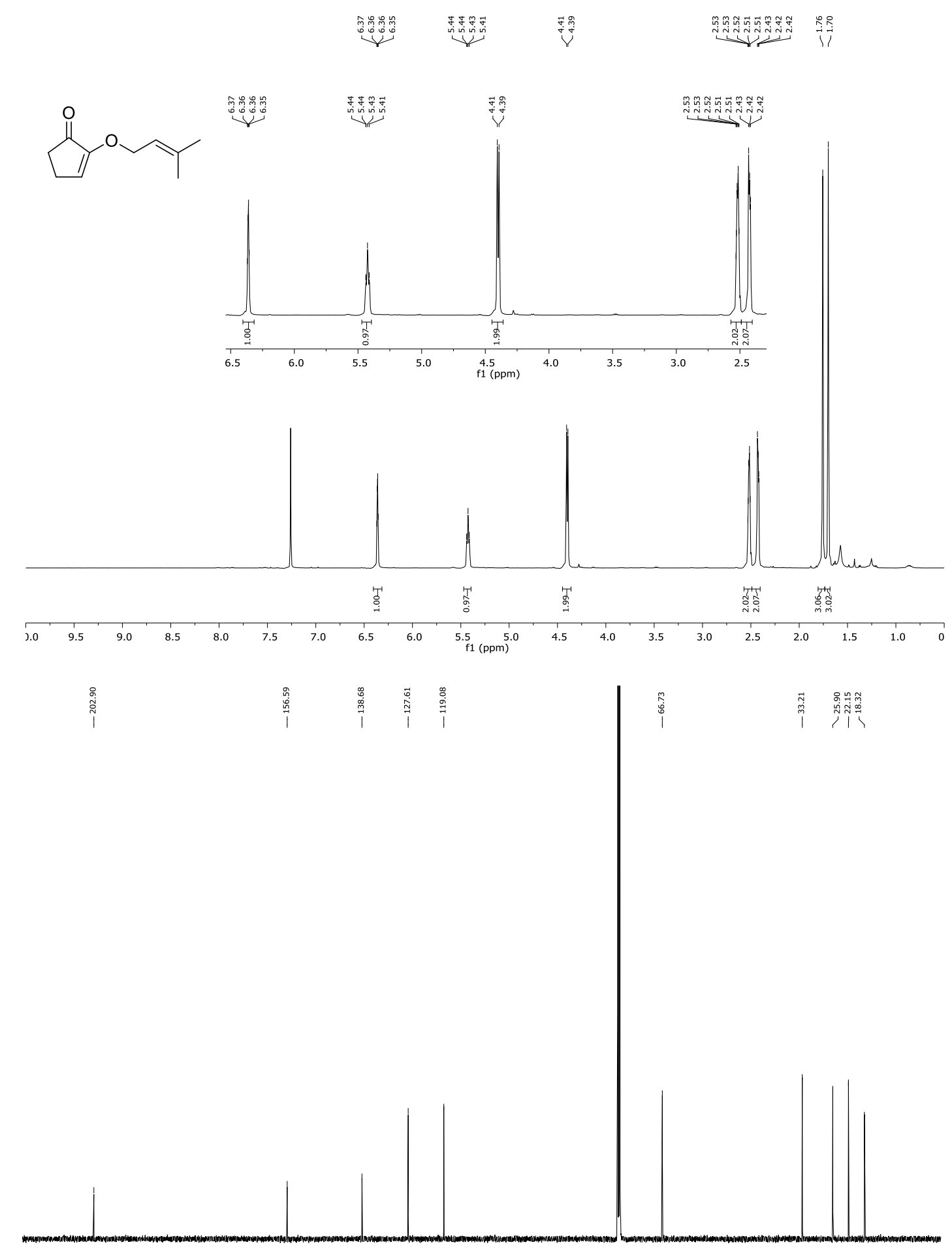

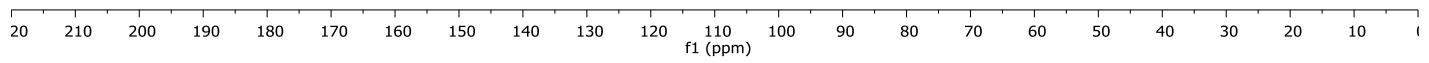


${ }^{1} \mathrm{H}(300 \mathrm{MHz})$ and ${ }^{13} \mathrm{C}\left\{{ }^{1} \mathrm{H}\right\}$ NMR (75 MHz) spectra of compound $\mathbf{3} \mathbf{f}\left(\mathrm{C}_{6} \mathrm{D}_{6}\right)$

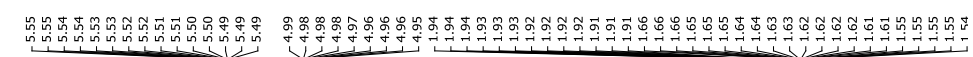
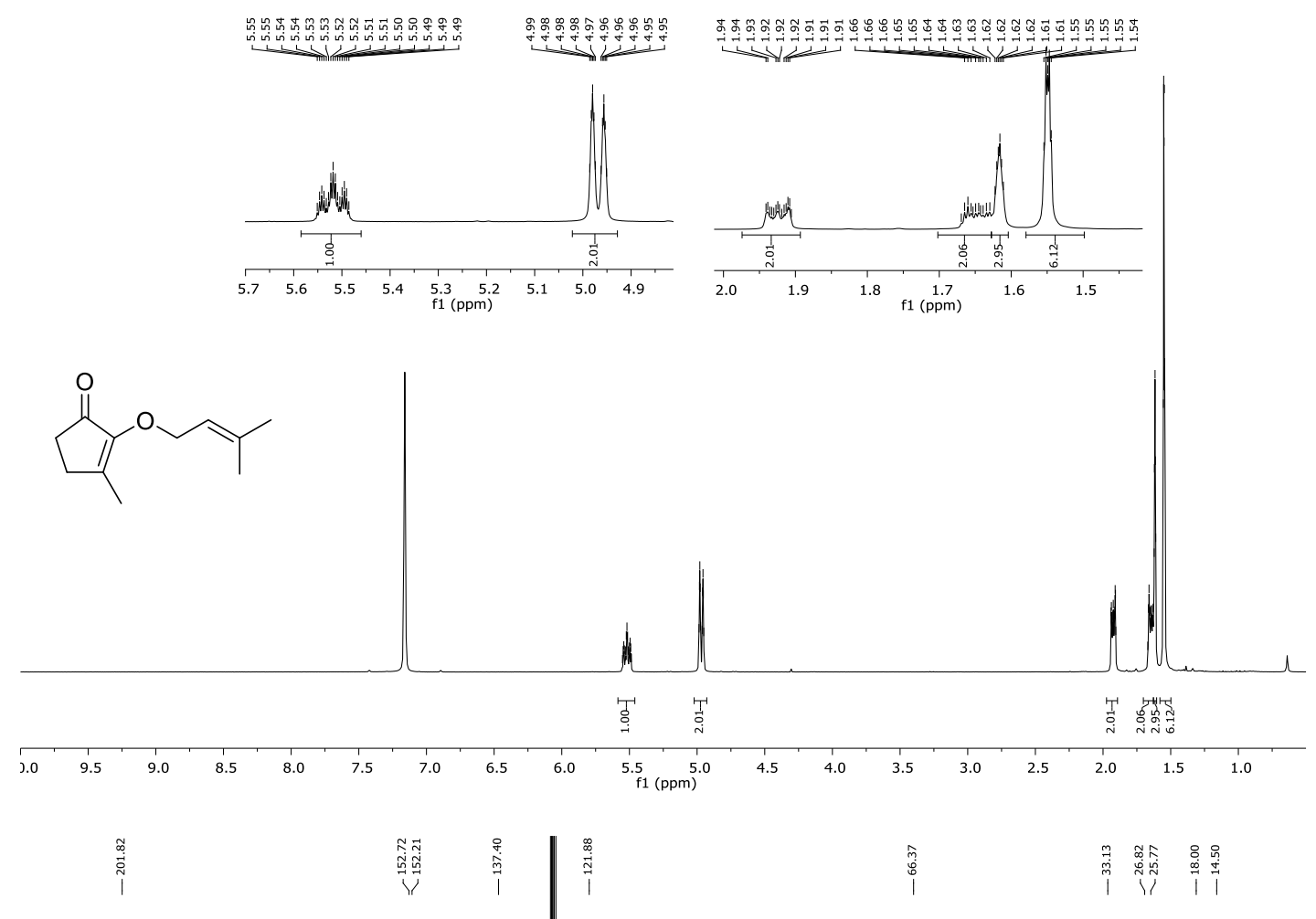

$\hat{m}$
0
0
0
1

|

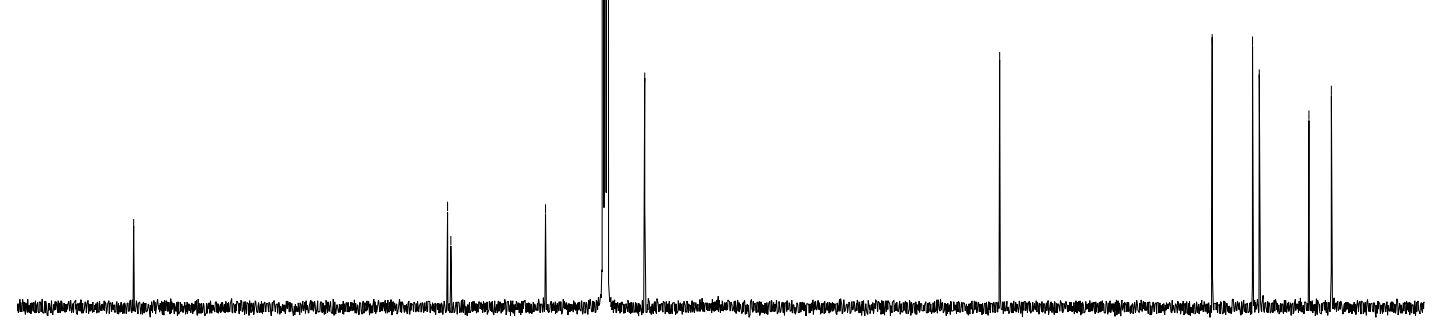

20

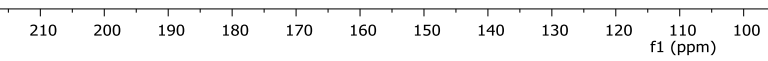


${ }^{1} \mathrm{H}(300 \mathrm{MHz})$ and ${ }^{13} \mathrm{C}\left\{{ }^{1} \mathrm{H}\right\} \mathrm{NMR}(75 \mathrm{MHz})$ spectra of 3-Ethoxy-5,5-dimethylcyclohex-2-en-1one $\left(\mathrm{CDCl}_{3}\right)$
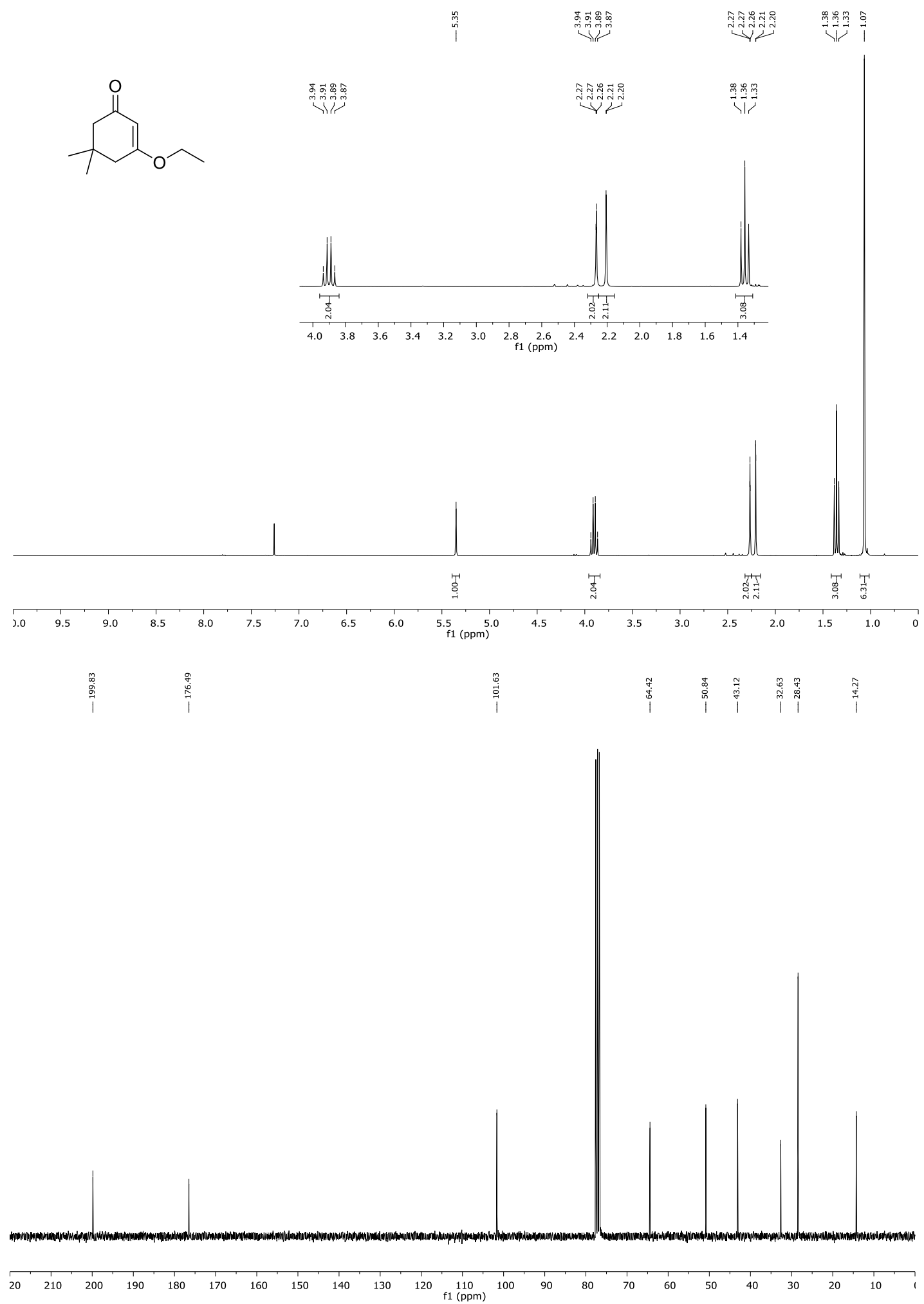
${ }^{1} \mathrm{H}(400 \mathrm{MHz})$ and ${ }^{13} \mathrm{C}\left\{{ }^{1} \mathrm{H}\right\}$ NMR (101 MHz) spectra of 5,5-Dimethylcyclohex-2-en-1-one $\left(\mathrm{CDCl}_{3}\right)$

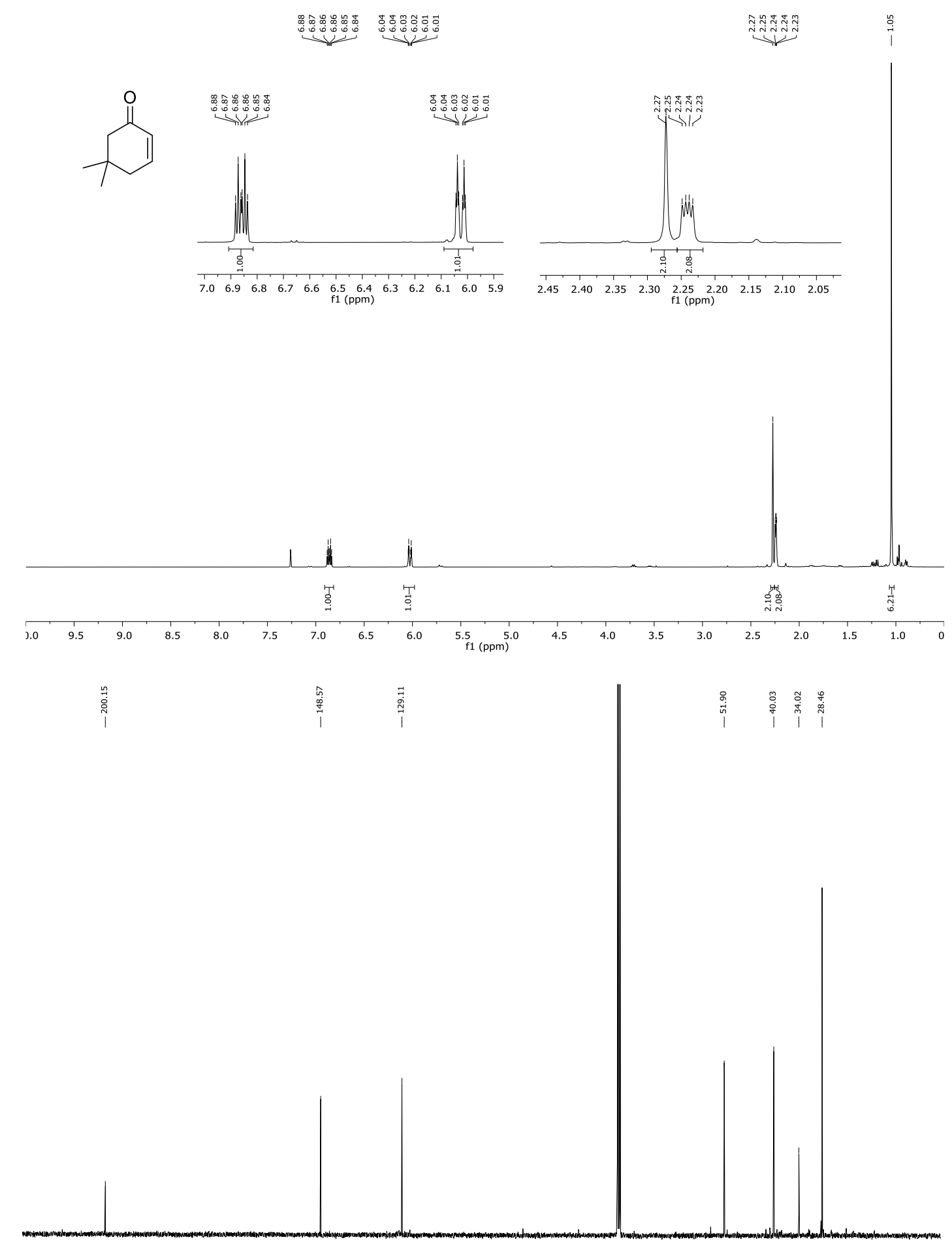

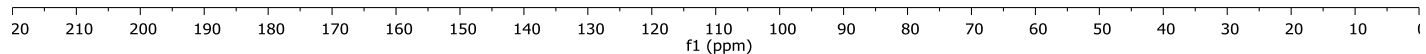


${ }^{1} \mathrm{H}(400 \mathrm{MHz})$ and ${ }^{13} \mathrm{C}\left\{{ }^{1} \mathrm{H}\right\} \mathrm{NMR}(101 \mathrm{MHz})$ spectra of compound $4 \mathbf{c}\left(\mathrm{CDCl}_{3}\right)$<smiles>CC1(C)CC(=O)C2OC2C1</smiles>
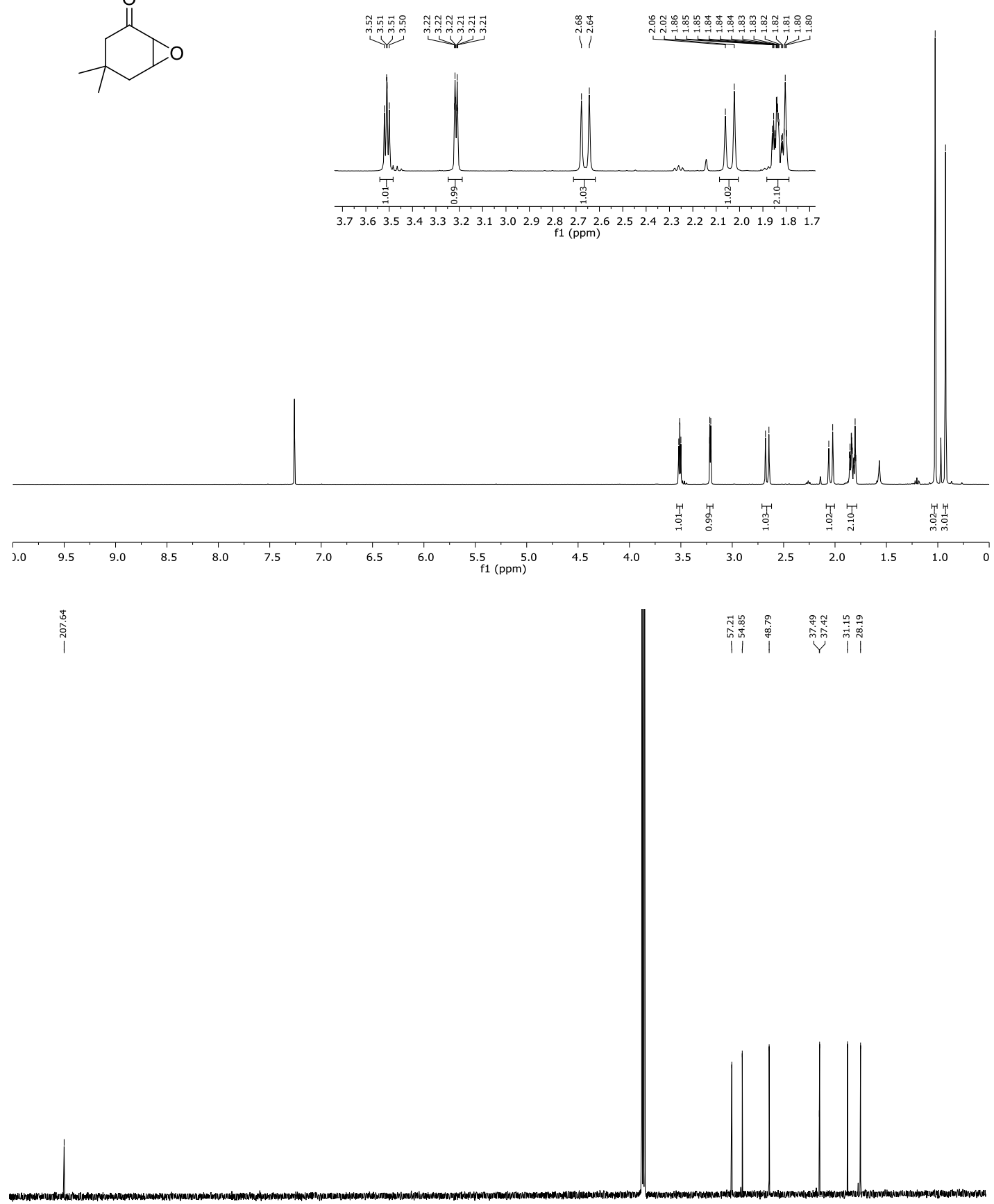

$\begin{array}{llllllllllllllllllllllllllll} & 20 & 210 & 200 & 190 & 180 & 170 & 160 & 150 & 140 & 130 & 120 & 110 & 100 & 90 & 80 & 70 & 60 & 50 & 40 & 30 & 20 & 10 & \end{array}$ 
${ }^{1} \mathrm{H}(500 \mathrm{MHz})$ and ${ }^{13} \mathrm{C}\left\{{ }^{1} \mathrm{H}\right\} \mathrm{NMR}(126 \mathrm{MHz})$ spectra of compound $\mathbf{4 d}\left(\mathrm{CDCl}_{3}\right)$

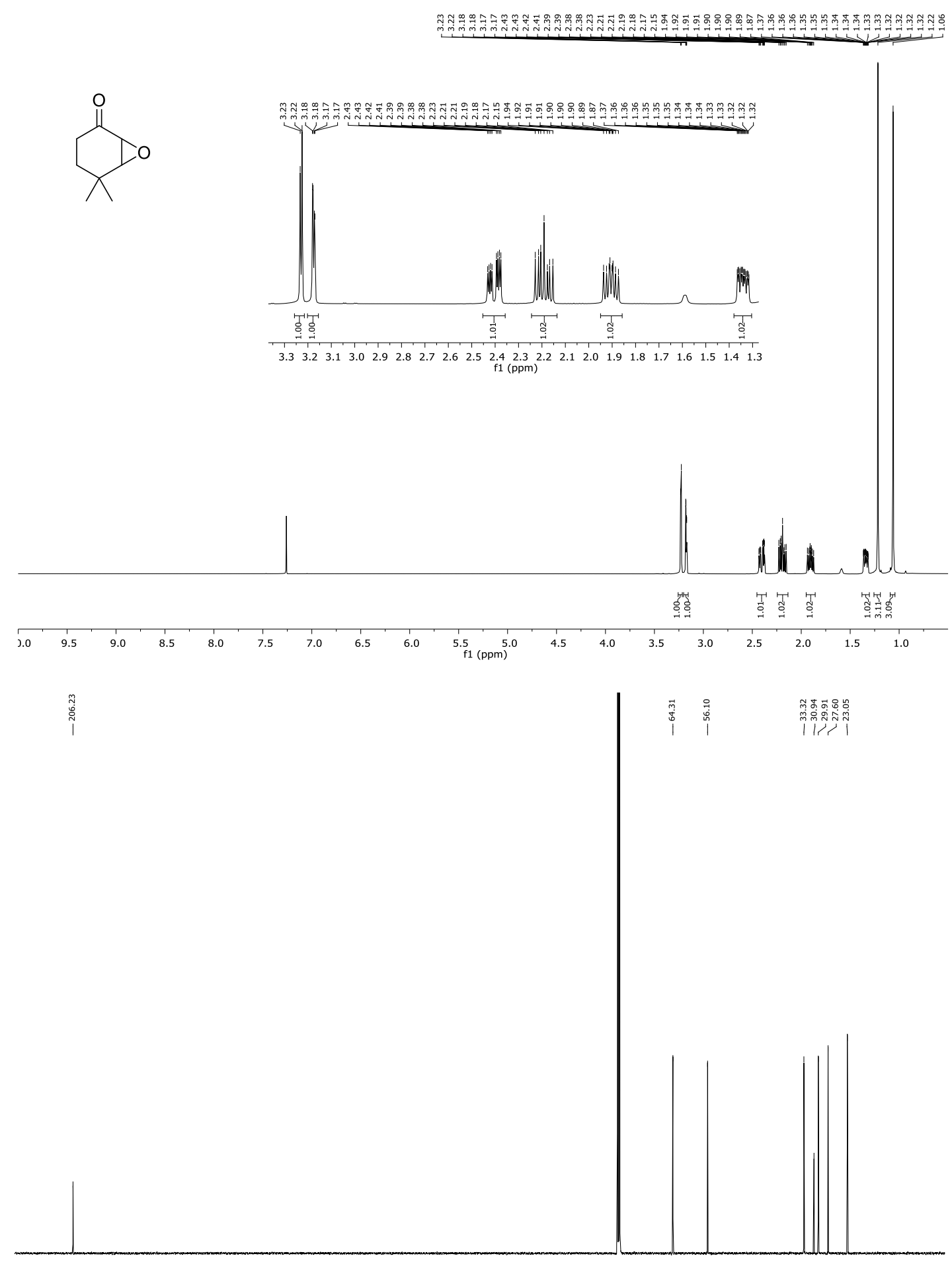

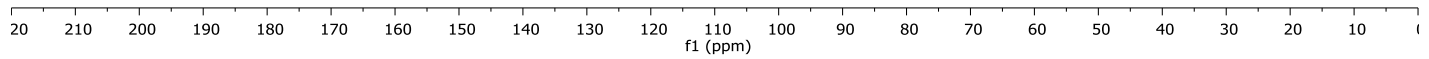


${ }^{1} \mathrm{H}(500 \mathrm{MHz})$ and ${ }^{13} \mathrm{C}\left\{{ }^{1} \mathrm{H}\right\}$ NMR (101 MHz) spectra of compound $\mathbf{8 a}\left(\mathrm{CDCl}_{3}\right)$

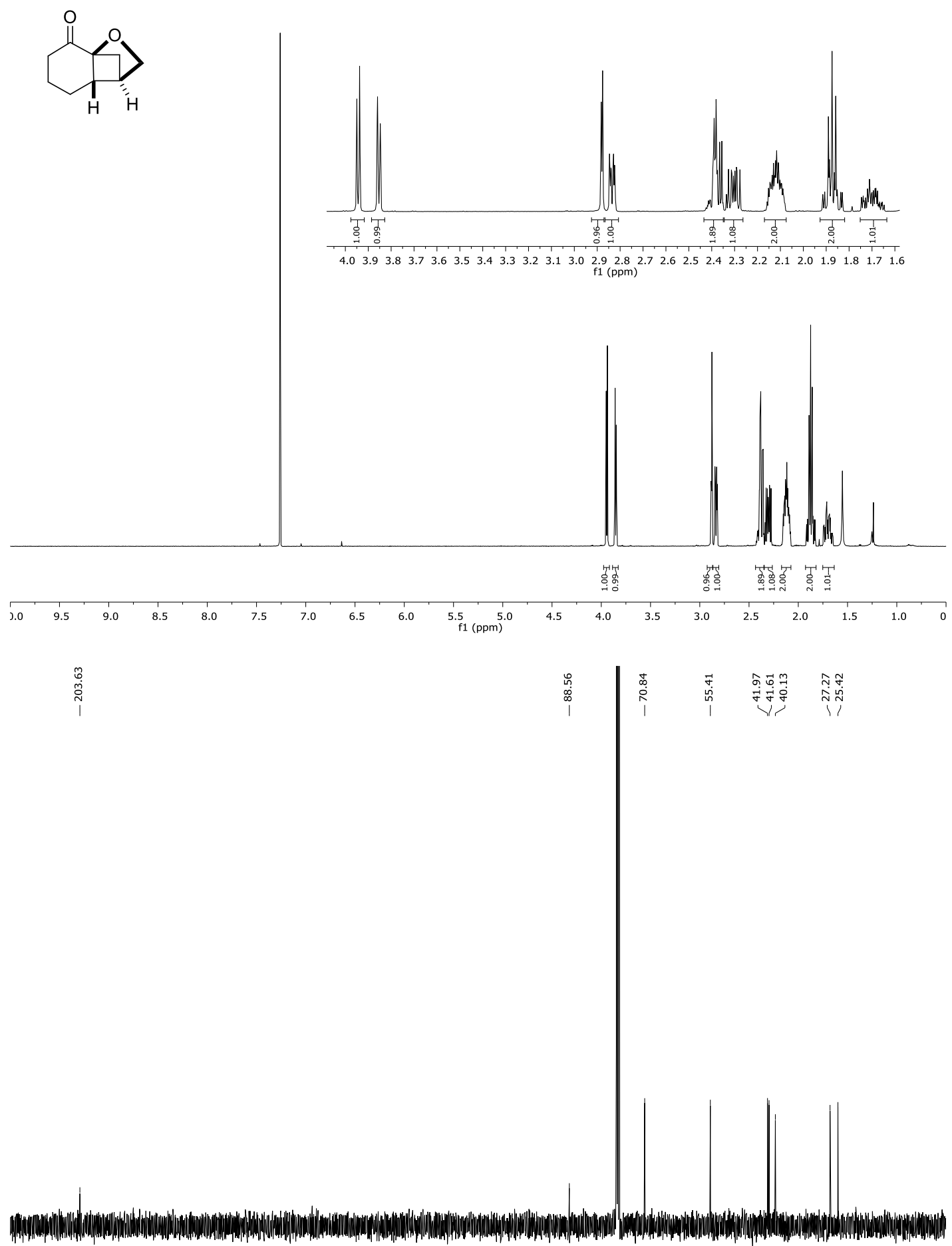

\begin{tabular}{lllllllllllllllllllllll}
\hline & 210 & 200 & 190 & 180 & 170 & 160 & 150 & 140 & 130 & 120 & 110 & 100 & 90 & 80 & 70 & 60 & 50 & 40 & 30 & 20 & 10 & 1
\end{tabular} 
${ }^{1} \mathrm{H}(400 \mathrm{MHz})$ and ${ }^{13} \mathrm{C}\left\{{ }^{1} \mathrm{H}\right\}$ NMR (126 MHz) spectra of compound $\mathbf{8 b}\left(\mathrm{CDCl}_{3}\right)$

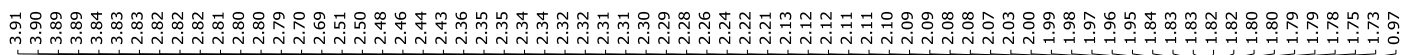<smiles>CC12CCCC(=O)OC1CC2</smiles>
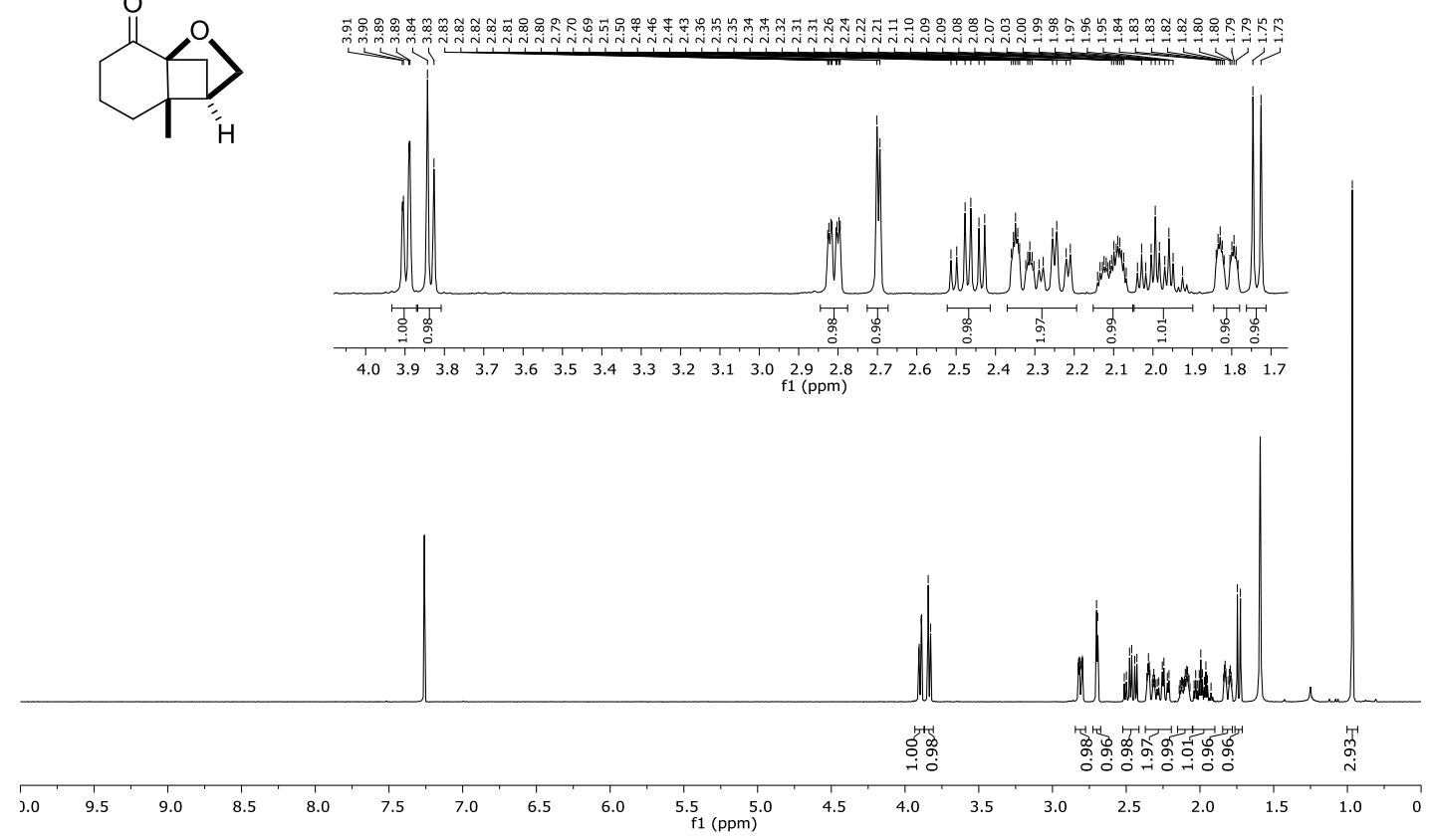
${ }^{1} \mathrm{H}(500 \mathrm{MHz})$ and ${ }^{13} \mathrm{C}\left\{{ }^{1} \mathrm{H}\right\}$ NMR (126 MHz) spectra of compound $8 \mathbf{c}\left(\mathrm{CDCl}_{3}\right)$

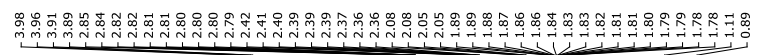<smiles>CC1(C)CC(=O)C23CC(CO2)[C@H]3C1</smiles>
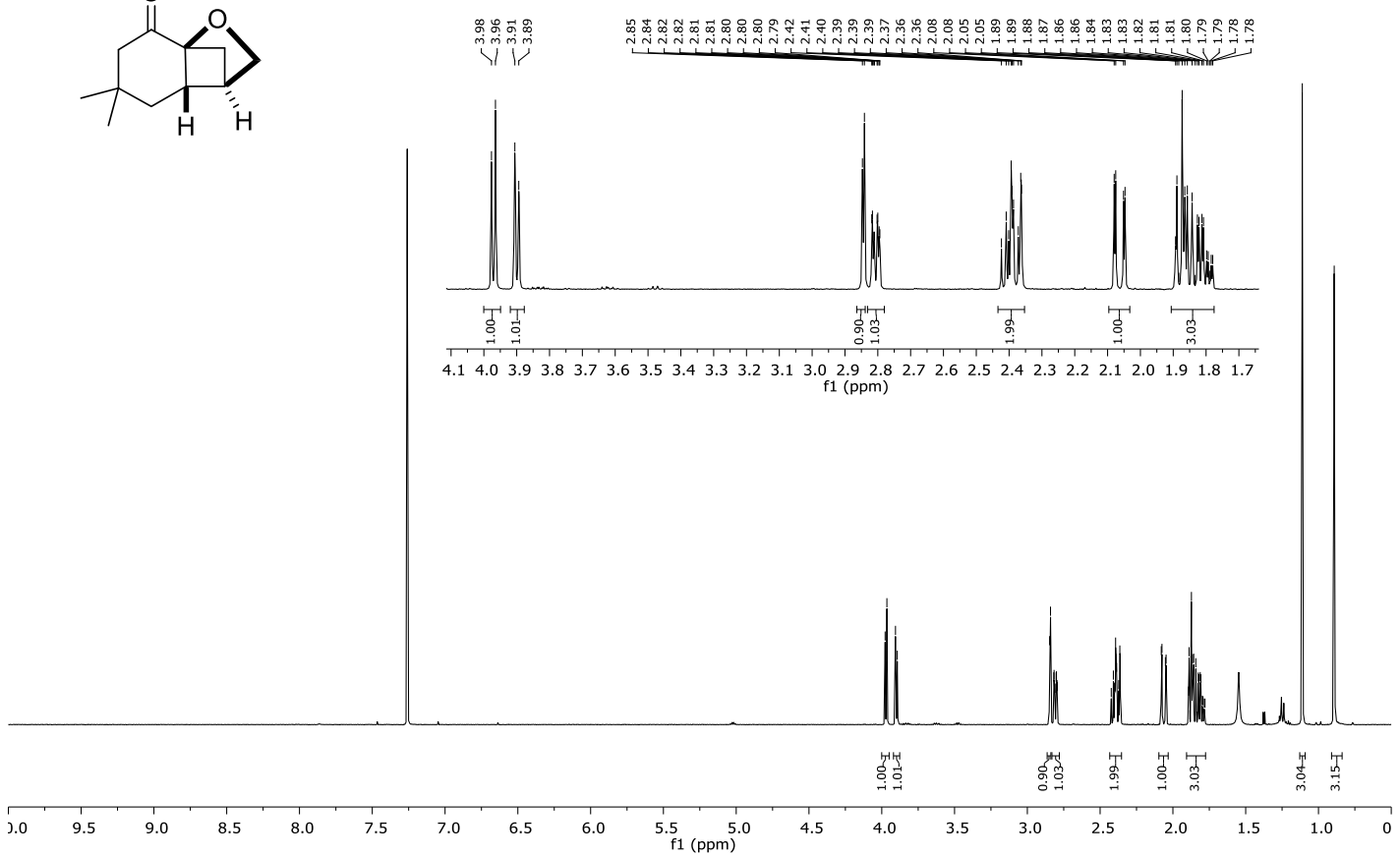

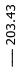

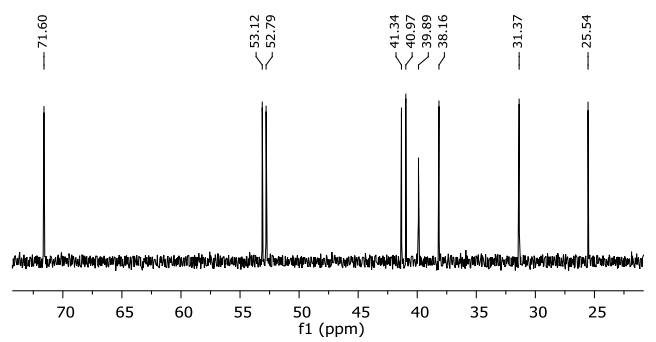

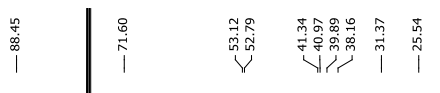

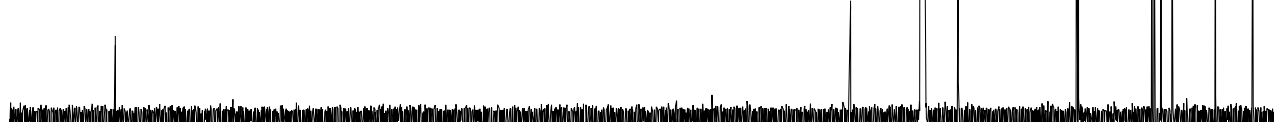

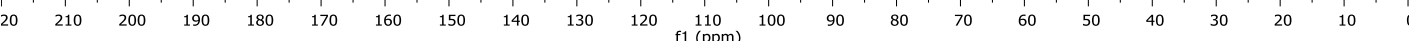


${ }^{1} \mathrm{H}(500 \mathrm{MHz})$ and ${ }^{13} \mathrm{C}\left\{{ }^{1} \mathrm{H}\right\}$ NMR (101 MHz) spectra of compound $\mathbf{9 a}\left(\mathrm{CDCl}_{3}\right)$

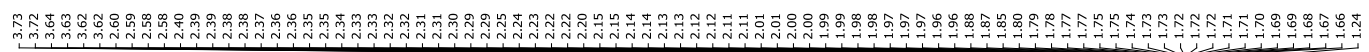
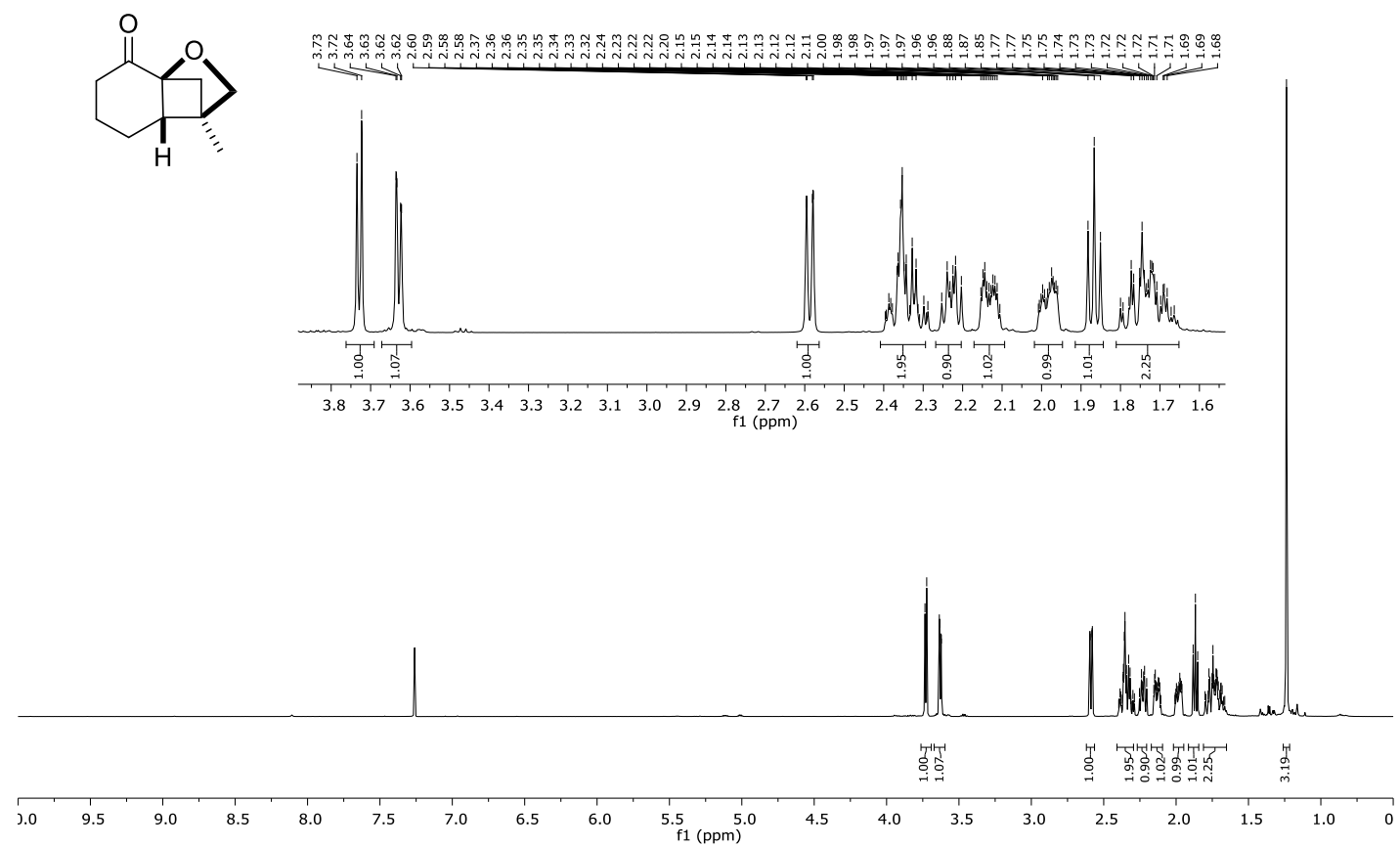
${ }^{1} \mathrm{H}(500 \mathrm{MHz})$ and ${ }^{13} \mathrm{C}\left\{{ }^{1} \mathrm{H}\right\} \mathrm{NMR}(126 \mathrm{MHz})$ spectra of compound $\mathbf{9 b}\left(\mathrm{CDCl}_{3}\right)$

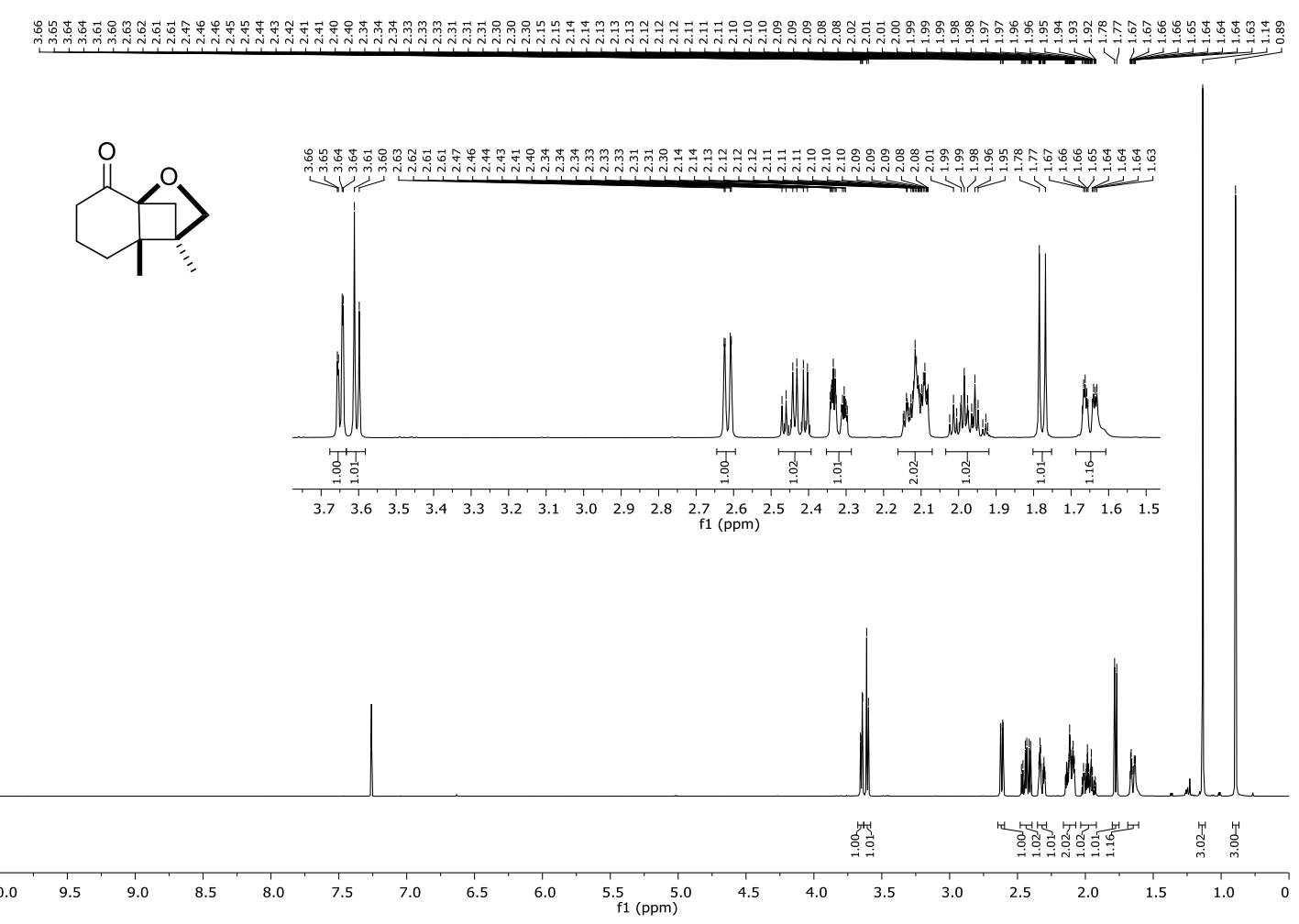

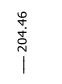
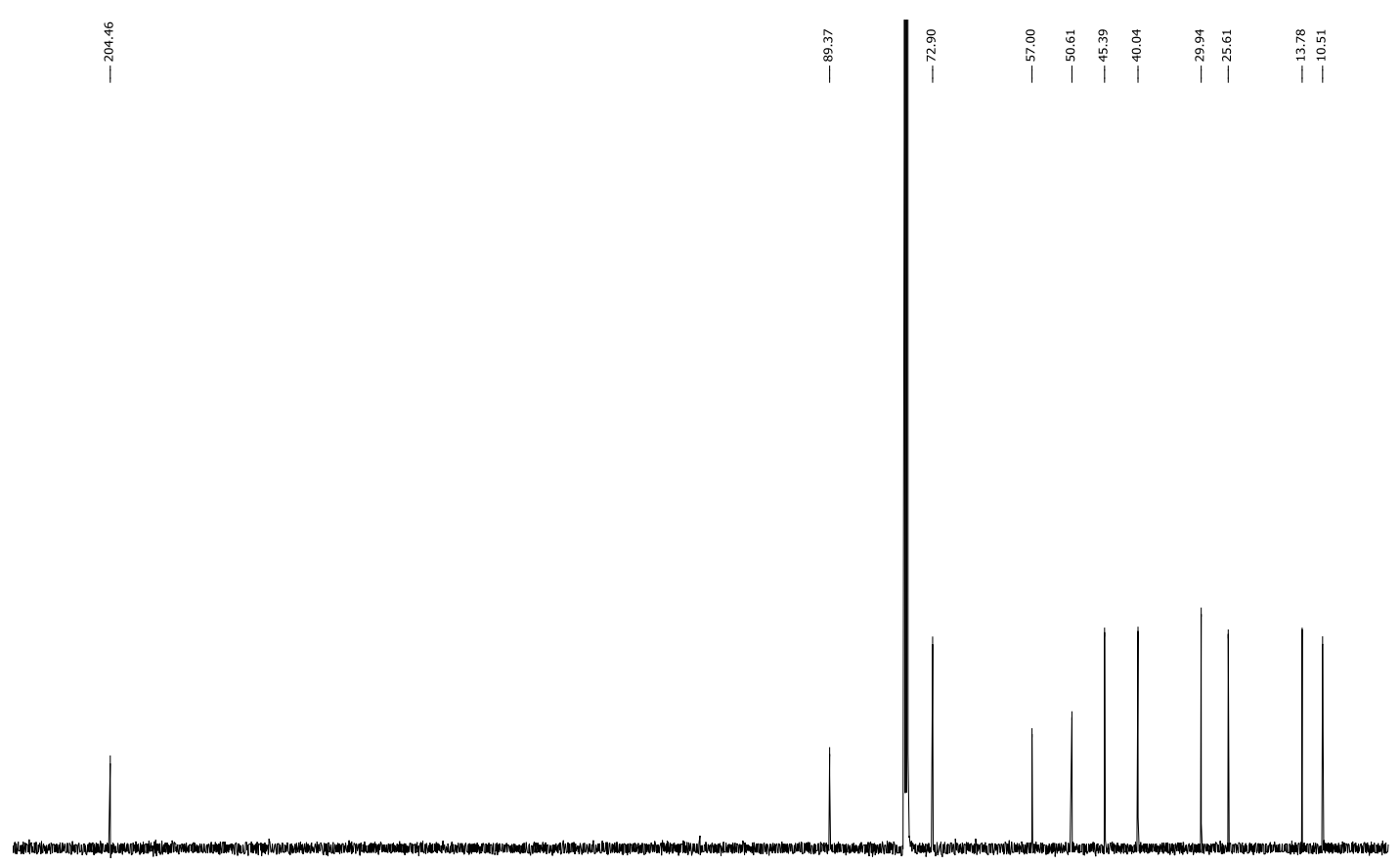

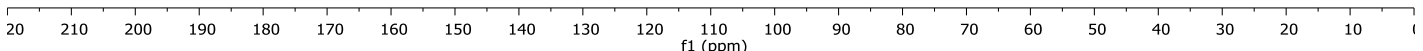


${ }^{1} \mathrm{H}(500 \mathrm{MHz})$ and ${ }^{13} \mathrm{C}\left\{{ }^{1} \mathrm{H}\right\}$ NMR (126 MHz) spectra of compound $9 \mathbf{c}\left(\mathrm{CDCl}_{3}\right)$
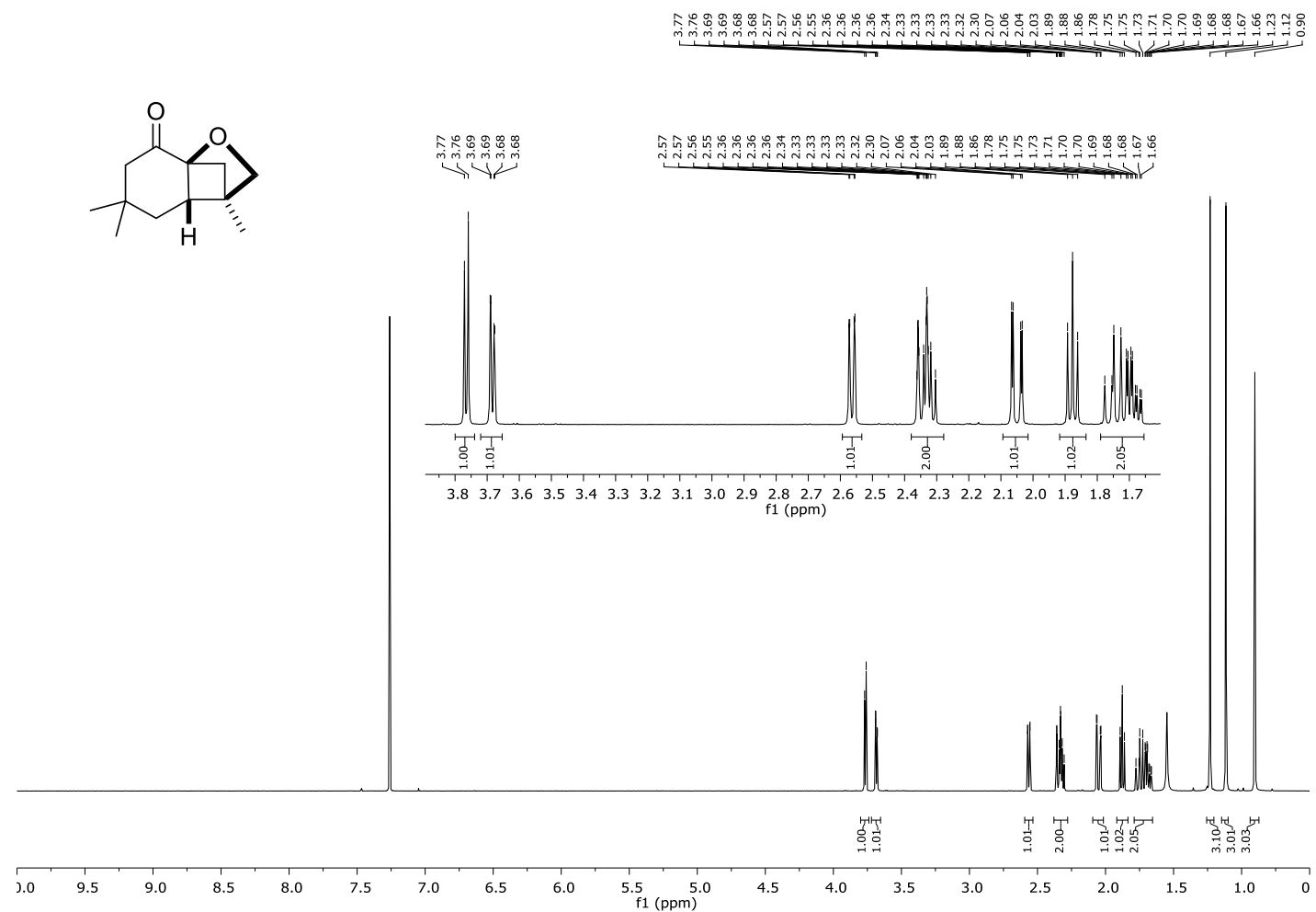

ֻั
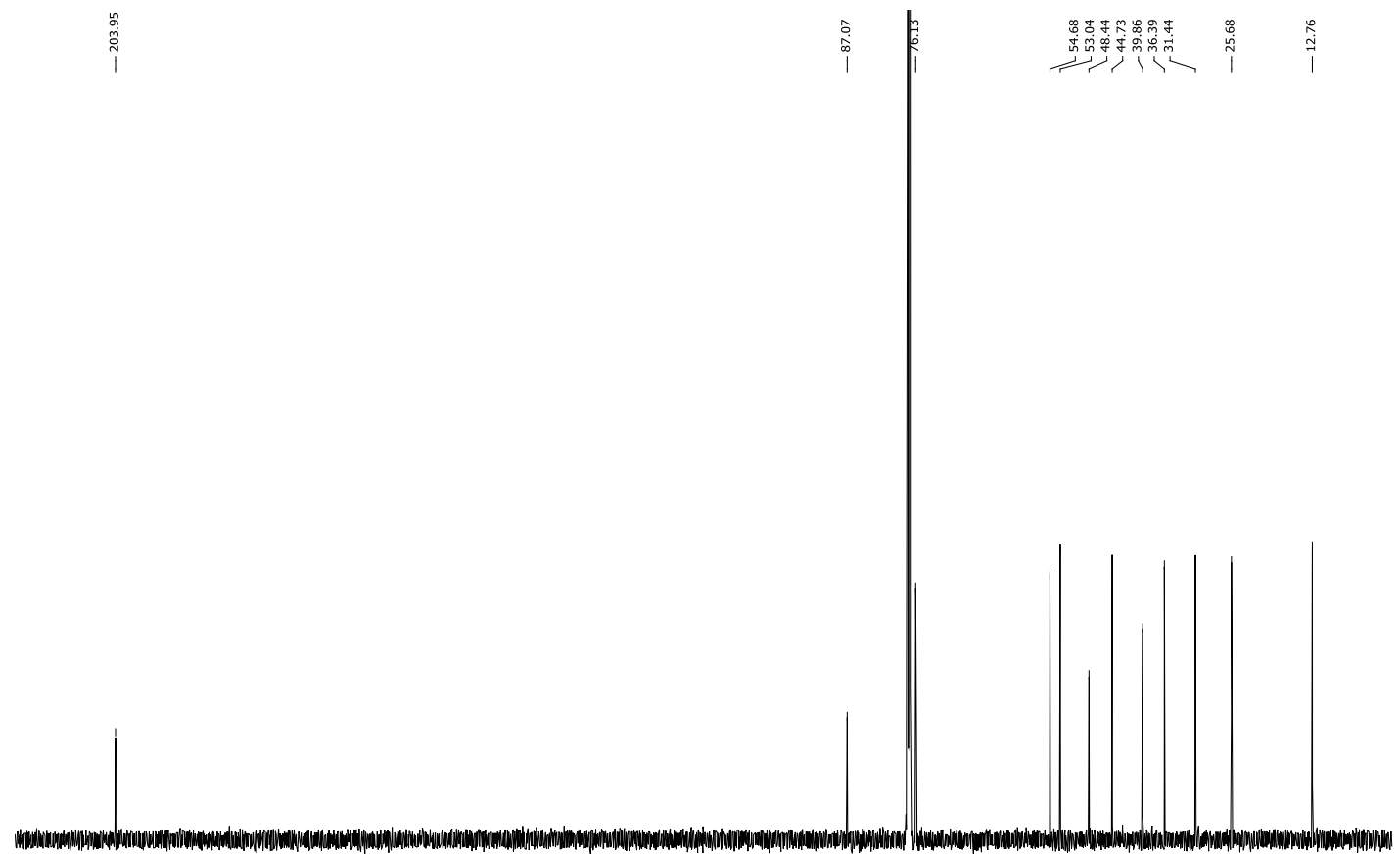

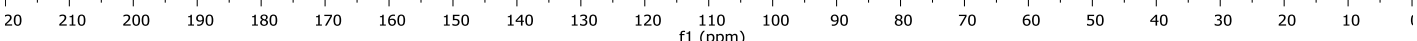


${ }^{1} \mathrm{H}(500 \mathrm{MHz})$ and ${ }^{13} \mathrm{C}\left\{{ }^{1} \mathrm{H}\right\} \mathrm{NMR}(126 \mathrm{MHz})$ spectra of compound $\mathbf{9 d}\left(\mathrm{CDCl}_{3}\right)$

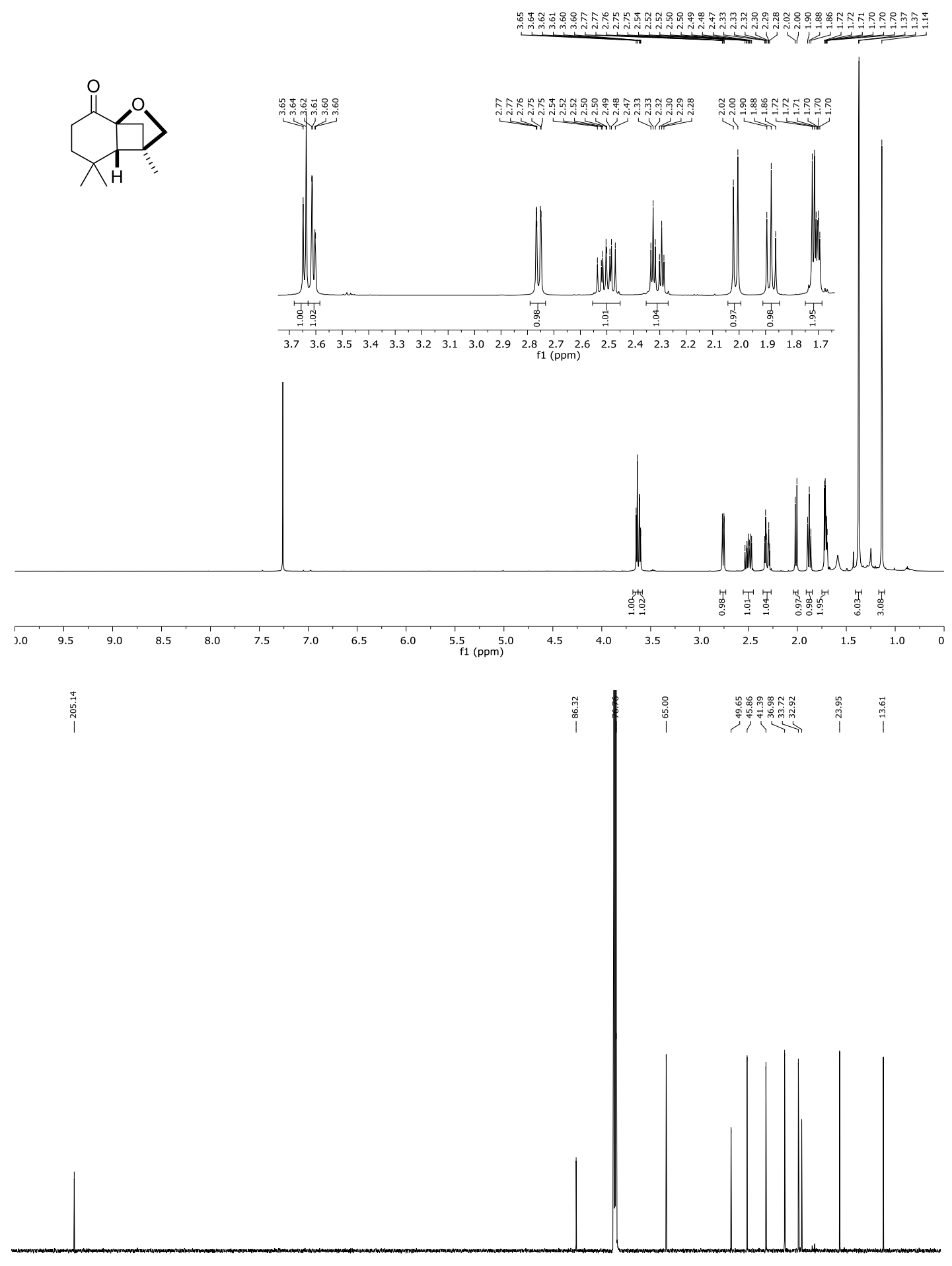

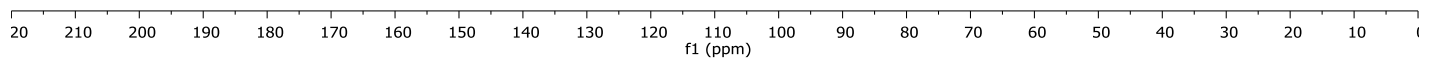


${ }^{1} \mathrm{H}(500 \mathrm{MHz})$ and ${ }^{13} \mathrm{C}\left\{{ }^{1} \mathrm{H}\right\}$ NMR $(126 \mathrm{MHz})$ spectra of compound $9 \mathbf{e}\left(\mathrm{CDCl}_{3}\right)$

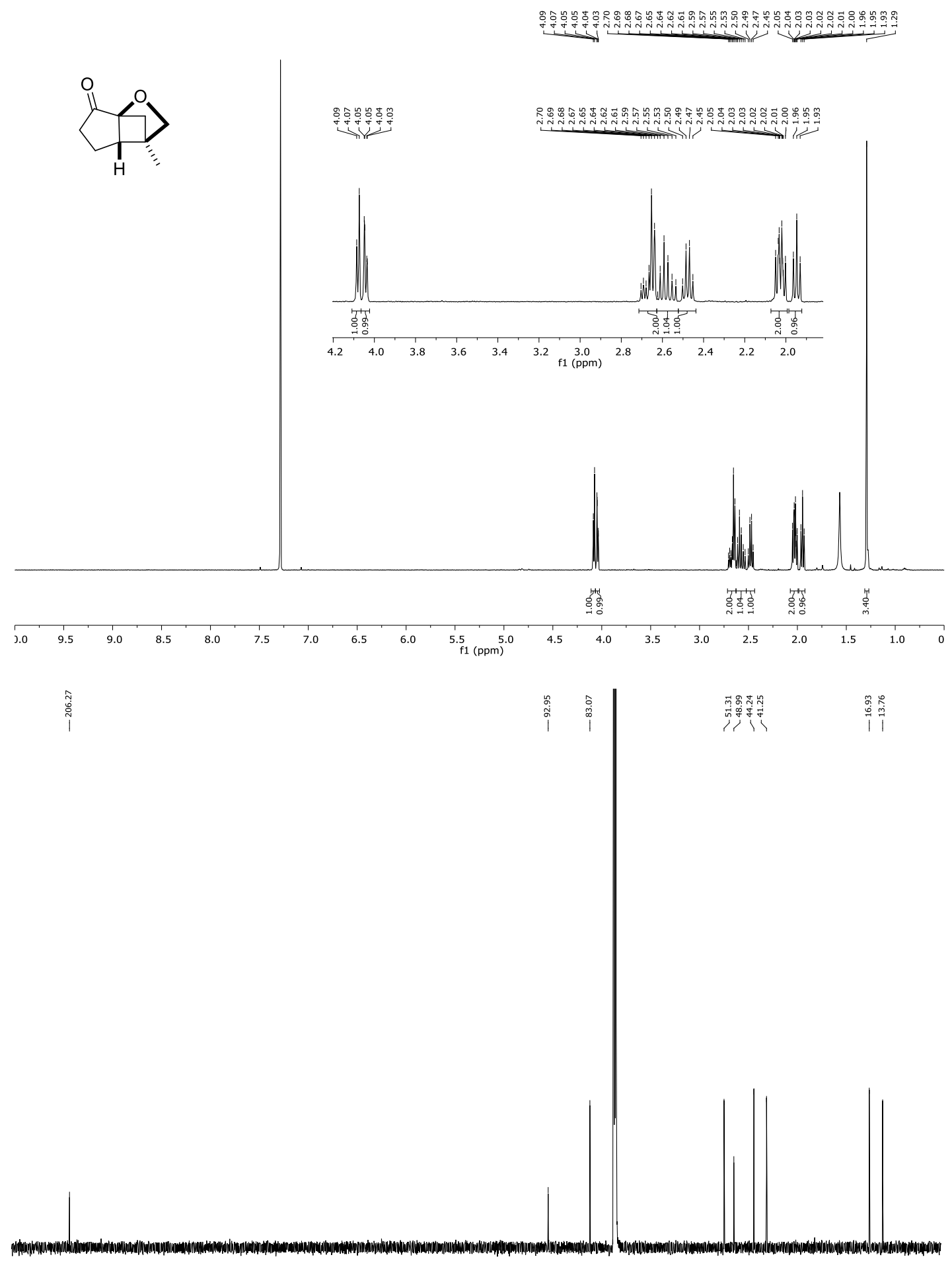

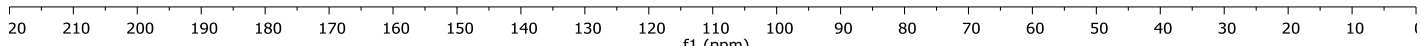


${ }^{1} \mathrm{H}(400 \mathrm{MHz})$ and ${ }^{13} \mathrm{C}\left\{{ }^{1} \mathrm{H}\right\}$ NMR (101 MHz) spectra of compound $E-\mathbf{1 0} / \mathrm{Z}-\mathbf{1 0}\left(\mathrm{CDCl}_{3}\right)$

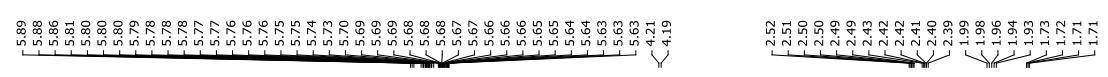
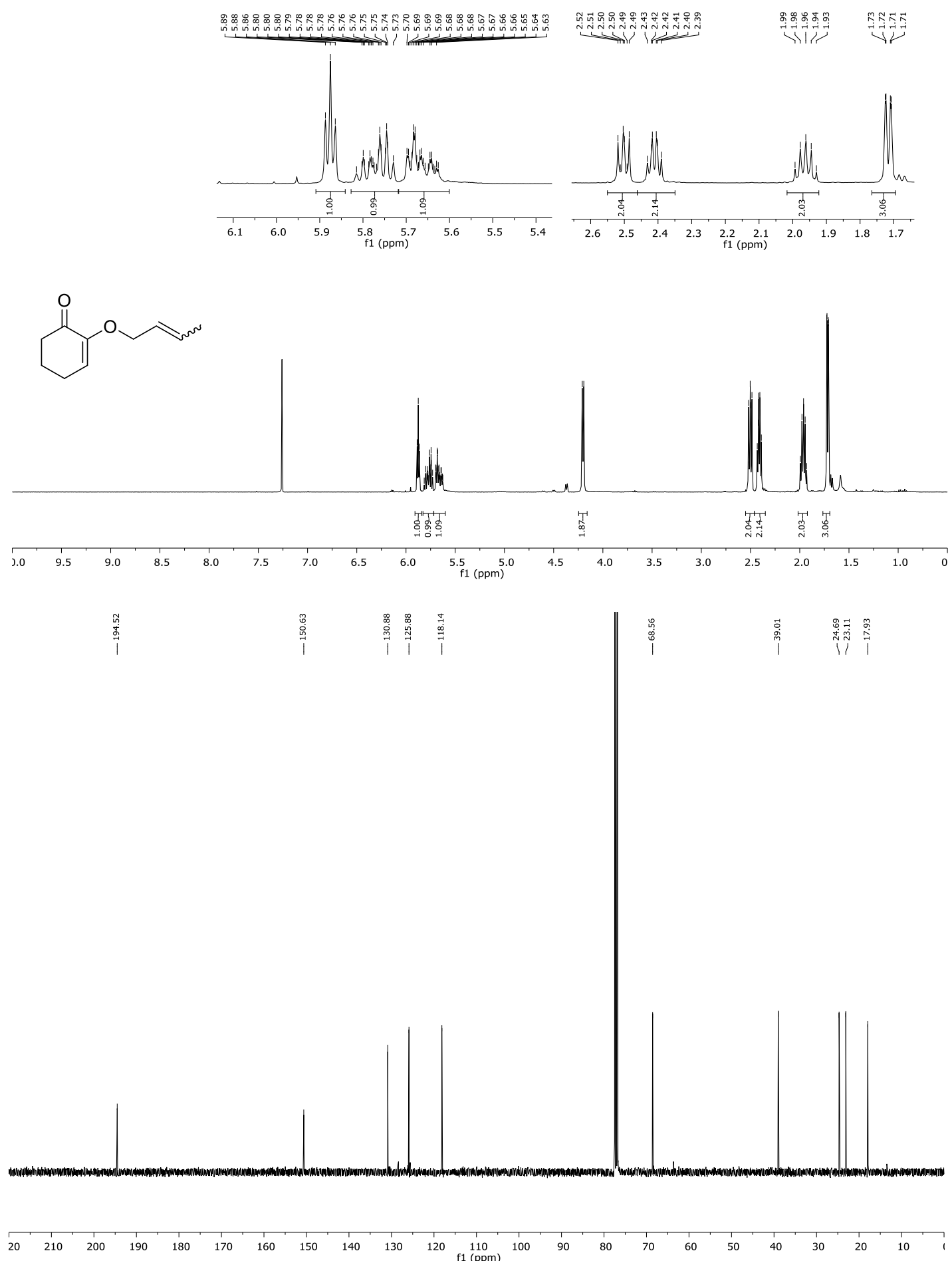
${ }^{1} \mathrm{H}(500 \mathrm{MHz})$ and ${ }^{13} \mathrm{C}\left\{{ }^{1} \mathrm{H}\right\}$ NMR (126 MHz) spectra of compound $11\left(\mathrm{CDCl}_{3}\right)$

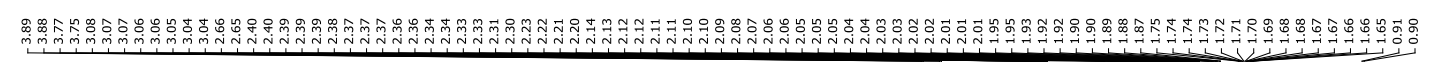

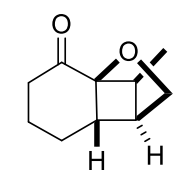
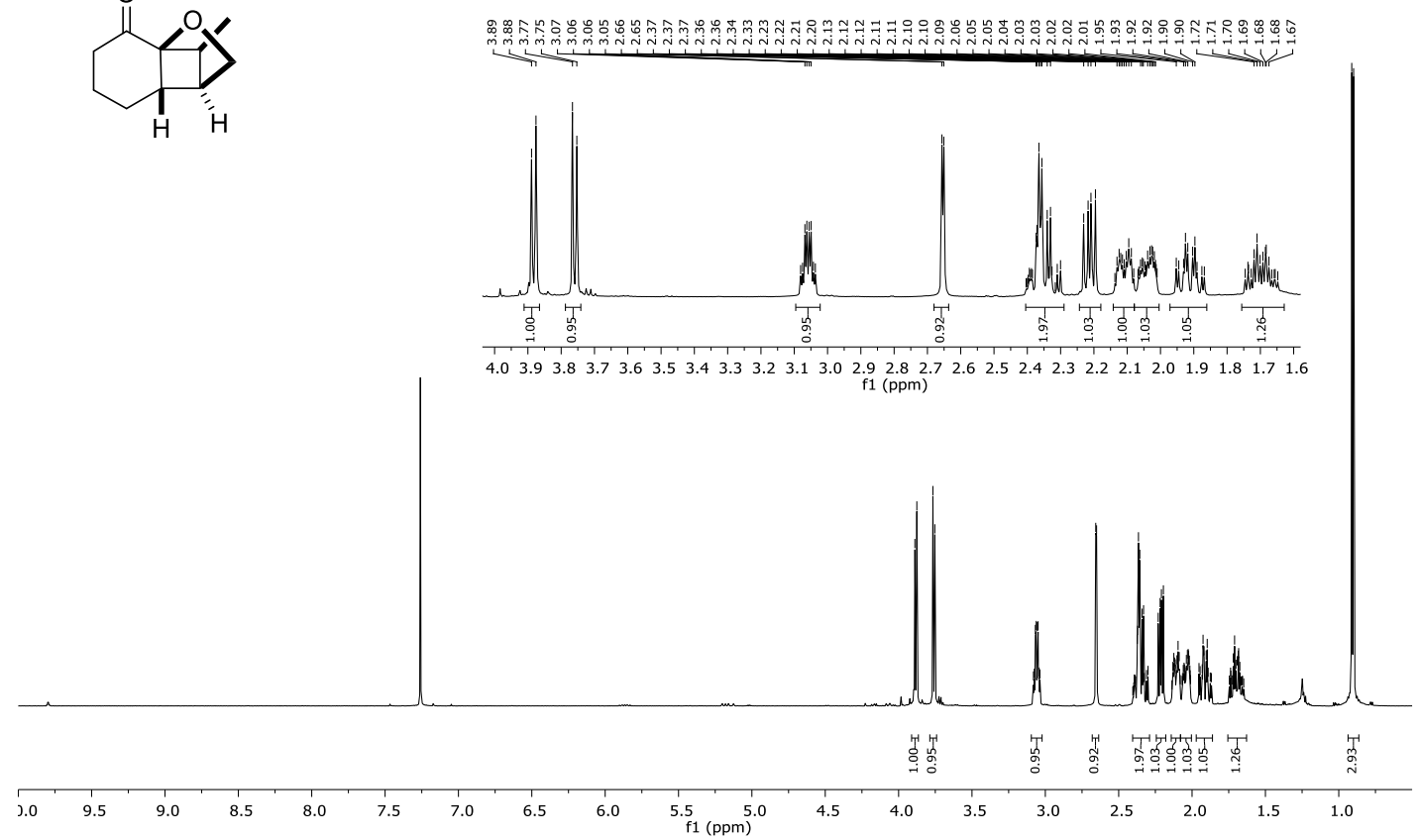

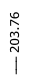
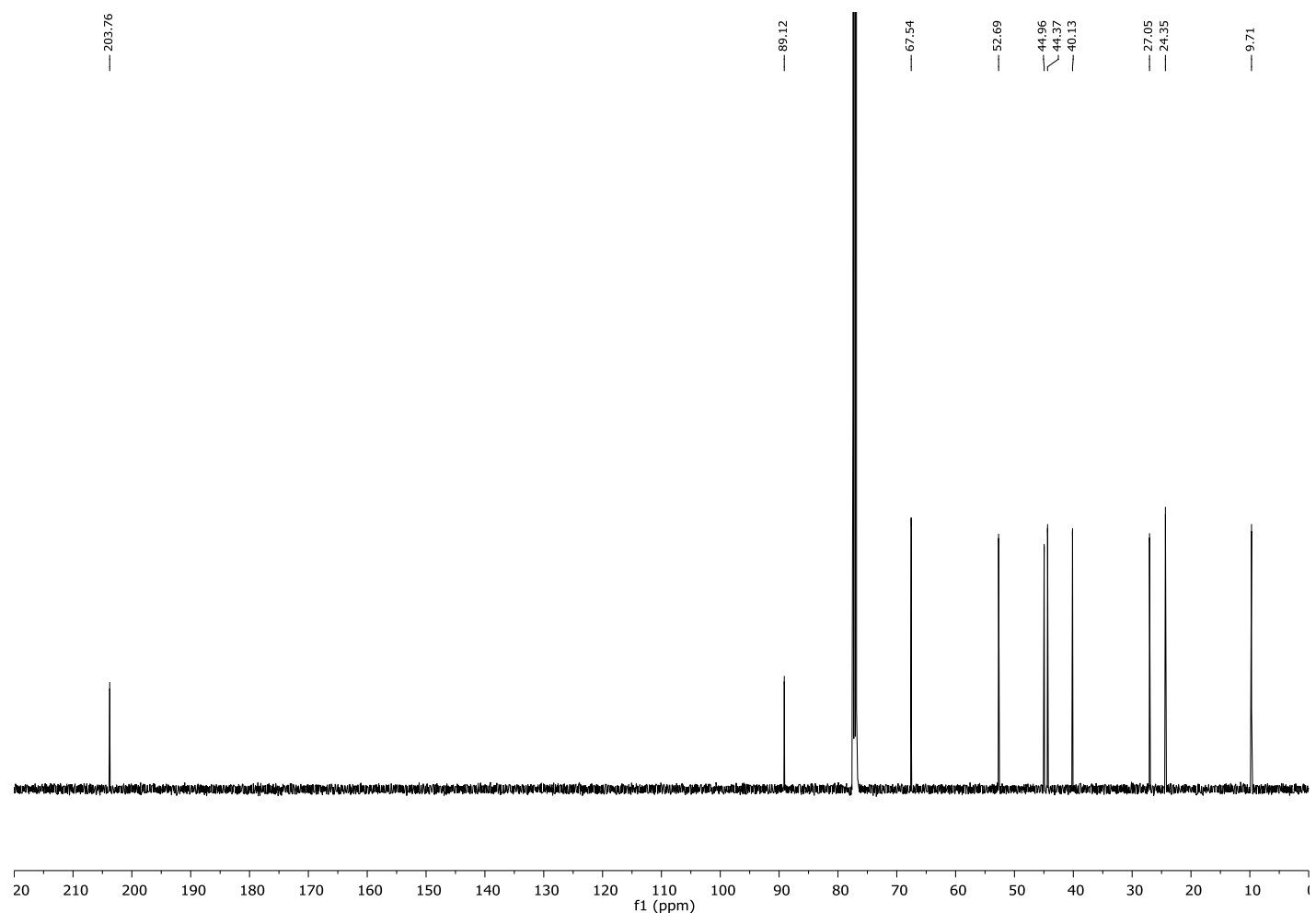
${ }^{1} \mathrm{H}(500 \mathrm{MHz})$ and ${ }^{13} \mathrm{C}\left\{{ }^{1} \mathrm{H}\right\} \mathrm{NMR}(126 \mathrm{MHz})$ spectra of compound $E-\mathbf{1 2}\left(\mathrm{CDCl}_{3}\right)$

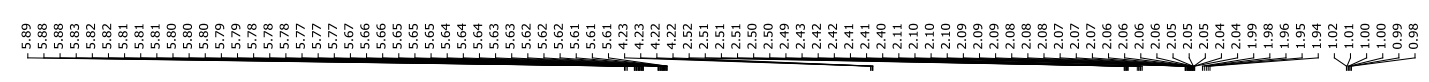
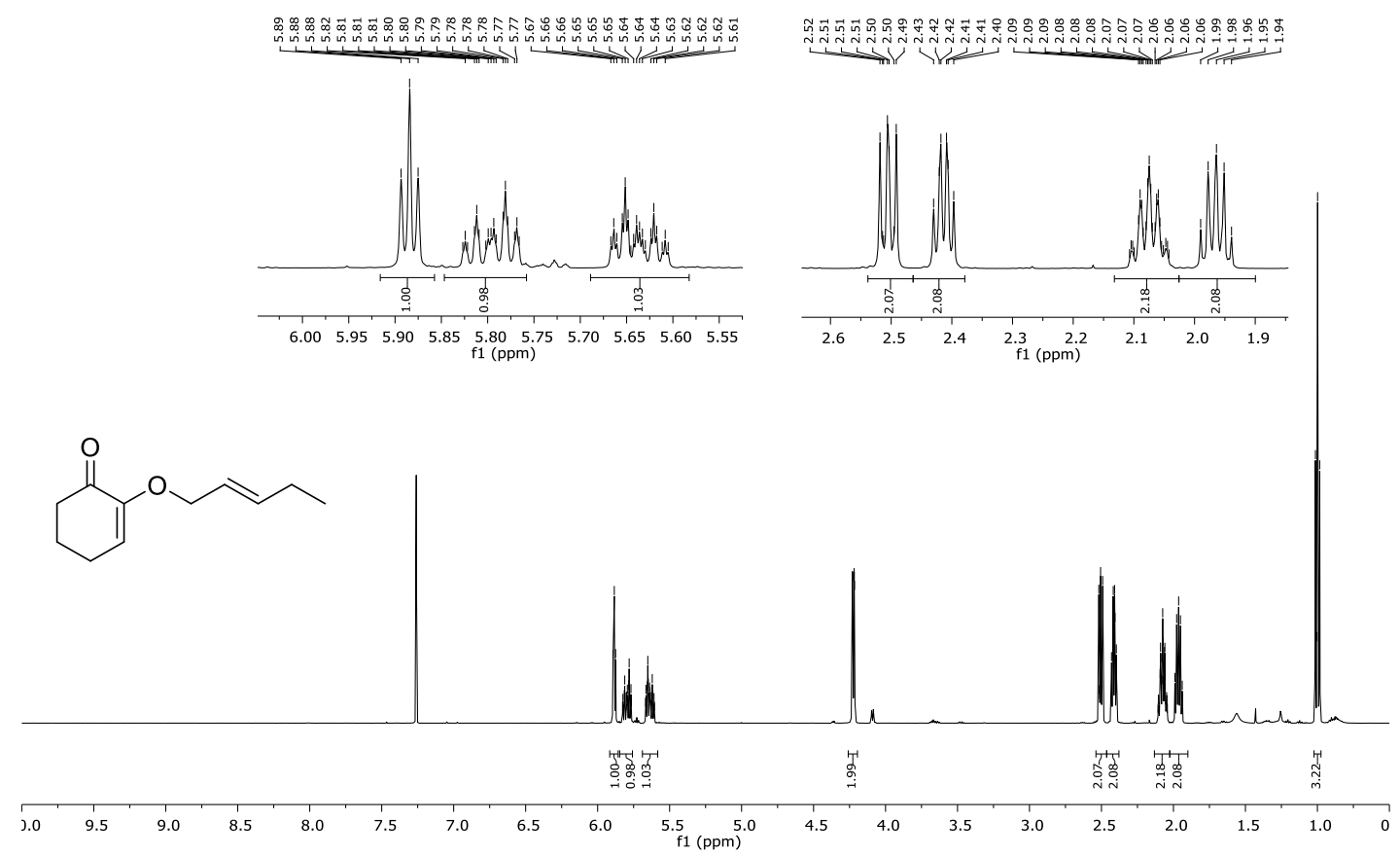

总
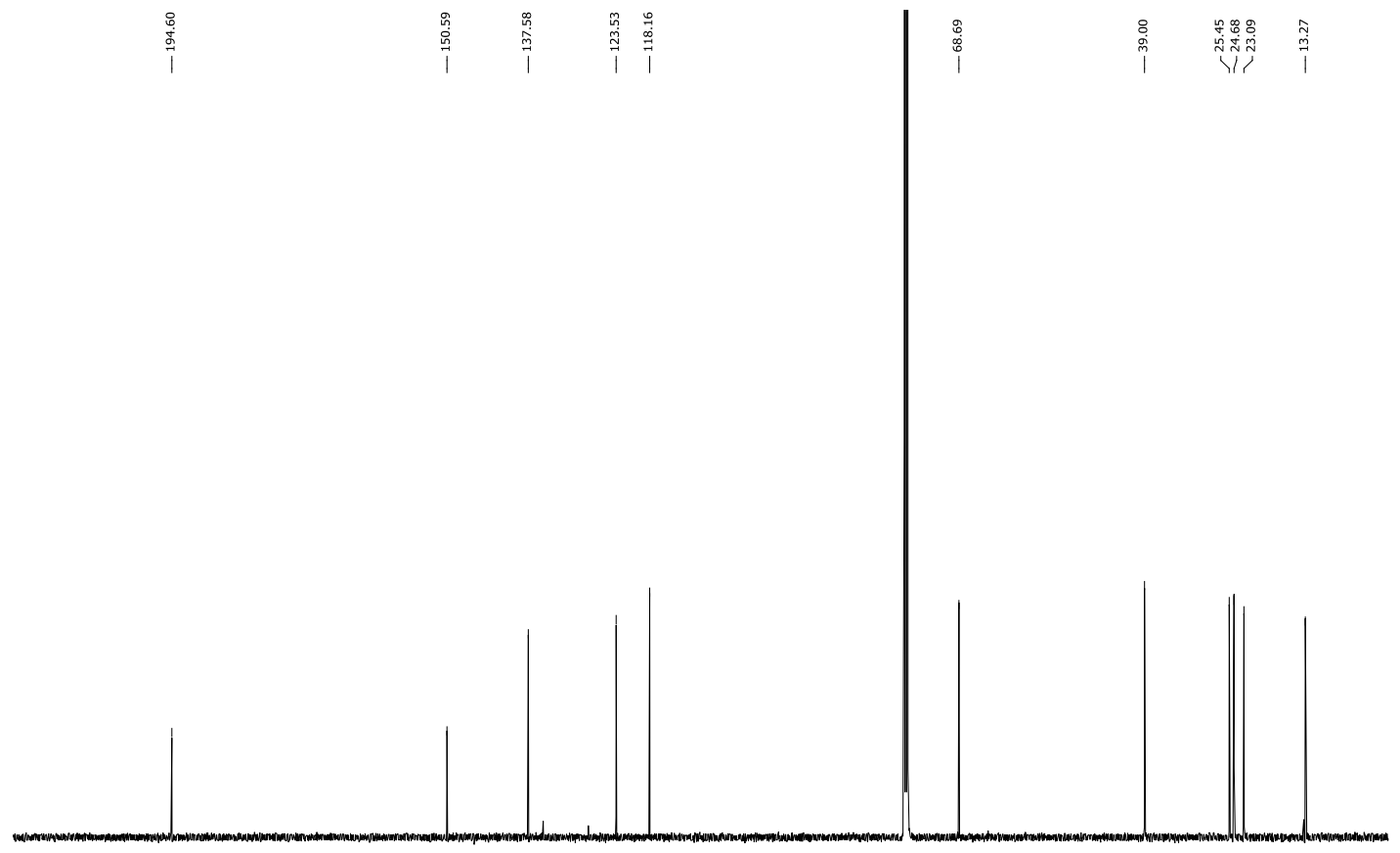

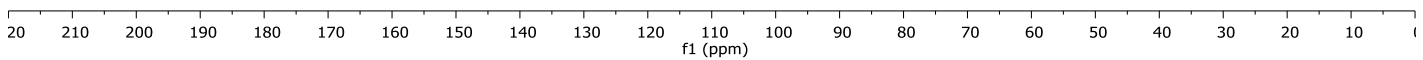


${ }^{1} \mathrm{H}(500 \mathrm{MHz})$ and ${ }^{13} \mathrm{C}\left\{{ }^{1} \mathrm{H}\right\} \mathrm{NMR}(75 \mathrm{MHz})$ spectra of $(\boldsymbol{Z})$-1-Bromopent-2-ene $\left(\mathrm{CDCl}_{3}\right)$ $\underbrace{\text { Ring }}$ $\mathcal{B r}$
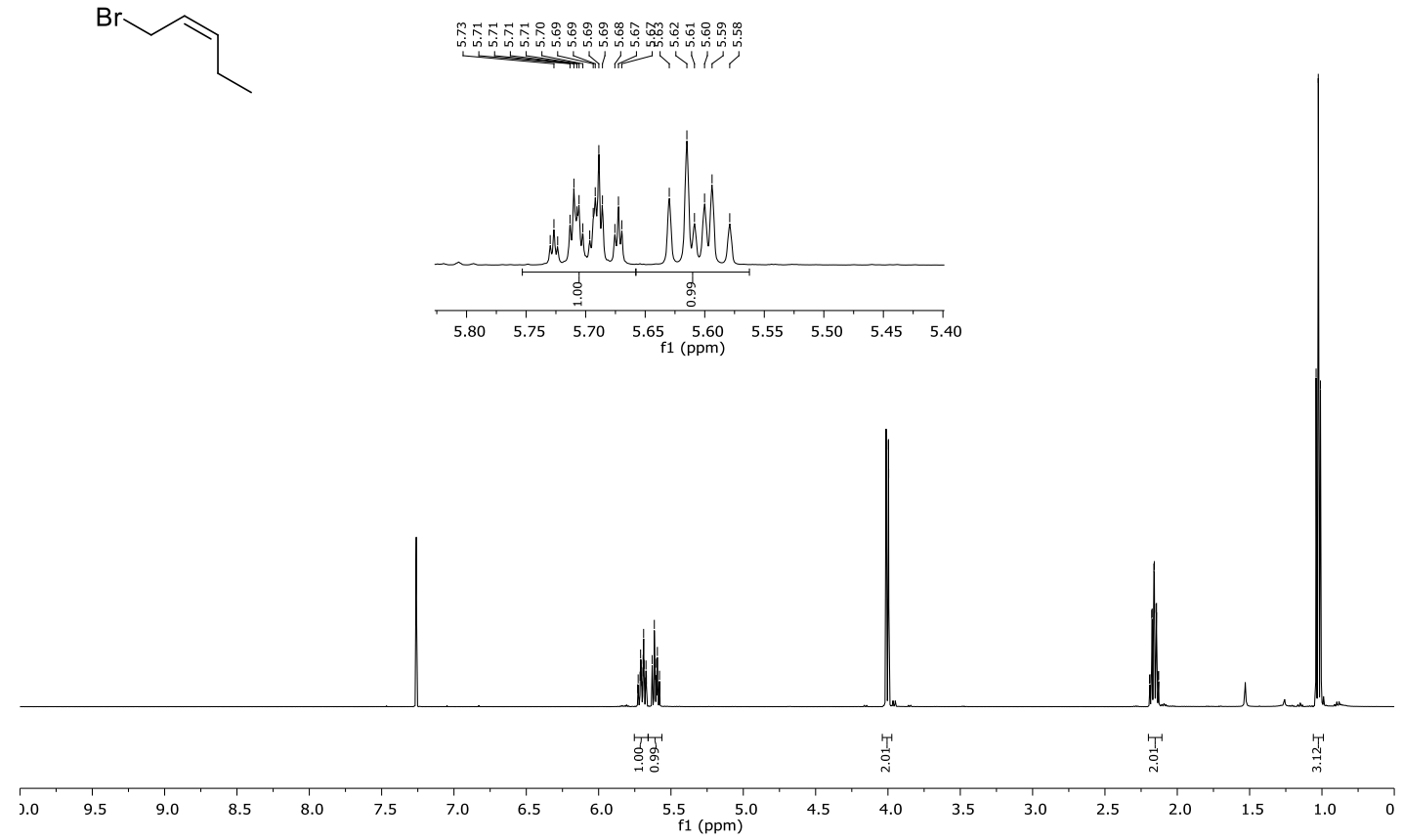
${ }^{1} \mathrm{H}(500 \mathrm{MHz})$ and ${ }^{13} \mathrm{C}\left\{{ }^{1} \mathrm{H}\right\} \mathrm{NMR}(126 \mathrm{MHz})$ spectra of compound $\mathrm{Z}-\mathbf{1 2}\left(\mathrm{CDCl}_{3}\right)$

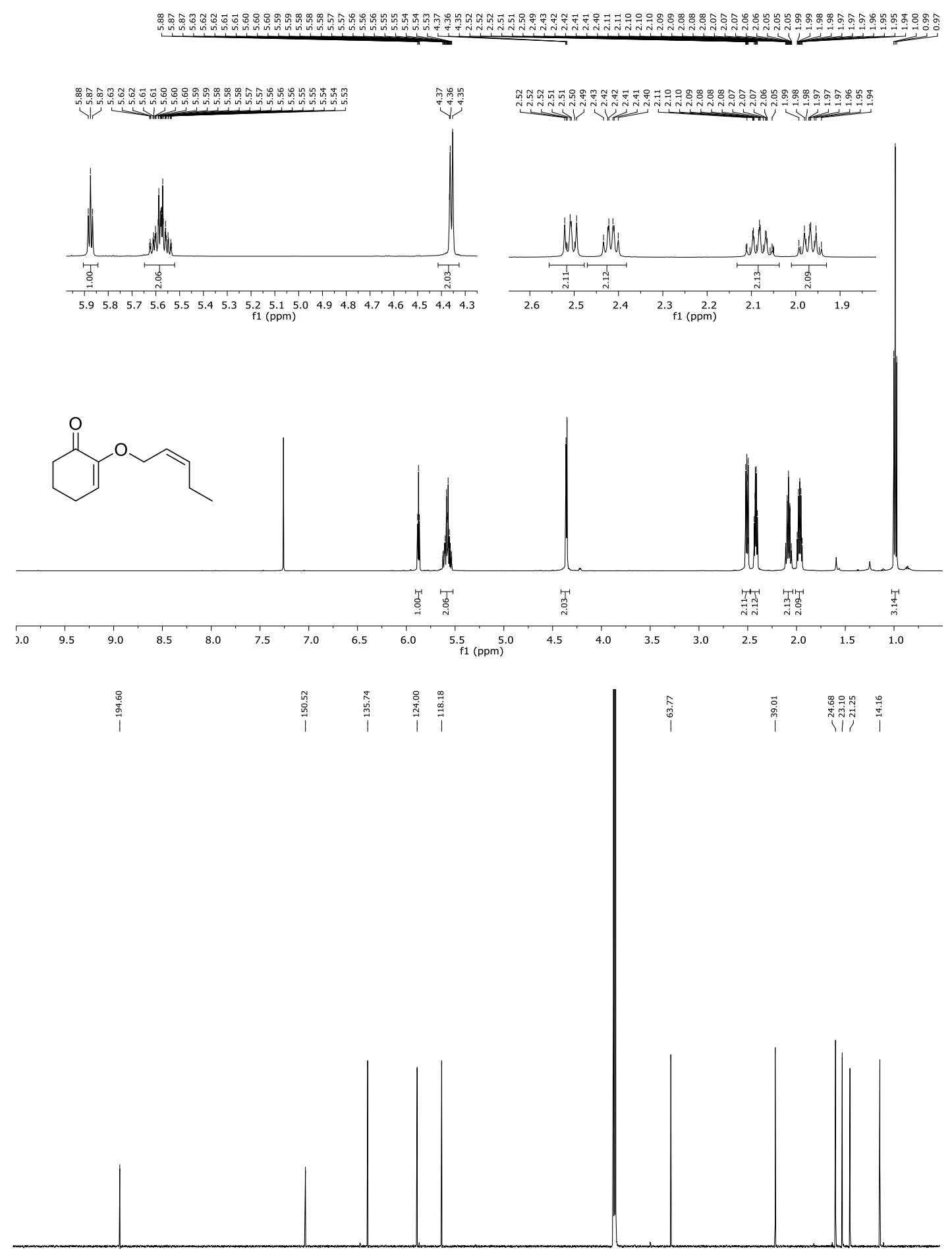

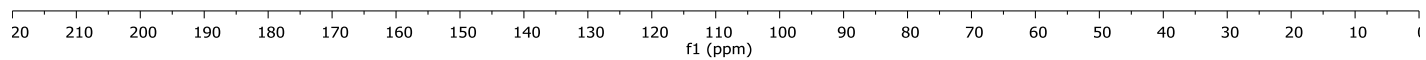


${ }^{1} \mathrm{H}(500 \mathrm{MHz})$ and ${ }^{13} \mathrm{C}\left\{{ }^{1} \mathrm{H}\right\}$ NMR $(126 \mathrm{MHz})$ spectra of compound $\mathbf{1 3}\left(\mathrm{CDCl}_{3}\right)$

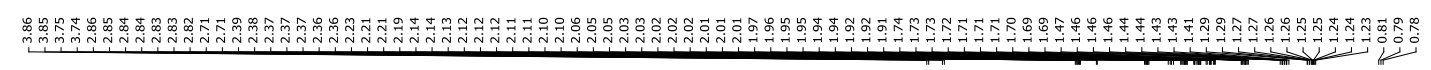

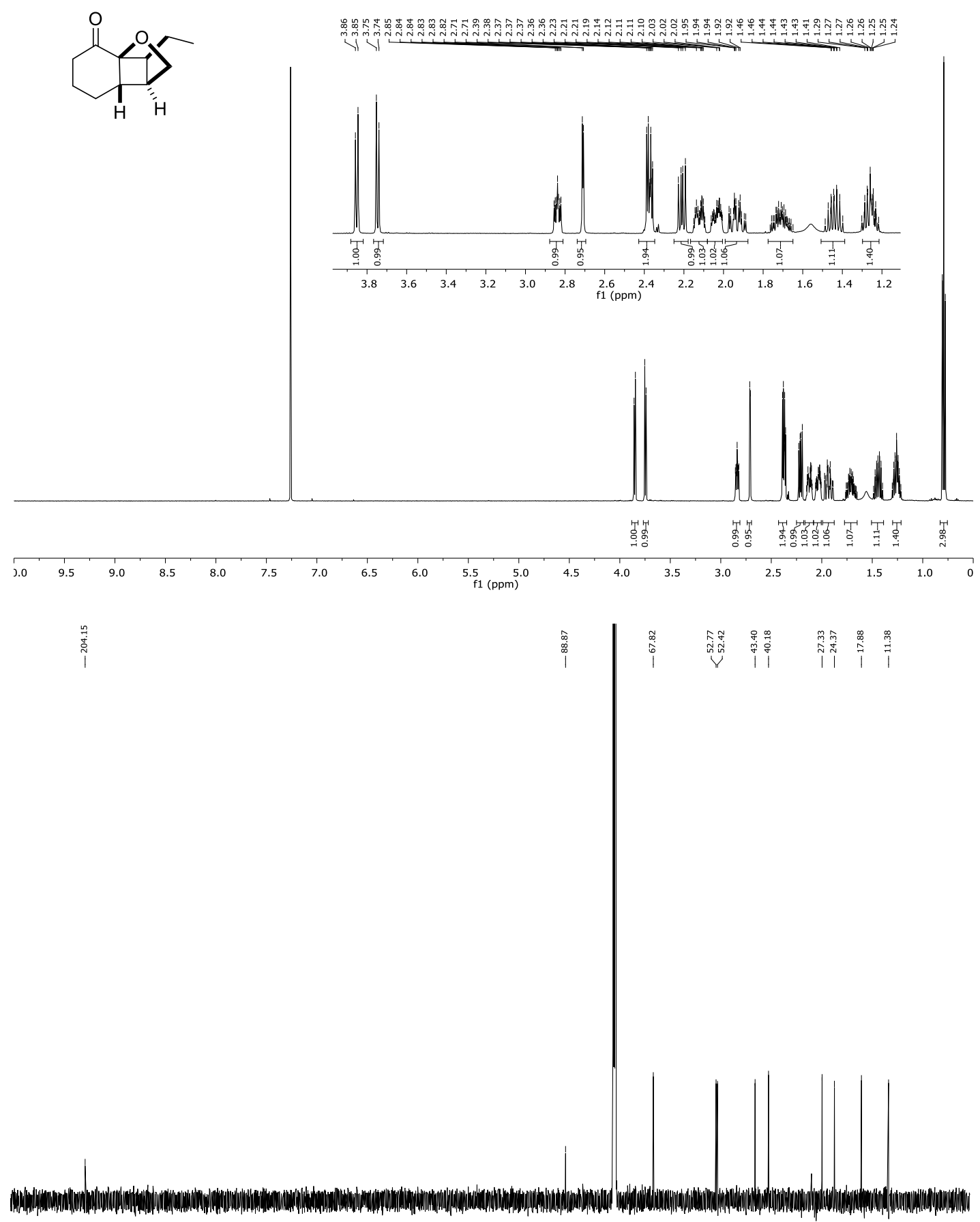

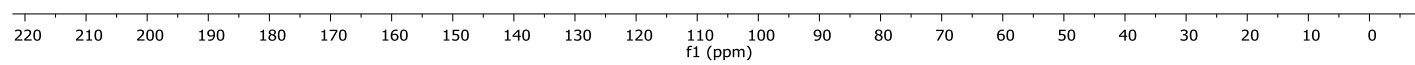


${ }^{1} \mathrm{H}(500 \mathrm{MHz})$ and ${ }^{13} \mathrm{C}\left\{{ }^{1} \mathrm{H}\right\}$ NMR $(75 \mathrm{MHz})$ spectra of compound 16a $\left(\mathrm{CDCl}_{3}\right)$

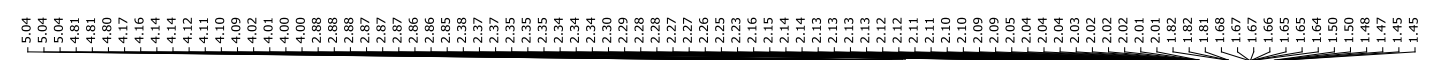<smiles>C=C(C)[C@@H]1CO[C@H]2C(=O)CCC[C@@H]12</smiles>
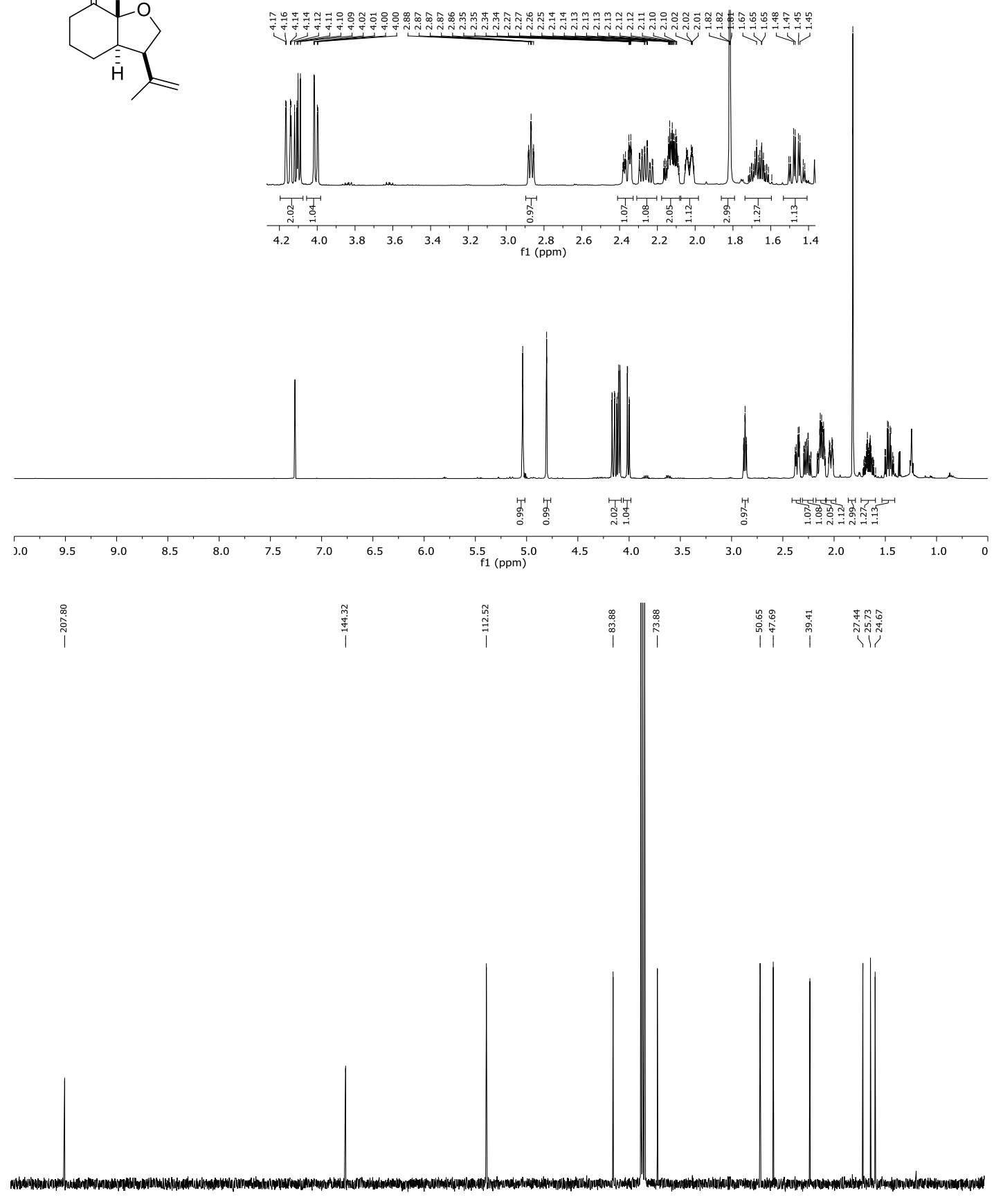

20
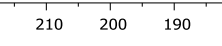

180

$150 \quad 140 \quad 130$

$20 \underset{\mathrm{f} 1(\mathrm{ppm})}{110} 100$

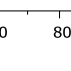

70 
${ }^{1} \mathrm{H}(400 \mathrm{MHz})$ and ${ }^{13} \mathrm{C}\left\{{ }^{1} \mathrm{H}\right\}$ NMR (126 MHz) spectra of compound $\mathbf{1 6 b}\left(\mathrm{CDCl}_{3}\right)$

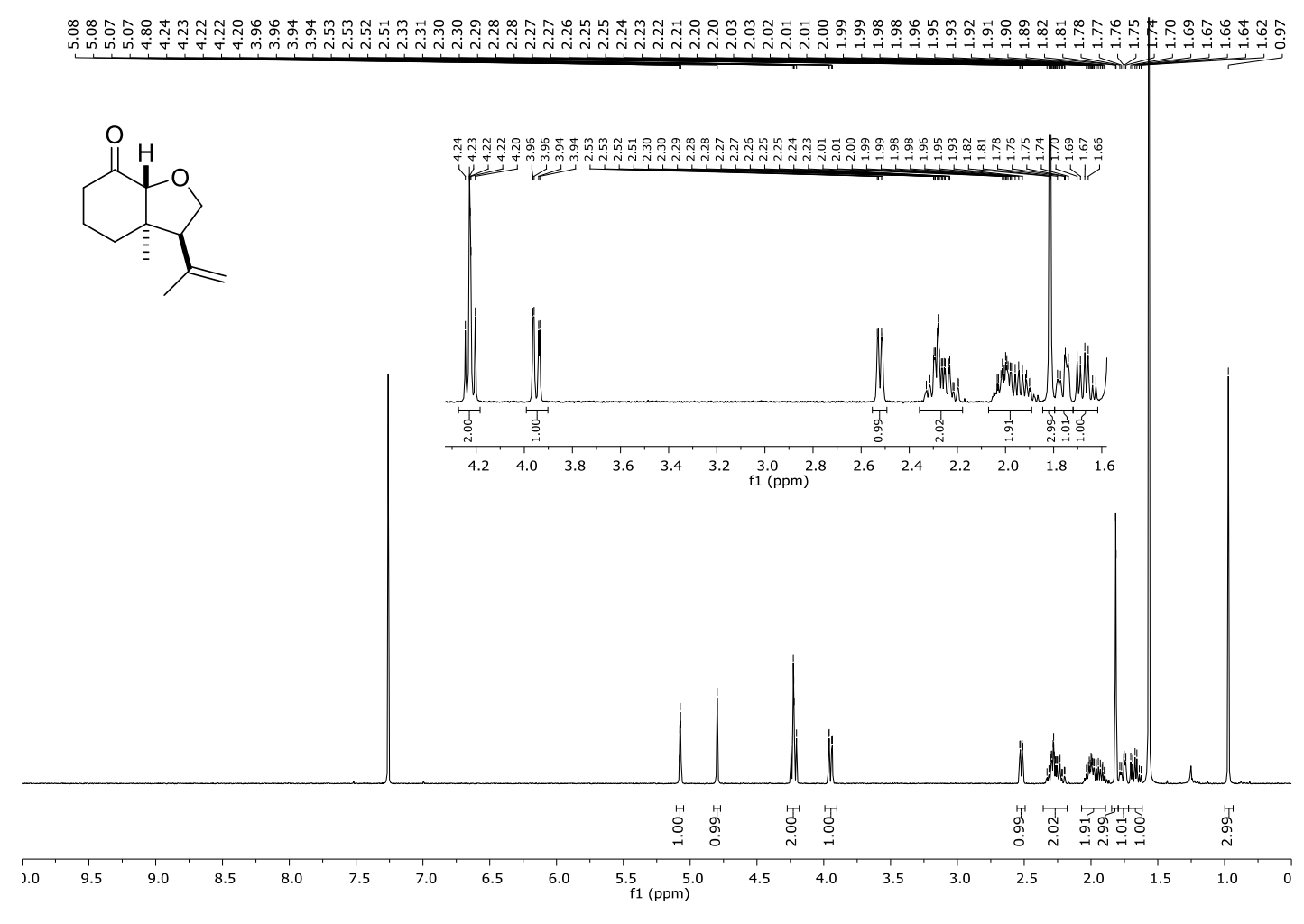

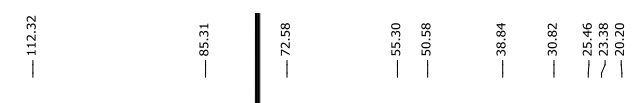

\begin{tabular}{llllllllllllllllllllll}
\hline 20 & 210 & 200 & 190 & 180 & 170 & 160 & 150 & 140 & 130 & 120 & 110 & 100 & 90 & 80 & 70 & 60 & 50 & 40 & 30 & 20 & 10 \\
\hline
\end{tabular} 
${ }^{1} \mathrm{H}(500 \mathrm{MHz})$ and ${ }^{13} \mathrm{C}\left\{{ }^{1} \mathrm{H}\right\} \mathrm{NMR}(126 \mathrm{MHz})$ spectra of compound 16c $\left(\mathrm{CDCl}_{3}\right)$

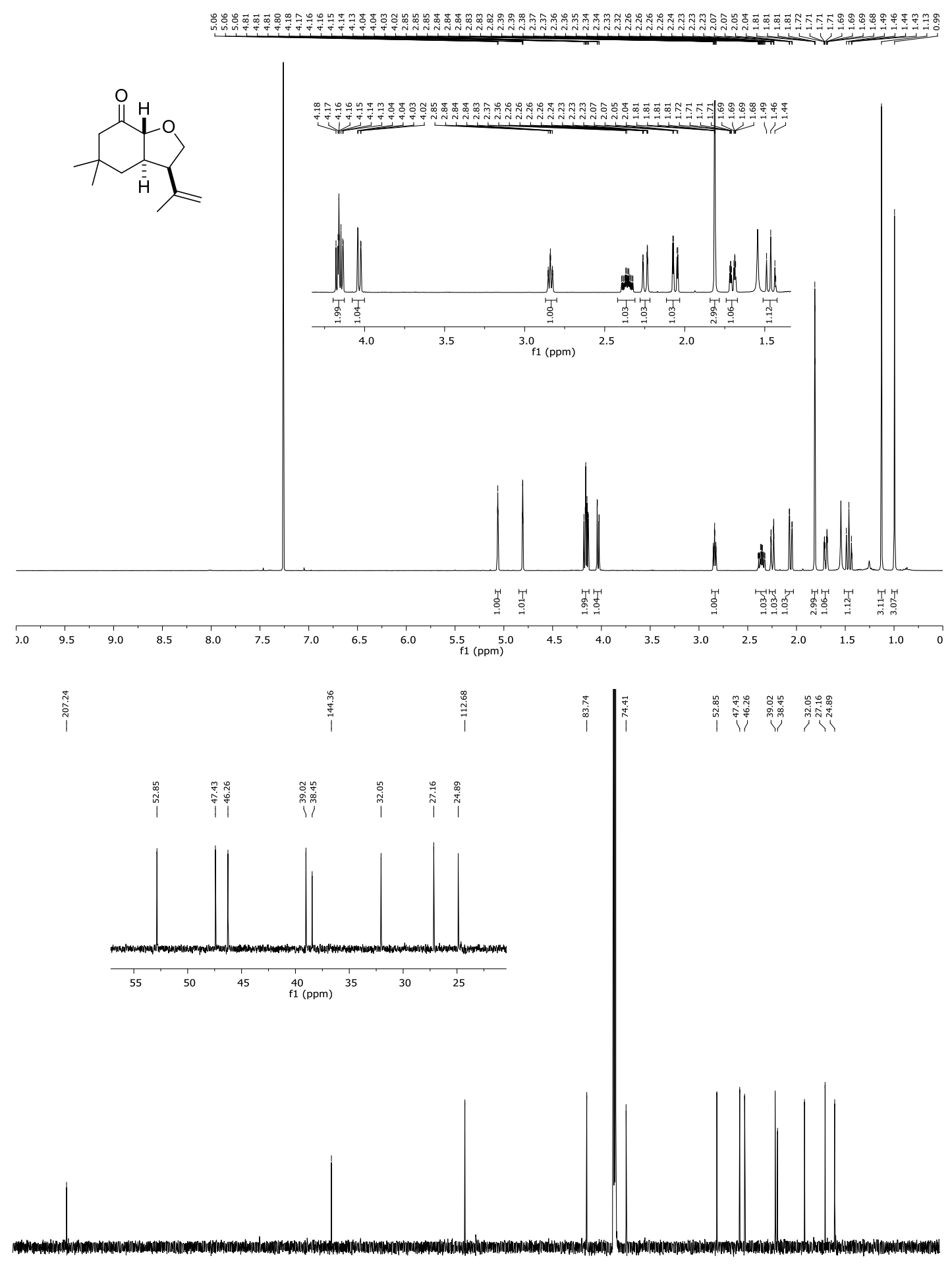

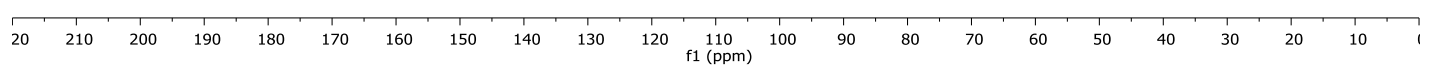




\section{${ }^{1} \mathrm{H}(400 \mathrm{MHz})$ and ${ }^{13} \mathrm{C}\left\{{ }^{1} \mathrm{H}\right\}$ NMR (101 MHz) spectra of compound $17\left(\mathrm{CDCl}_{3}\right)$}

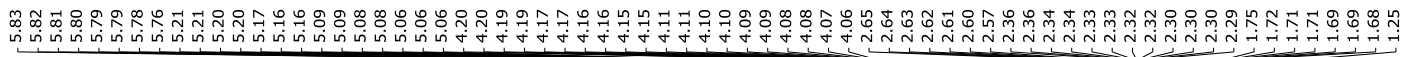<smiles>C=CCOC1(OCC)C2(C)CCC(C2)OC1(OC=C)OC=C</smiles>
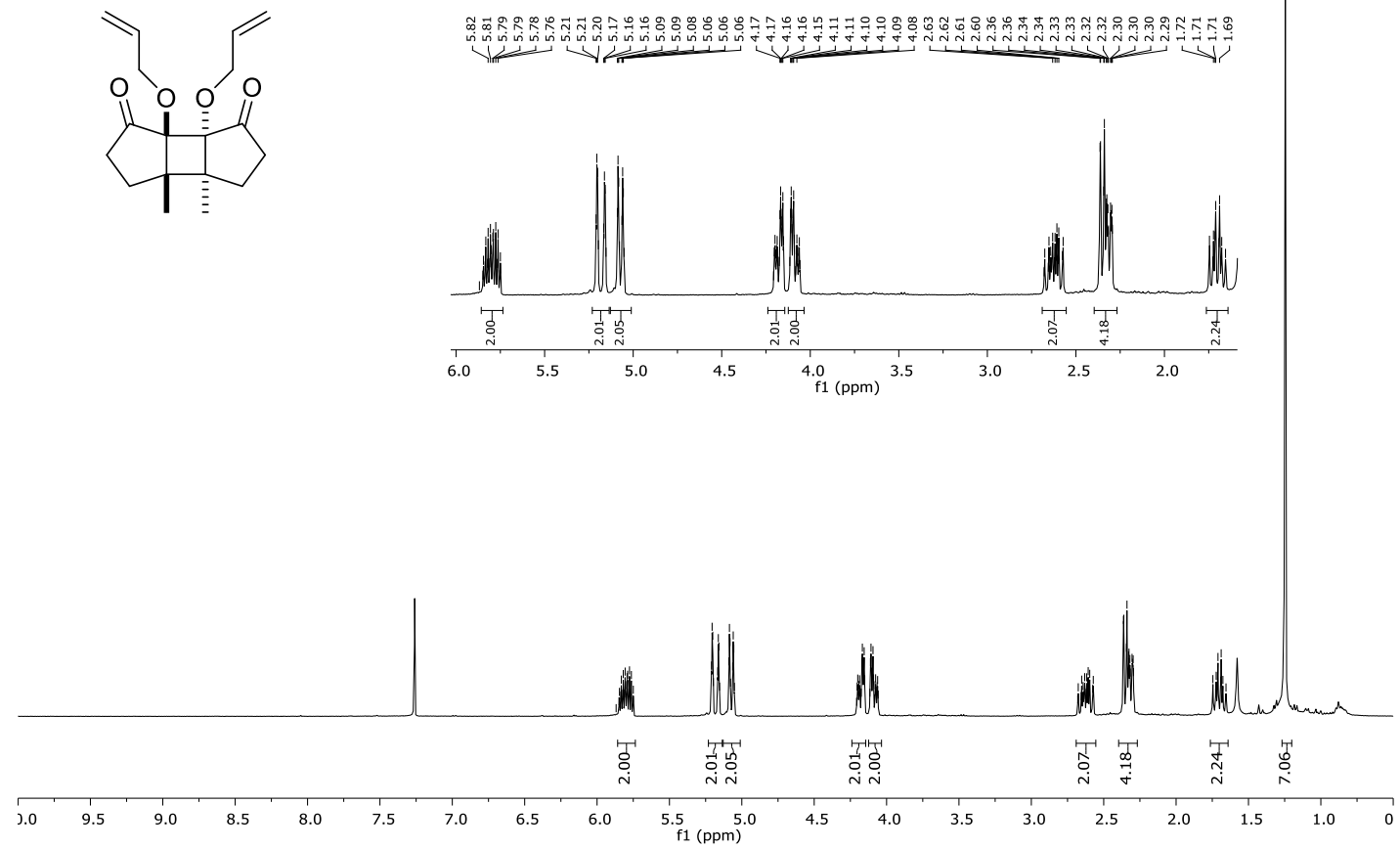

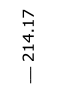

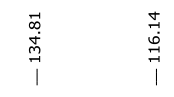

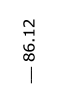

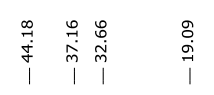

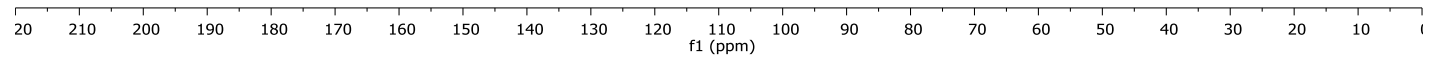

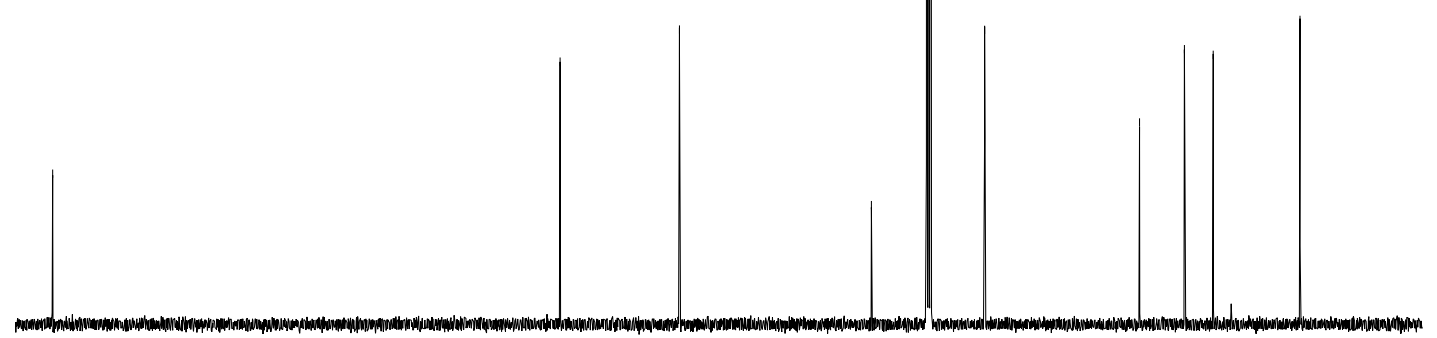


${ }^{1} \mathrm{H}(400 \mathrm{MHz})$ and ${ }^{13} \mathrm{C}\left\{{ }^{1} \mathrm{H}\right\} \mathrm{NMR}(126 \mathrm{MHz})$ spectra of compound $18\left(\mathrm{CDCl}_{3}\right)$

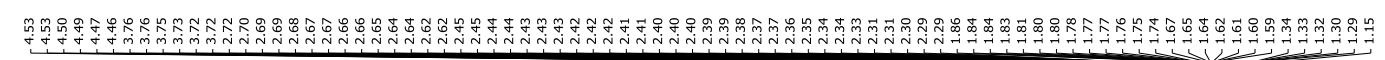
$\underbrace{0}_{-\infty}$
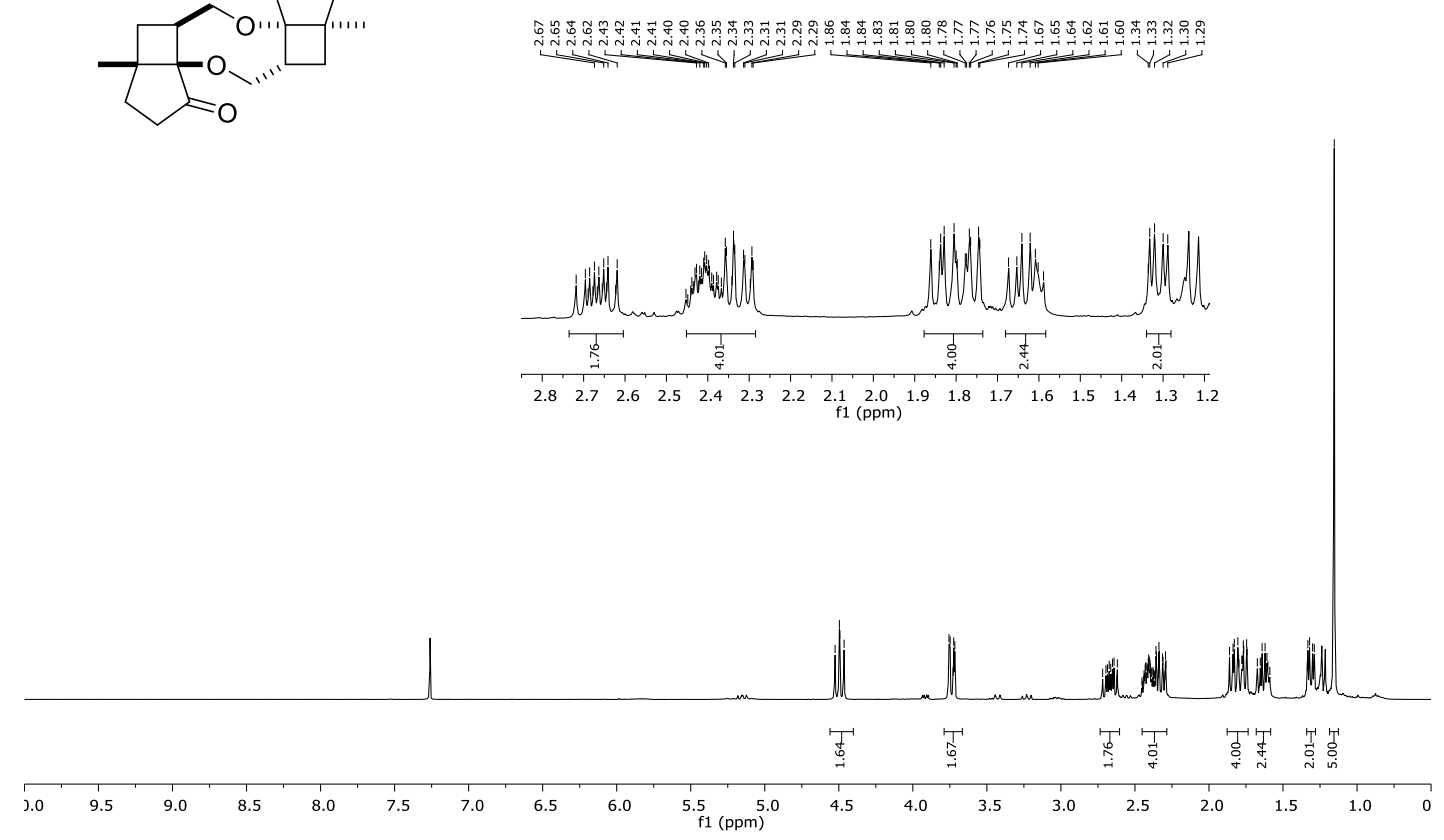
1
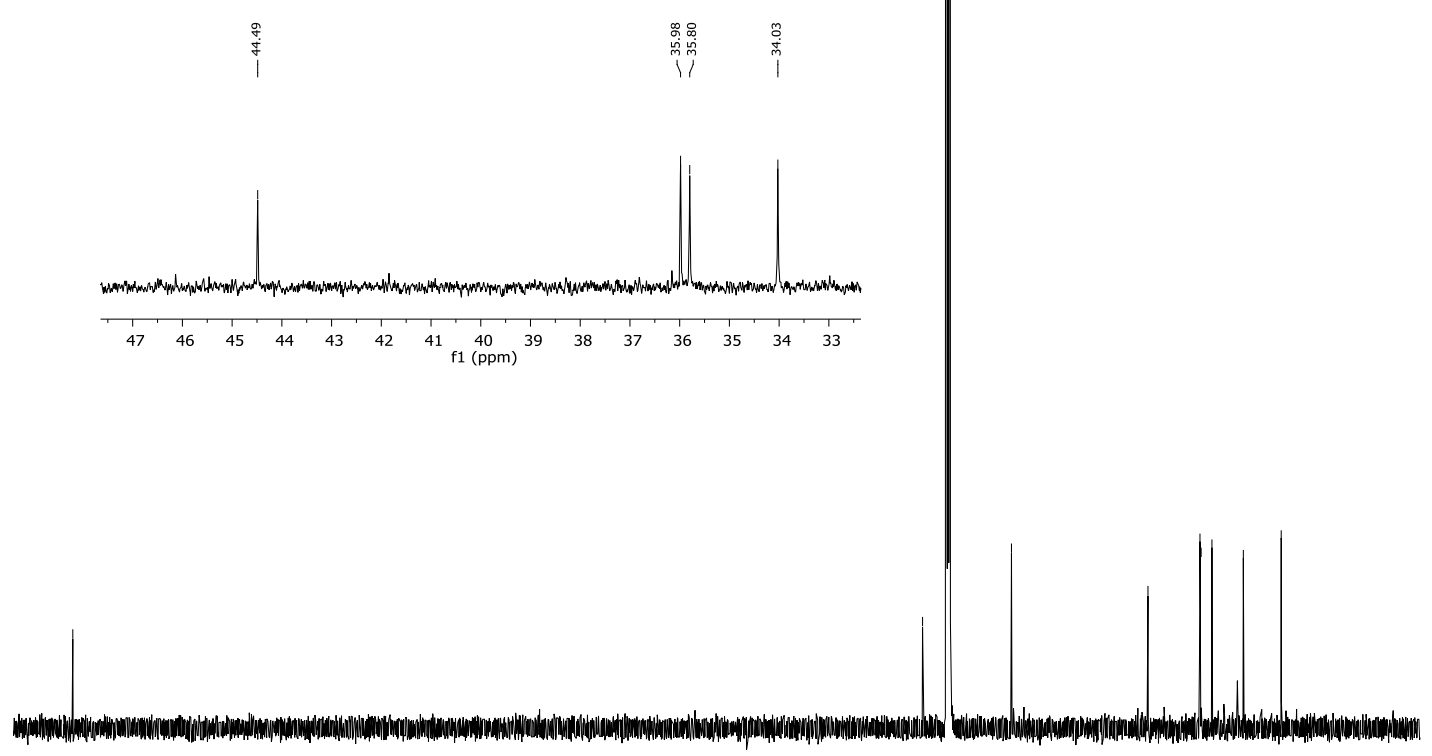

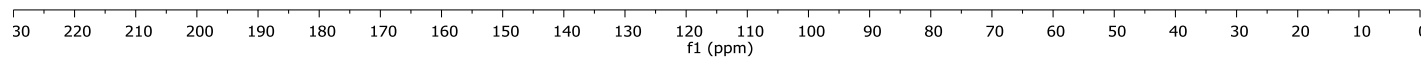


${ }^{1} \mathrm{H}(500 \mathrm{MHz})$ and ${ }^{13} \mathrm{C}\left\{{ }^{1} \mathrm{H}\right\}$ NMR $(126 \mathrm{MHz})$ spectra of compound $19\left(\mathrm{CDCl}_{3}\right)$

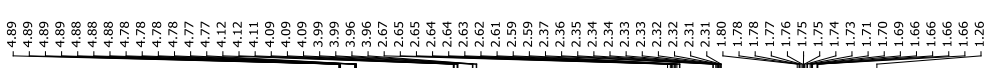
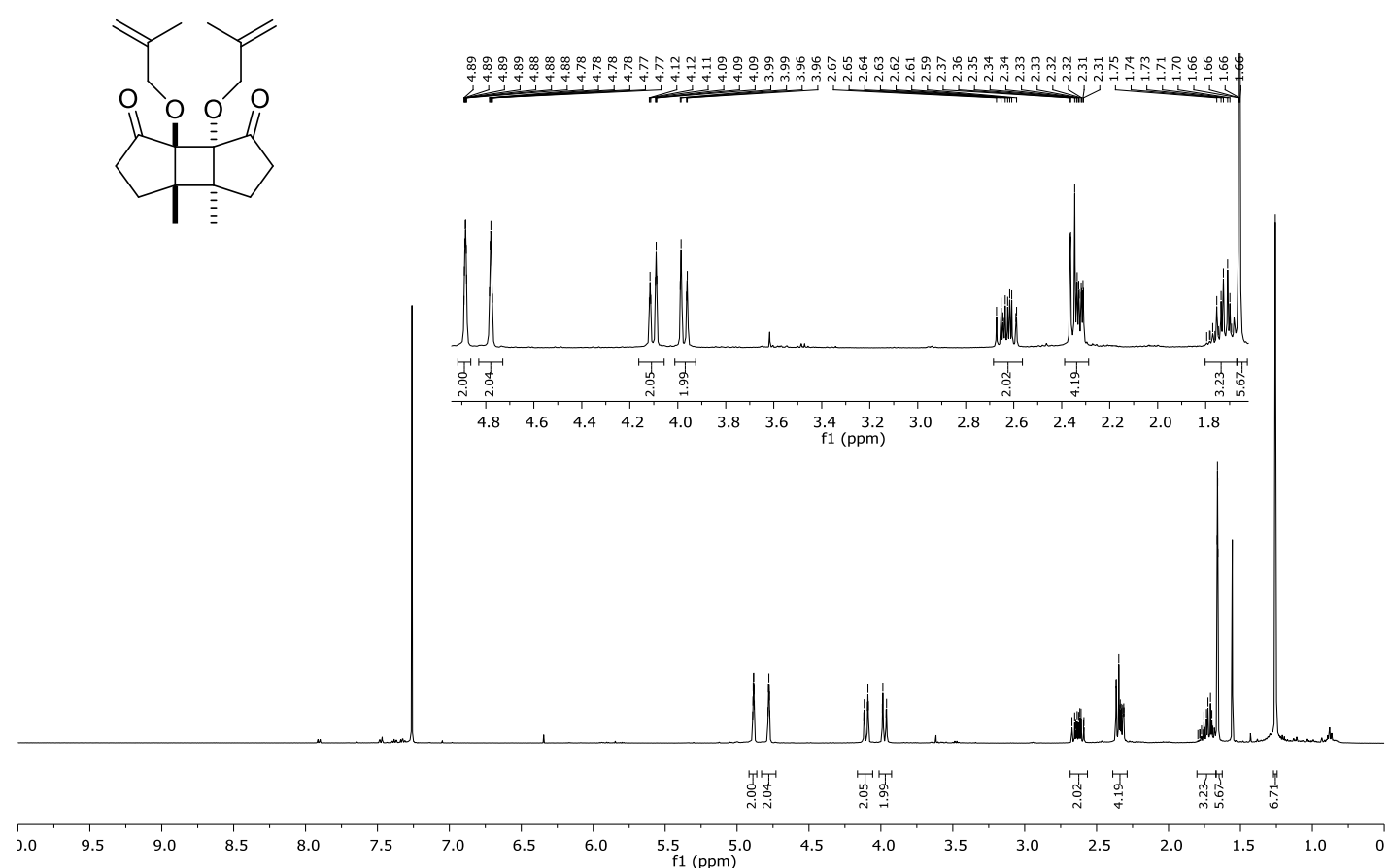

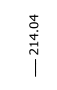
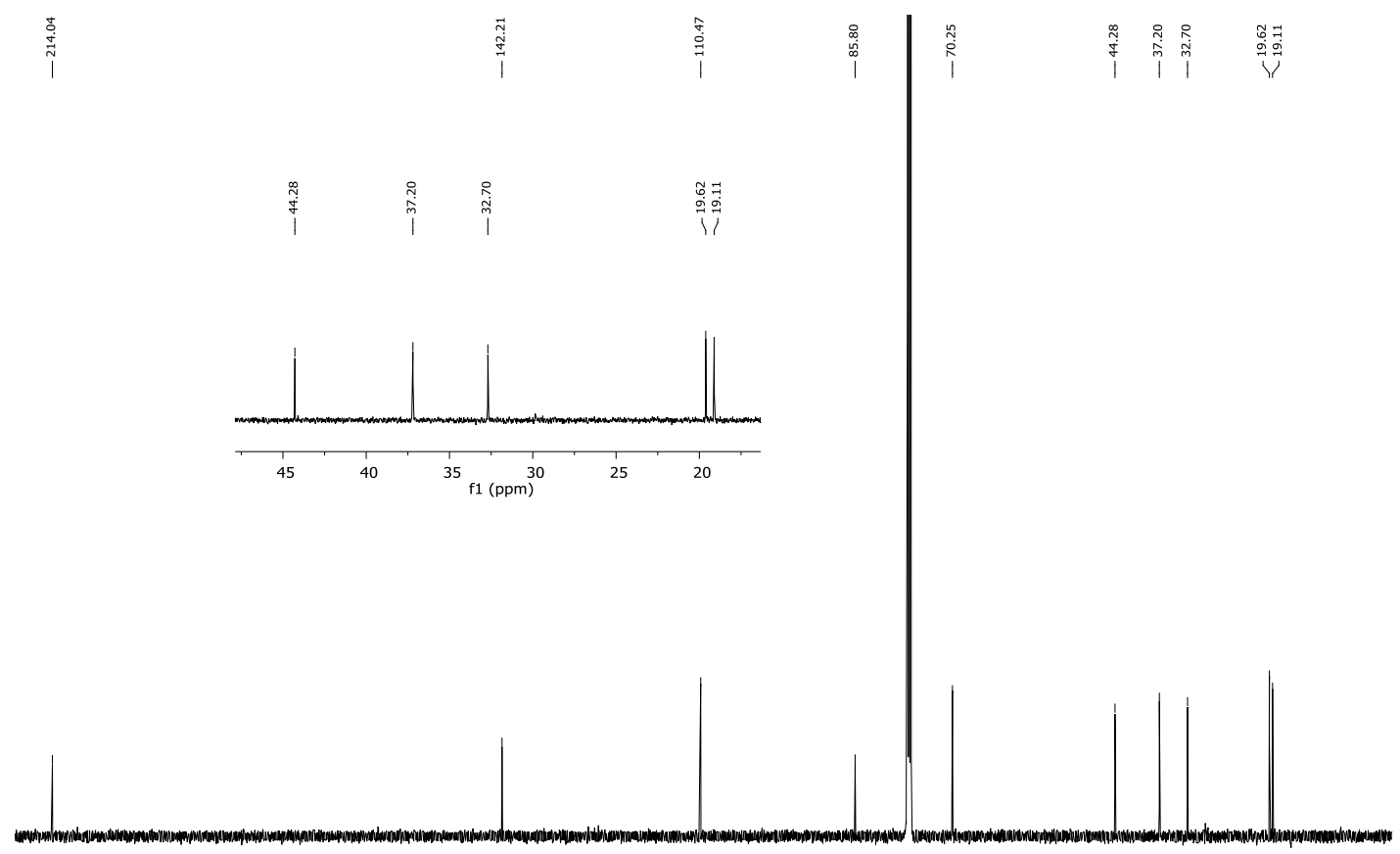

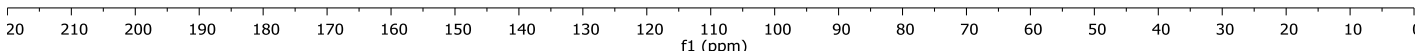


${ }^{1} \mathrm{H}(500 \mathrm{MHz})$ and ${ }^{13} \mathrm{C}\left\{{ }^{1} \mathrm{H}\right\} \mathrm{NMR}(126 \mathrm{MHz})$ spectra of compound $20\left(\mathrm{CDCl}_{3}\right)$
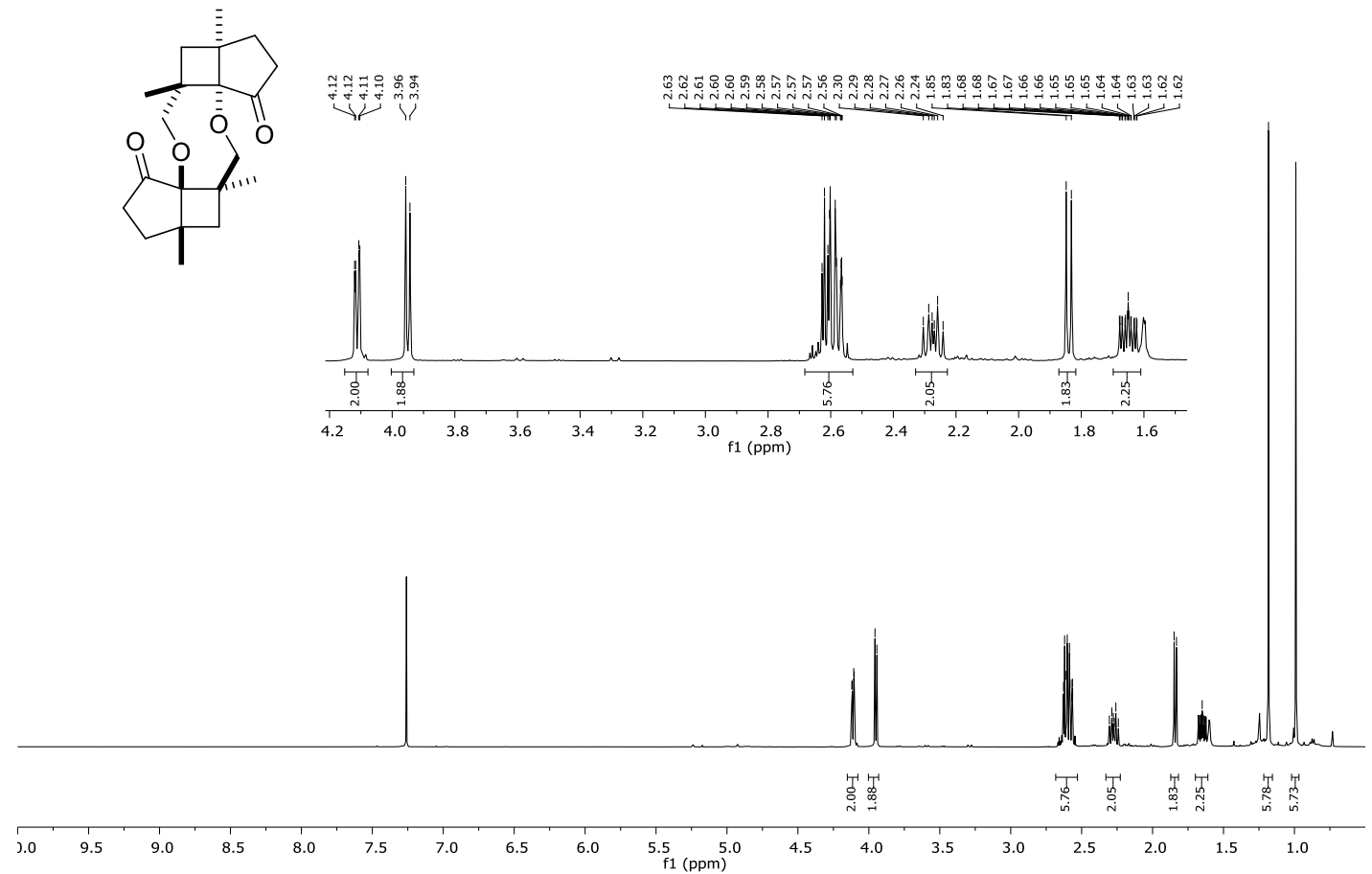
| 
${ }^{1} \mathrm{H}(500 \mathrm{MHz})$ and ${ }^{13} \mathrm{C}\left\{{ }^{1} \mathrm{H}\right\} \mathrm{NMR}(75 \mathrm{MHz})$ spectra of compound $21\left(\mathrm{CDCl}_{3}\right)$
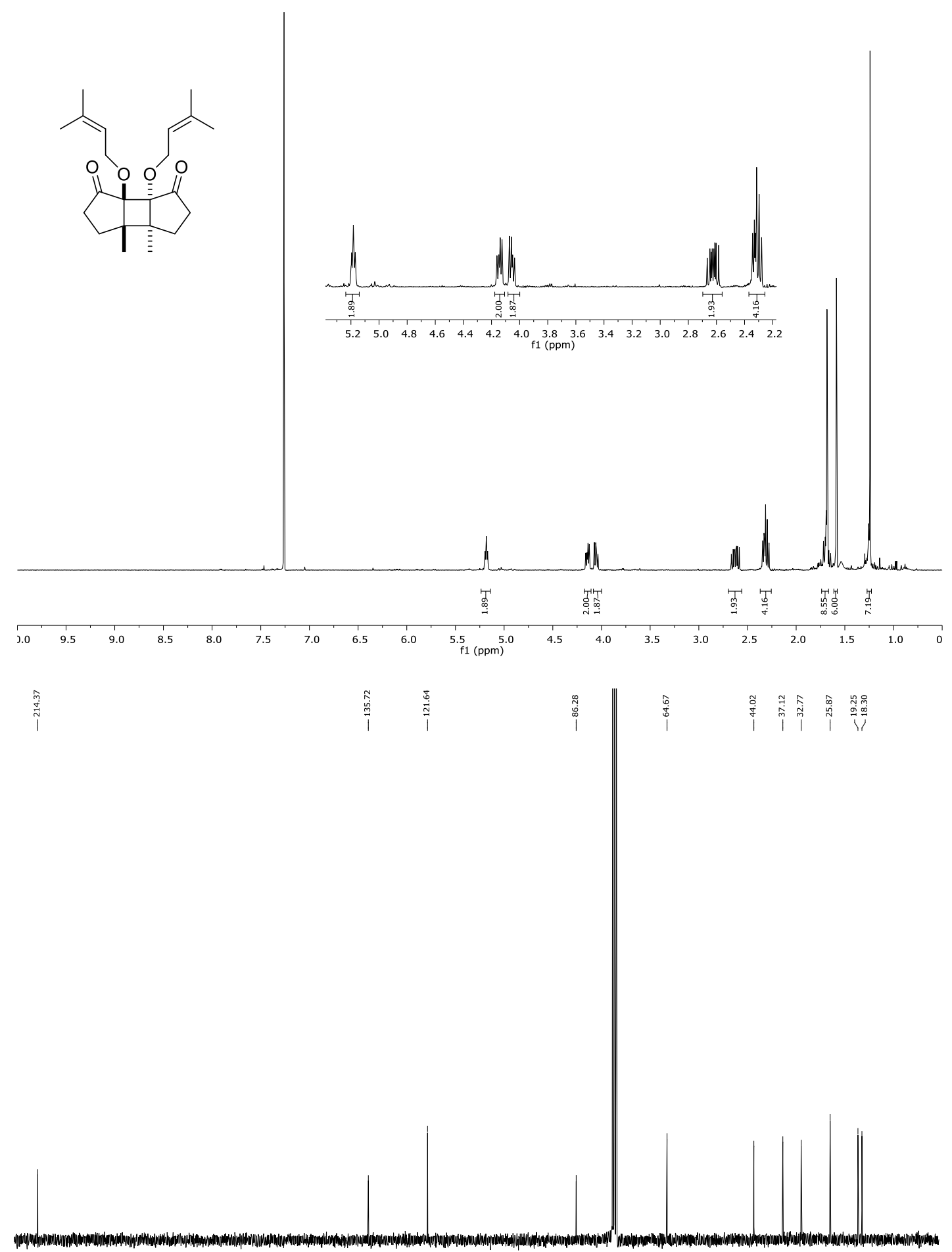

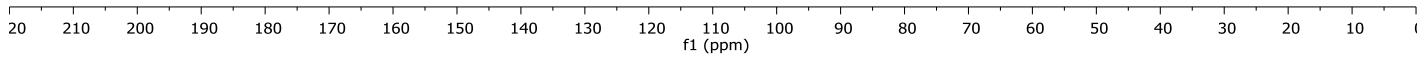


7. UV/vis Spectra of All Irradiation Precursors

UV/vis spectrum of compound $\mathbf{1 a}\left(0.5 \mathrm{mM}, \mathrm{CH}_{2} \mathrm{Cl}_{2} ; 50 \mathrm{~mm}, \mathrm{CH}_{2} \mathrm{Cl}_{2}\right)$

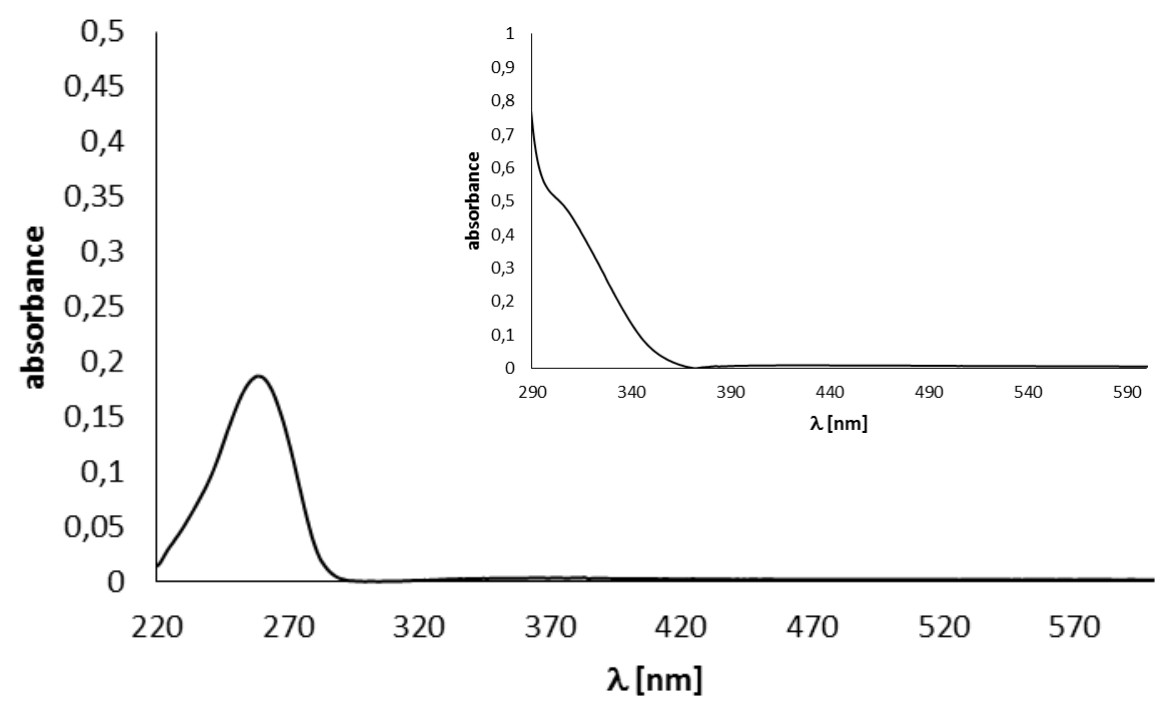

$\mathrm{UV} /$ vis spectrum of compound $\mathbf{1 b}\left(0.5 \mathrm{mM}, \mathrm{CH}_{2} \mathrm{Cl}_{2} ; 20 \mathrm{mM}, \mathrm{CH}_{2} \mathrm{Cl}_{2}\right)$

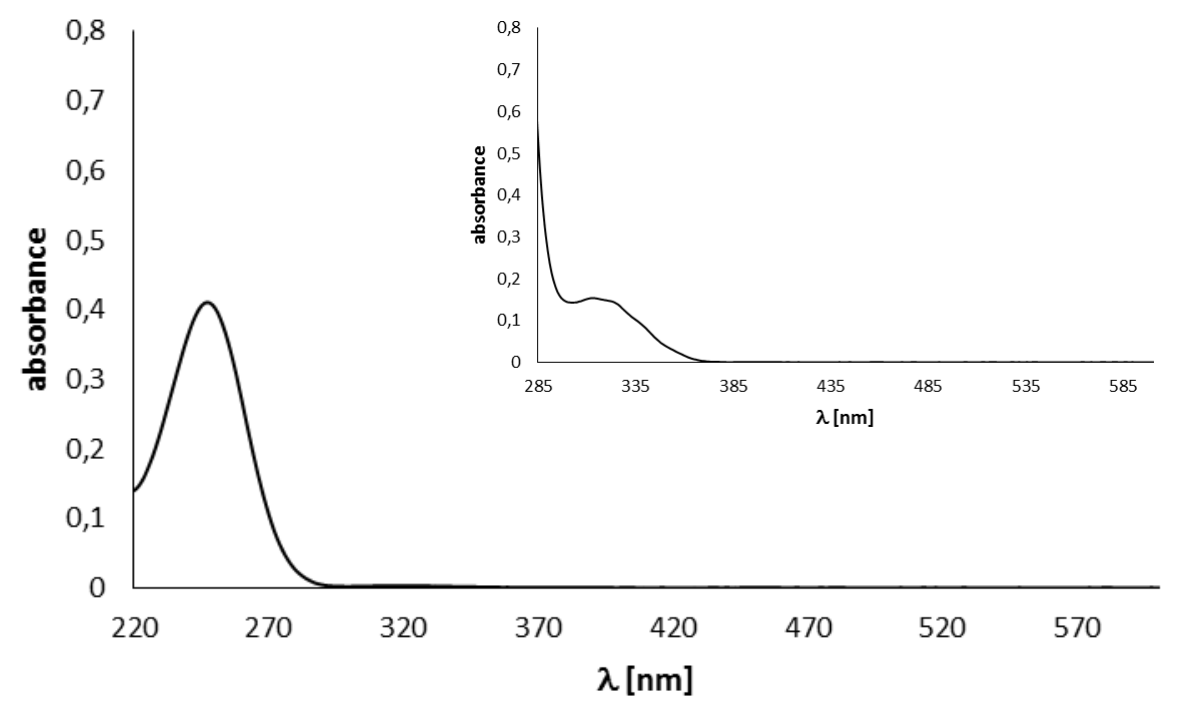


UV/vis spectrum of compound 1 c $\left(0.5 \mathrm{mM}, \mathrm{CH}_{2} \mathrm{Cl}_{2} ; 20 \mathrm{mM}, \mathrm{CH}_{2} \mathrm{Cl}_{2}\right)$

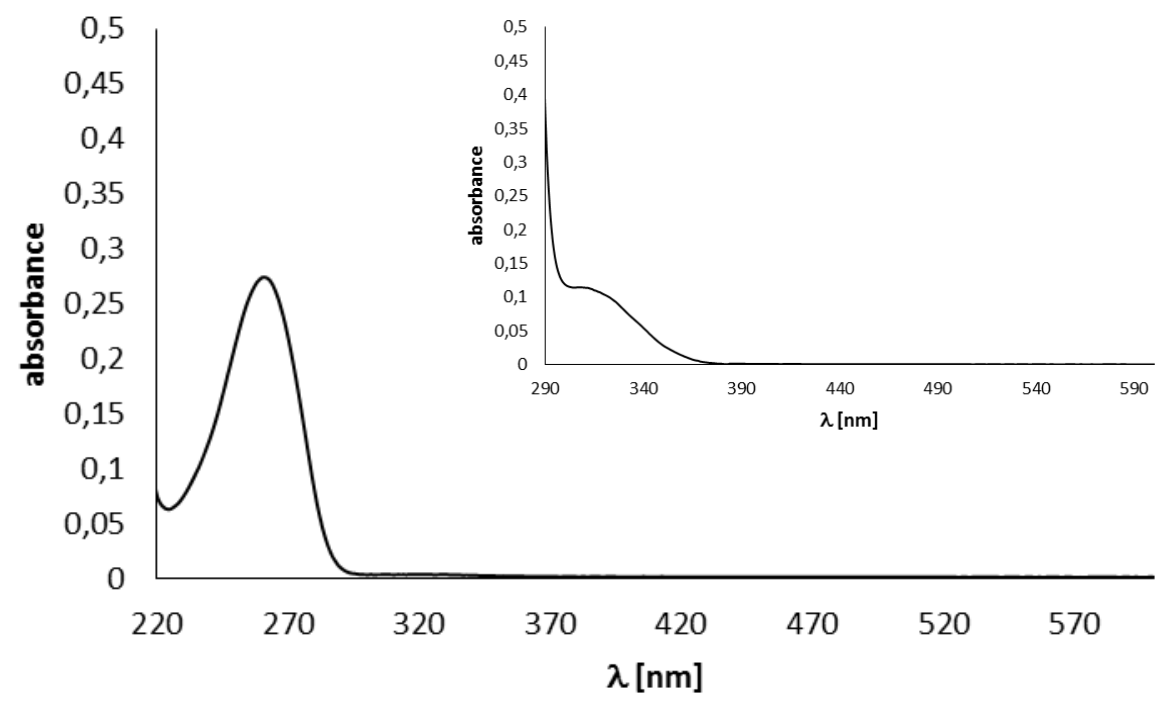

$\mathrm{UV} /$ vis spectrum of compound $\mathbf{1 d}\left(0.5 \mathrm{mM}, \mathrm{CH}_{2} \mathrm{Cl}_{2} ; 20 \mathrm{mM}, \mathrm{CH}_{2} \mathrm{Cl}_{2}\right)$

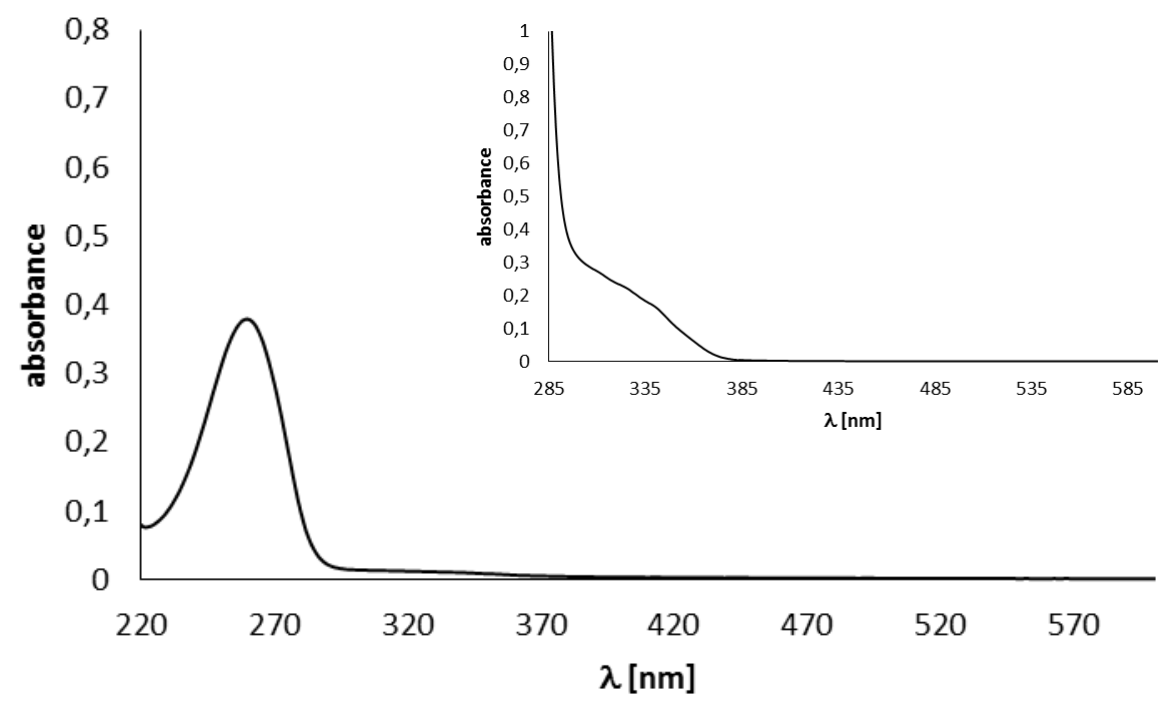


UV/vis spectrum of compound $\mathbf{1 e}\left(0.5 \mathrm{mM}, \mathrm{CH}_{2} \mathrm{Cl}_{2} ; 20 \mathrm{mM}, \mathrm{CH}_{2} \mathrm{Cl}_{2}\right)$

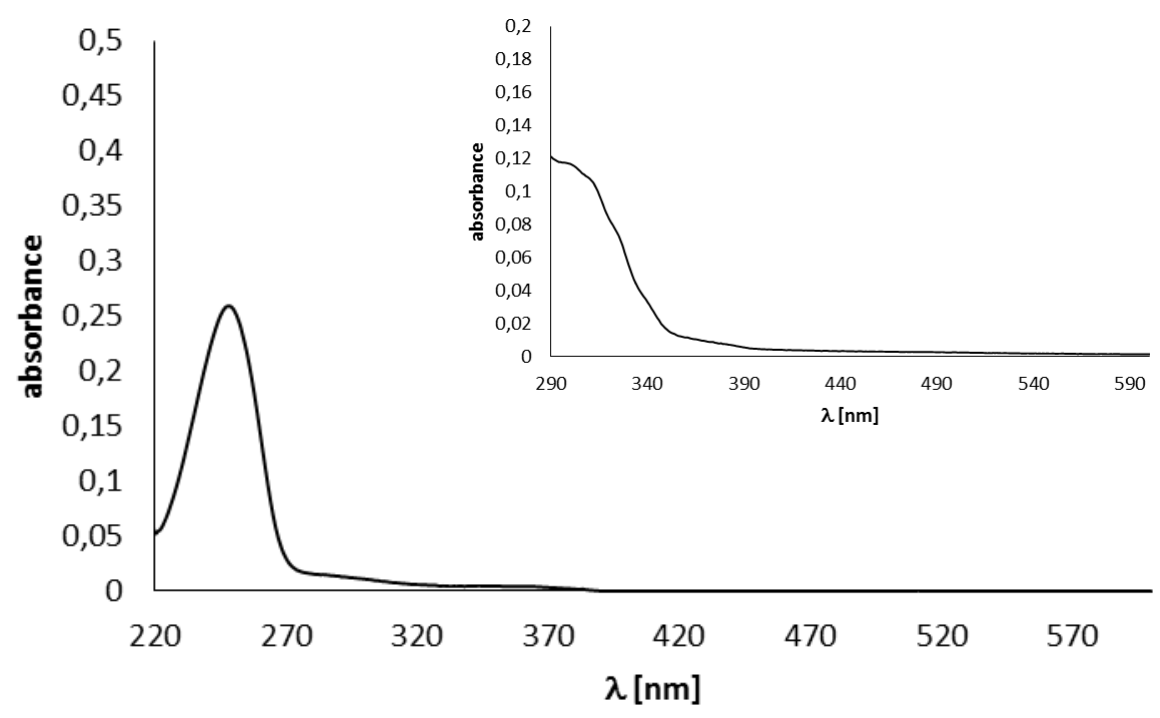

$\mathrm{UV} /$ vis spectrum of compound $\mathbf{1 f}\left(0.5 \mathrm{mM}, \mathrm{CH}_{2} \mathrm{Cl}_{2} ; 20 \mathrm{mM}, \mathrm{CH}_{2} \mathrm{Cl}_{2}\right)$

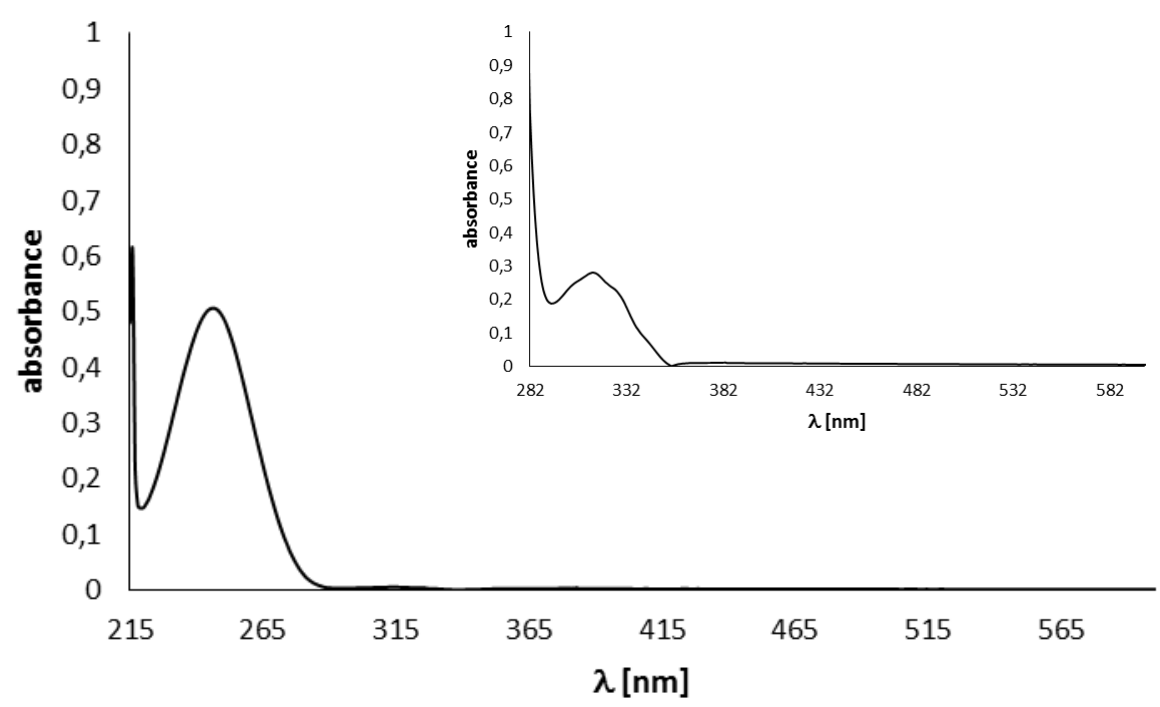


$\mathrm{UV} /$ vis spectrum of compound $\mathbf{2 a}$ (left) and $\mathbf{2 b}$ (right) $\left(0.5 \mathrm{mM}, \mathrm{CH}_{2} \mathrm{Cl}_{2}\right)$
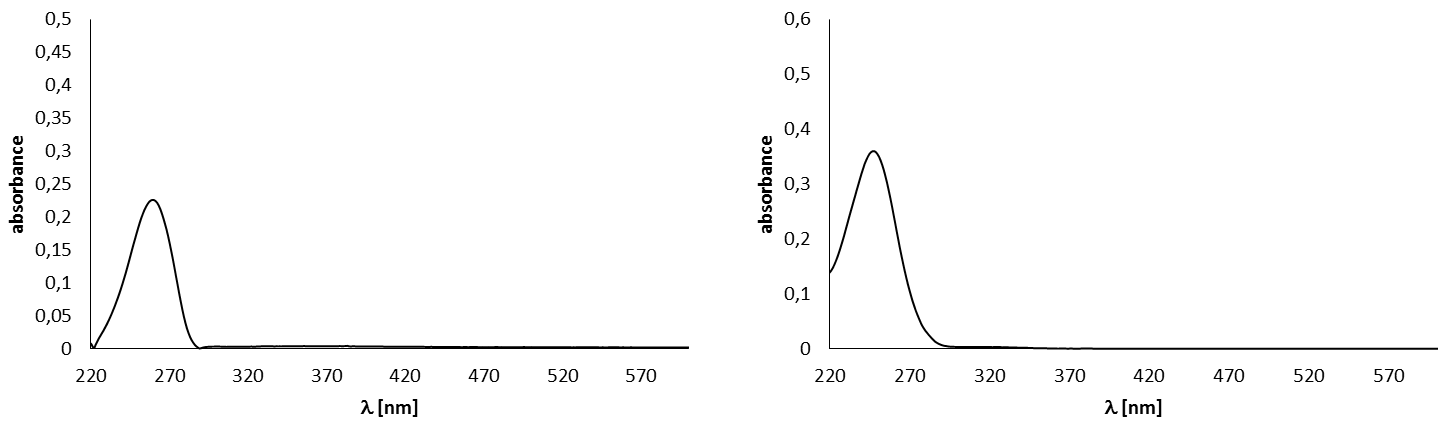

$\mathrm{UV} /$ vis spectrum of compound $\mathbf{2 c}$ (left) and $\mathbf{2 d}$ (right) $\left(0.5 \mathrm{mM}, \mathrm{CH}_{2} \mathrm{Cl}_{2}\right)$
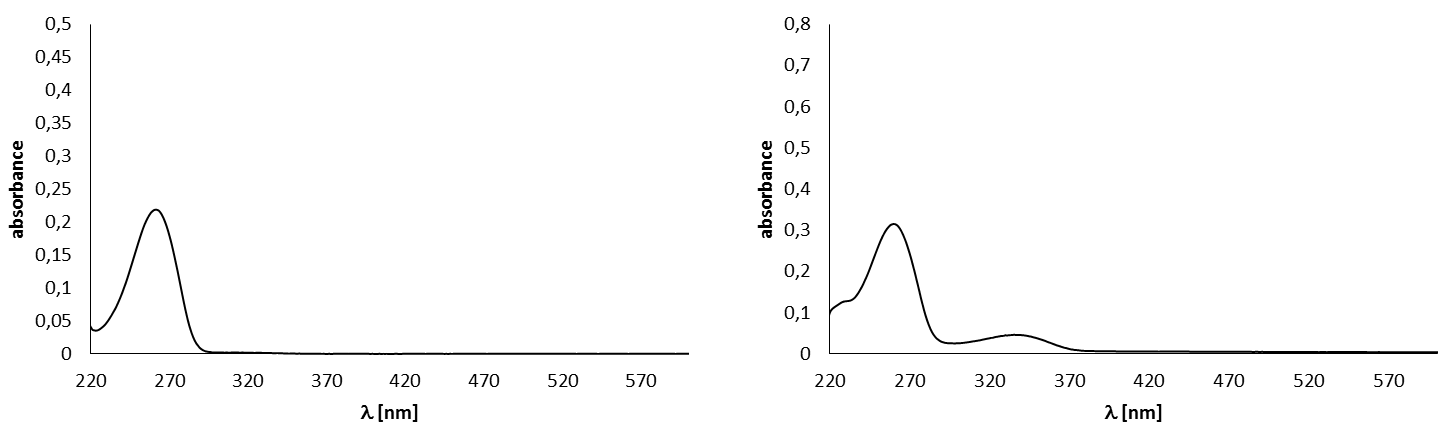

$\mathrm{UV} /$ vis spectrum of compound $2 \mathbf{e}$ (left) and $\mathbf{2 f}$ (right) $\left(0.5 \mathrm{mM}, \mathrm{CH}_{2} \mathrm{Cl}_{2}\right)$
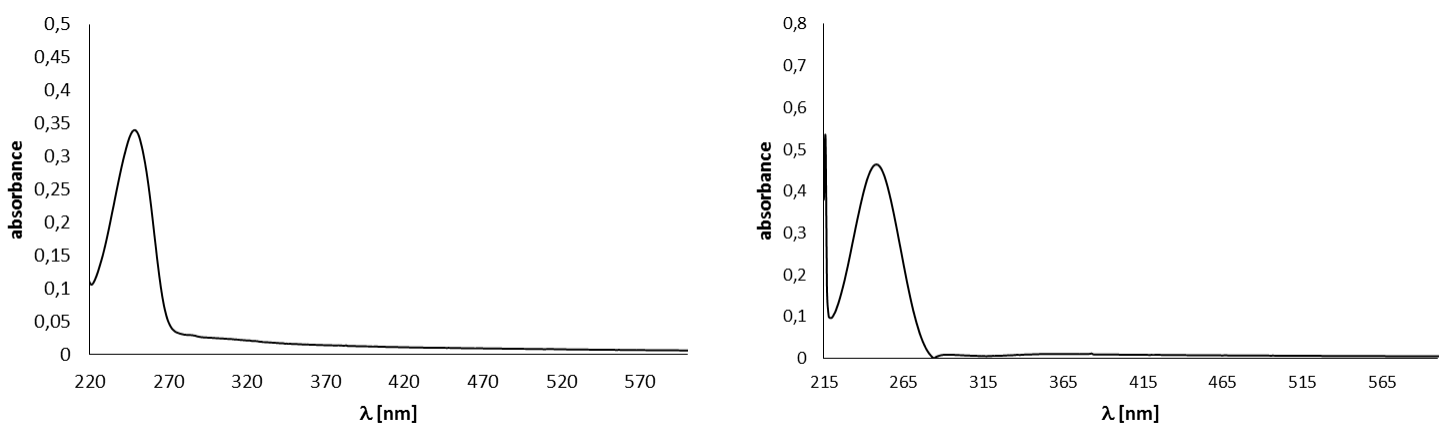
$\mathrm{UV} /$ vis spectrum of compound $\mathbf{3 a}$ (left) and $\mathbf{3 b}$ (right) $\left(0.5 \mathrm{mM}, \mathrm{CH}_{2} \mathrm{Cl}_{2}\right)$
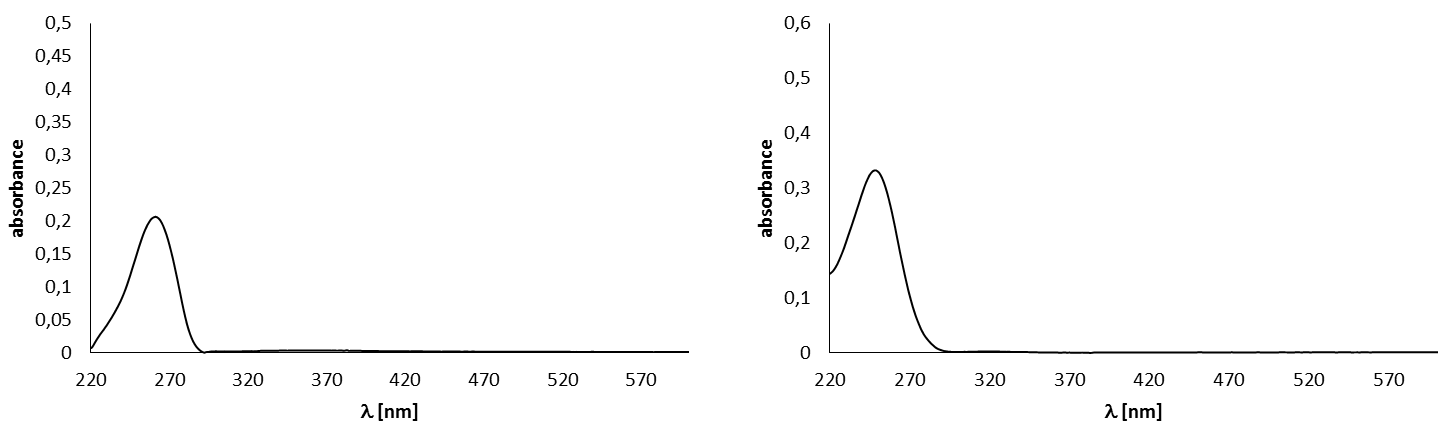

$\mathrm{UV} /$ vis spectrum of compound $\mathbf{3 c}$ (left) and $\mathbf{3 e}$ (right) $\left(0.5 \mathrm{mM}, \mathrm{CH}_{2} \mathrm{Cl}_{2}\right)$
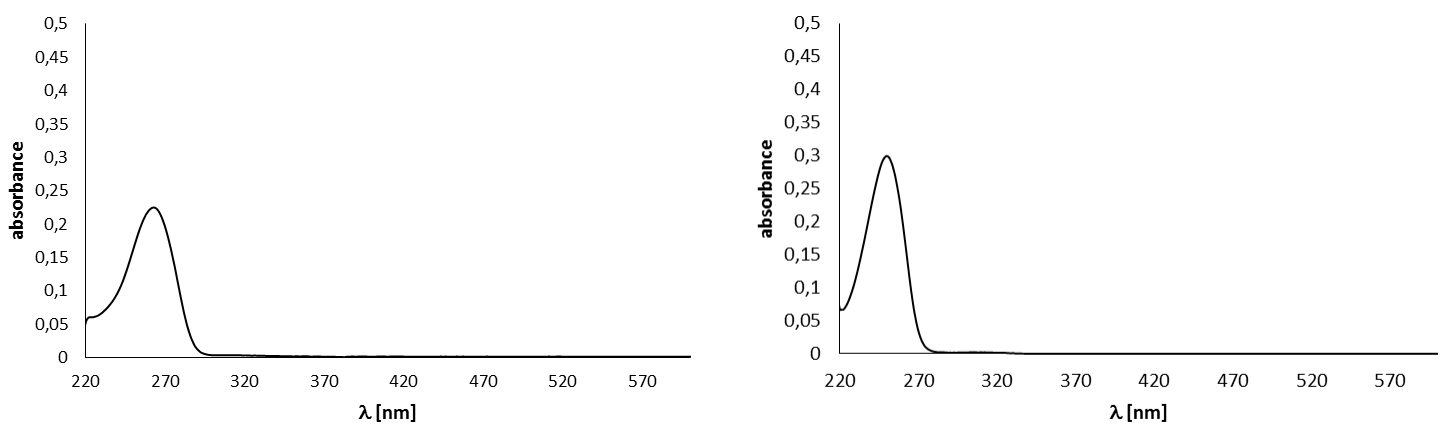

$\mathrm{UV} /$ vis spectrum of compound $\mathbf{3 f}$ (left) and $E-\mathbf{1 0} / Z$-10 (right) $\left(0.5 \mathrm{mM}, \mathrm{CH}_{2} \mathrm{Cl}_{2}\right)$
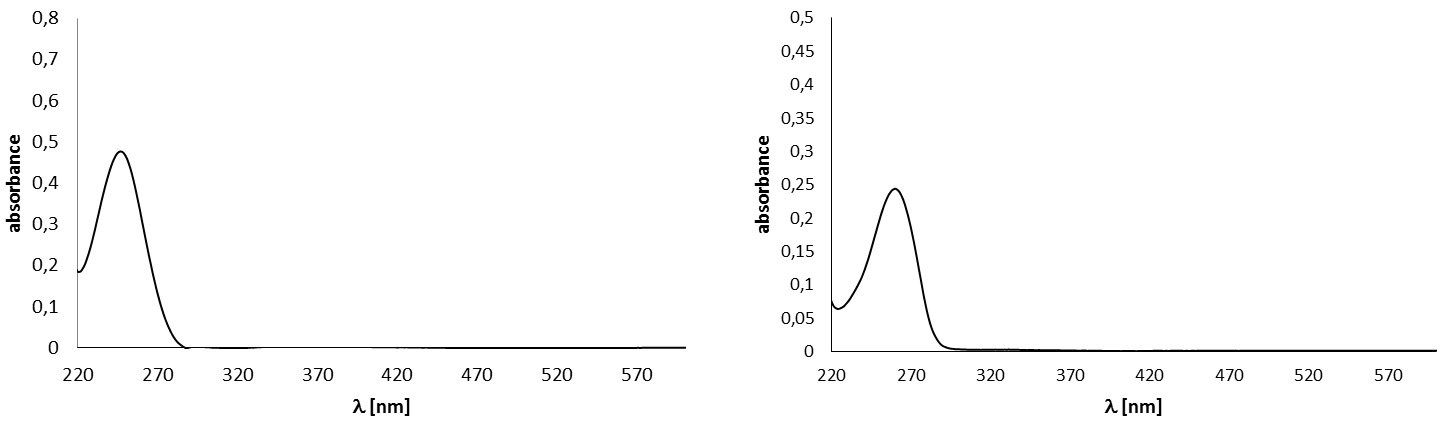
$\mathrm{UV} /$ vis spectrum of compound $E-\mathbf{1 2}$ (left) and Z-12 (right) $\left(0.5 \mathrm{mM}, \mathrm{CH}_{2} \mathrm{Cl}_{2}\right)$
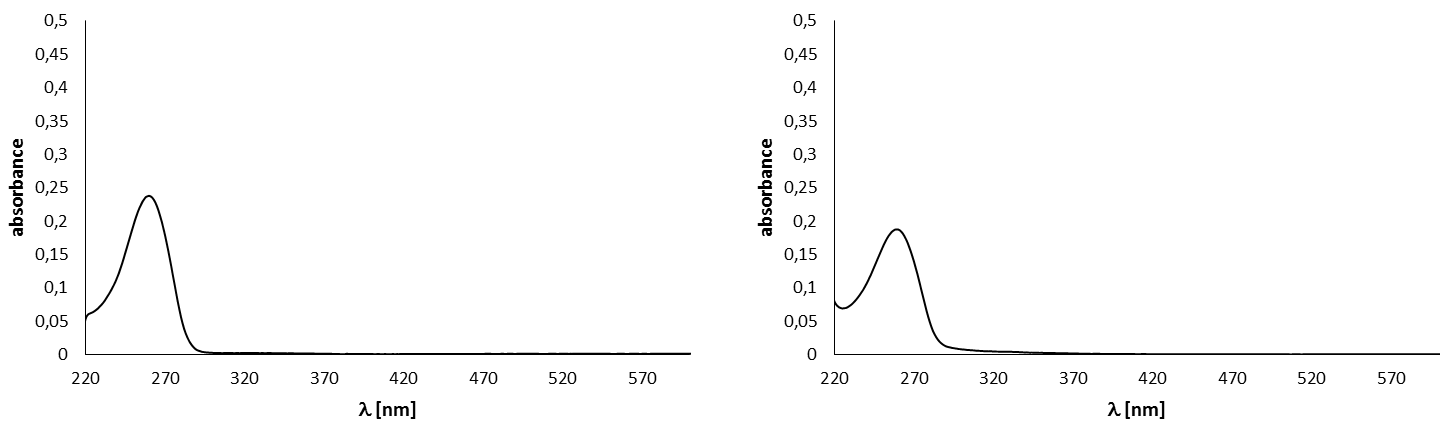


\section{X-ray Crystallographic Details}

Data were collected on Bruker D8 Venture single crystal x-ray diffractometer equipped with a CPAD detector (Bruker Photon II) (compounds 18, S1) or a CMOS detector (Bruker Photon-100) (compound 21), an IMS micro source (compounds 18, S1) or a TXS rotating anode (compound 21) with $\mathrm{MoK}_{\alpha}$ radiation $(\lambda=0.71073 \AA)$ and a Helios optic using the APEX3 software package. ${ }^{6}$ Measurements were performed on single crystals coated with perfluorinated ether. The crystals were fixed on top of a kapton micro sampler and frozen under a stream of cold nitrogen. A matrix scan was used to determine the initial lattice parameters. Reflections were corrected for Lorentz and polarisation effects, scan speed, and background using SAINT. ${ }^{7}$ Absorption correction, including odd and even ordered spherical harmonics was performed using SADABS. ${ }^{7}$ Space group assignments were based upon systematic absences, E statistics, and successful refinement of the structures. The structures were solved using SHELXT with the aid of successive difference Fourier maps, and were refined against all data using SHELXL in conjunction with SHELXLE. ${ }^{8,9,10}$ Hydrogen atoms were calculated in ideal positions as follows: Methyl hydrogen atoms were refined as part of rigid rotating groups, with a $\mathrm{C}-\mathrm{H}$ distance of $0.98 \AA$ and $\mathrm{U}_{\text {iso(H) }}=$ 1.5. $\mathrm{U}_{\mathrm{eq}(\mathrm{C})}$. Other $\mathrm{H}$ atoms were placed in calculated positions and refined using a riding model, with methylene and aromatic $\mathrm{C}-\mathrm{H}$ distances of $0.99 \AA$ and $0.95 \AA$, respectively, other $\mathrm{C}-\mathrm{H}$ distances of $1.00 \AA$, all with $\mathrm{U}_{\mathrm{iso}(\mathrm{H})}=1.2 \cdot \mathrm{U}_{\mathrm{eq}(\mathrm{C})}$. Non-hydrogen atoms were refined with anisotropic displacement parameters. Full-matrix least-squares refinements were carried out by minimizing $\Sigma w\left(\mathrm{~F}_{\mathrm{o}}{ }^{2}-\mathrm{F}_{\mathrm{c}}{ }^{2}\right)^{2}$ with the SHELXL weighting scheme. ${ }^{8}$ Neutral atom scattering factors for all atoms and anomalous dispersion corrections for the non-hydrogen atoms were taken from International Tables for Crystallography. ${ }^{11}$ Images of the crystal structures were generated with Mercury (main article) and PLATON (SI). ${ }^{12,13}$ CCDC 2010591-2010593 contains the supplementary crystallographic data for this paper. These data are provided free of charge by The Cambridge Crystallographic Data Centre. 


\section{Compound 18 (CCDC 2010593)}

Single crystals were obtained by solvent evaporation from a saturated solution of 18 in $\mathrm{Et}_{2} \mathrm{O}$.

Ellipsoids in the ORTEP structure are displayed at the 50\% probability level.

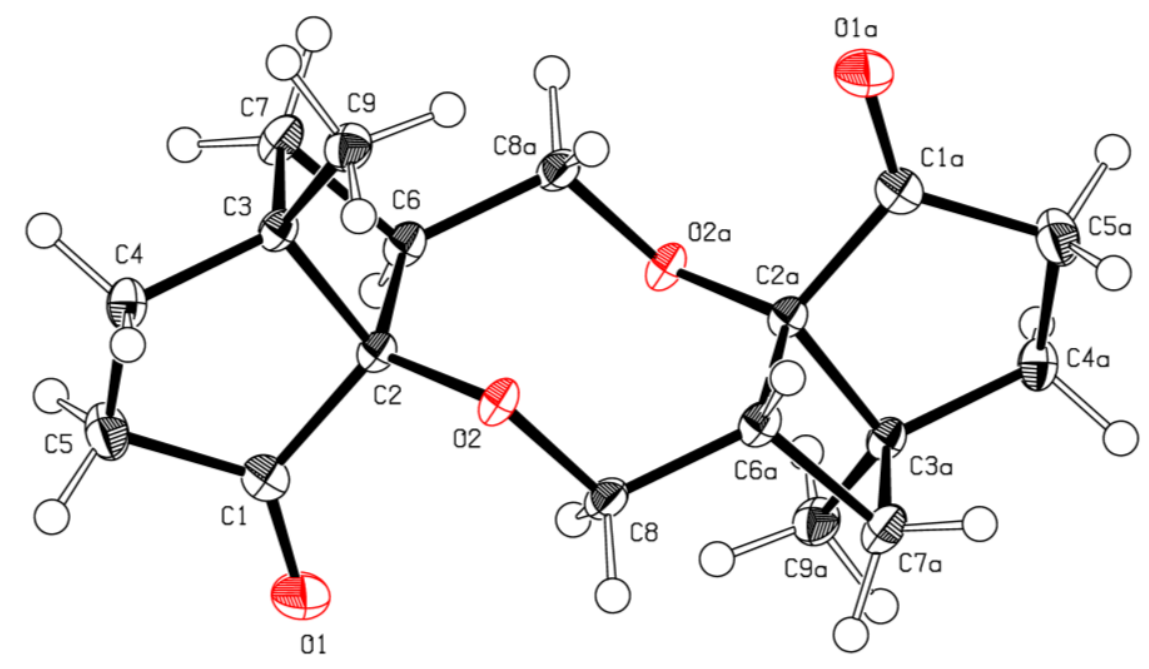

Diffractometer operator C. Jandl scanspeed 1-10 s per frame

dx $37 \mathrm{~mm}$

3415 frames measured in 13 data sets

phi-scans with delta_phi $=0.5$

omega-scans with delta_omega $=0.5$

shutterless mode

\section{Crystal data}

$\underline{\mathrm{C}_{18} \mathrm{H}_{24} \mathrm{O}_{4}}$

$M_{r}=\underline{304.37}$

$D_{\mathrm{x}}=\underline{1.320} \mathrm{Mg} \mathrm{m}^{-3}$

$\underline{\text { Monoclinic, }} \underline{C 2 / c}$

Melting point: $>493 \mathrm{~K}$

Hall symbol: $\underline{-\mathrm{C} 2 \mathrm{yc}}$

$\underline{\text { Mo } K \alpha}$ radiation, $\lambda=\underline{0.71073} \AA$

$a=\underline{18.1075(17)} \AA$

Cell parameters from 9504 reflections

$b=\underline{11.052(1) \AA}$

$\theta=\underline{2.2}-\underline{28.3^{\circ}}$

$c=\underline{8.2387(8)} \AA$

$\mu=\underline{0.09} \mathrm{~mm}^{-1}$

$\beta=\underline{111.686(3)^{\circ}}$

$T=\underline{100} \mathrm{~K}$ 
$V=\underline{1532.1(3)} \AA^{3}$

$Z=\underline{4}$

$F(000)=\underline{656}$

\section{Data collection}

Bruker D8 Venture

diffractometer

Radiation source: IMS microsource $\underline{1720}$ reflections with $\underline{I>2 \sigma(I)}$

Helios optic monochromator $\quad R_{\text {int }}=\underline{0.029}$

Detector resolution: $\underline{7.5}$ pixels $\mathrm{mm}^{-1} \theta_{\max }=\underline{27.9^{\circ}}, \theta_{\min }={\underline{2.2^{\circ}}}^{\circ}$

phi- and $\omega$-rotation scans

Absorption correction: $\underline{\text { multi-scan }} \quad k=\underline{-14} \underline{14}$

$\underline{\text { SADABS 2016/2, Bruker }}$

$T_{\min }=\underline{0.725}, T_{\max }=\underline{0.746}$

$l=\underline{-10} \underline{10}$

$\underline{49708}$ measured reflections

\section{Refinement}

Refinement on $\underline{F^{2}}$

Least-squares matrix: $\underline{\text { full }}$

$R\left[F^{2}>2 \sigma\left(F^{2}\right)\right]=\underline{0.034}$

$w R\left(F^{2}\right)=\underline{0.093}$

$S=\underline{1.09}$

$\underline{1827}$ reflections

101 parameters

$\underline{0}$ restraints

$\underline{0}$ constraints

Primary atom site location: intrinsic phasing
Secondary atom site location: difference Fourier map

Hydrogen site location: inferred from neighbouring sites

$\underline{\mathrm{H} \text {-atom parameters constrained }}$

$\mathrm{W}=1 /\left[\Sigma^{2}\left(F \mathrm{O}^{2}\right)+(0.0421 P)^{2}+1.3923 P\right]$

WHERE $P=\left(F \mathrm{O}^{2}+2 F \mathrm{C}^{2}\right) / 3$

$(\Delta / \sigma)_{\max } \leq 0.001$

$\Delta \rho_{\max }=\underline{0.39} \mathrm{e}^{-3}$

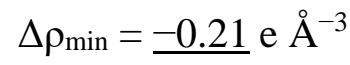

Extinction correction: none

Extinction coefficient: = 


\section{Compound 21 (CCDC 2010591)}

Single crystals were obtained by diffusion of pentane into a saturated solution of 21 in $\mathrm{CH}_{2} \mathrm{Cl}_{2}$. Ellipsoids in the ORTEP structure are displayed at the 50\% probability level.

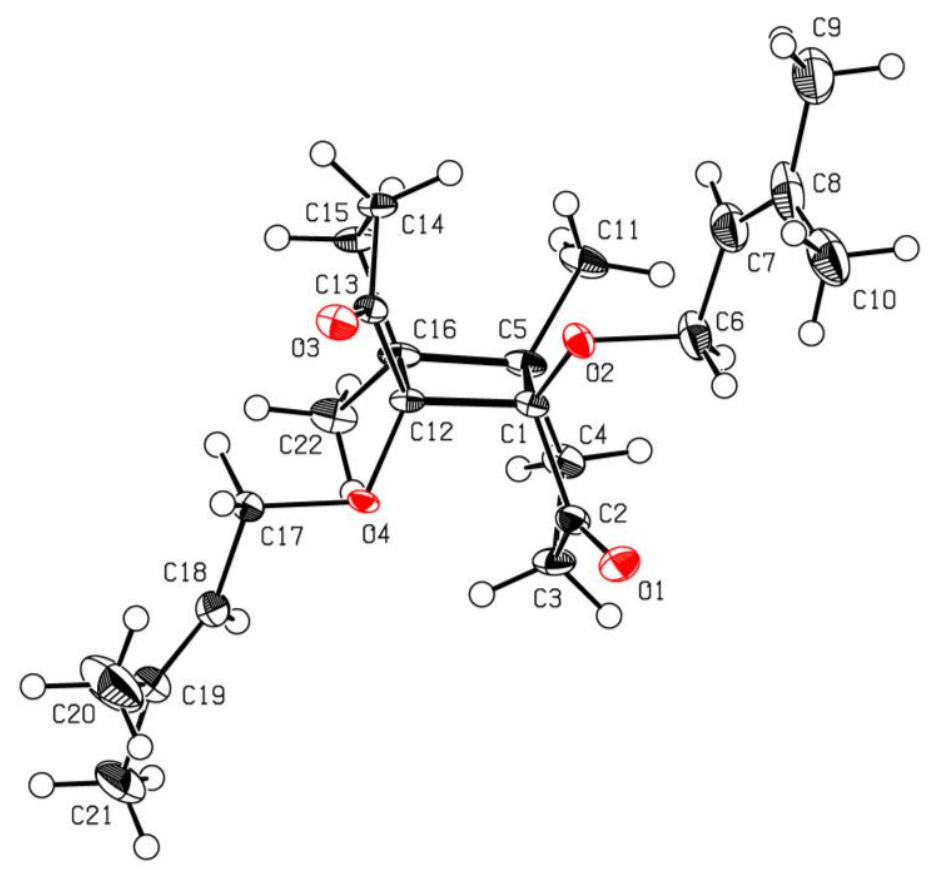

Diffractometer operator C. Jandl scanspeed 10-60 s per frame dx $50 \mathrm{~mm}$ 1488 frames measured in 6 data sets phi-scans with delta_phi $=0.5$ omega-scans with delta_omega $=0.5$ shutterless mode

Crystal data

$\underline{\mathrm{C}}_{22} \underline{\mathrm{H}}_{32} \underline{\mathrm{O}}_{4}$

$M_{r}=\underline{360.47}$

$D_{\mathrm{x}}=\underline{1.204} \mathrm{Mg} \mathrm{m}^{-3}$

Monoclinic, $\underline{P 2} 1 / n$

Melting point: $\underline{358}-\underline{371} \mathrm{~K}$

Hall symbol: $\underline{-\mathrm{P} 2 \mathrm{yn}}$

$\underline{\text { Mo } K \alpha}$ radiation, $\lambda=\underline{0.71073} \AA$

$a=\underline{11.8018(14)} \AA$

Cell parameters from $\underline{8914}$ reflections

$b=\underline{6.7440(8)} \AA$

$\theta=\underline{3.1}^{-25.5^{\circ}}$

$c=\underline{25.405(3)} \AA$

$\mu=\underline{0.08} \mathrm{~mm}^{-1}$

$\beta=\underline{100.493(4)^{\circ}}$

$T=\underline{100} \mathrm{~K}$

$V=\underline{1988.2(4)} \AA^{3}$

$\underline{\text { Fragment, }} \underline{\text { colourless }}$ 
$F(000)=\underline{784}$

Data collection

Bruker D8 Venture

diffractometer

$\underline{3647}$ independent reflections

Radiation source: TXS rotating anode $\underline{2817}$ reflections with $\underline{I>2 \sigma(I)}$

Helios optic monochromator $\quad R_{\text {int }}=\underline{0.066}$

Detector resolution: $\underline{7.5}$ pixels $\mathrm{mm}^{-1}$

$\theta_{\max }=\underline{25.4^{\circ}}, \theta_{\min }=\underline{2.7^{\circ}}$

phi- and $\omega-$-rotation scans

$h=\underline{-14} \underline{14}$

Absorption correction: multi-scan

$k=\underline{-8} \quad \underline{7}$

$\underline{S A D A B S} 2016 / 2$, Bruker

$T_{\min }=\underline{0.647}, T_{\max }=\underline{0.745}$

$l=\underline{-28} \underline{30}$

$\underline{28402}$ measured reflections

Refinement

Refinement on $\underline{F^{2}}$

Least-squares matrix: $\underline{\text { full }}$

$R\left[F^{2}>2 \sigma\left(F^{2}\right)\right]=\underline{0.065}$

$w R\left(F^{2}\right)=\underline{0.188}$

$S=\underline{1.10}$

$\underline{3647}$ reflections

$\underline{489}$ parameters

$\underline{602}$ restraints

$\underline{0}$ constraints

Primary atom site location: intrinsic phasing
Secondary atom site location: difference Fourier map

Hydrogen site location: inferred from neighbouring sites

$\underline{\mathrm{H} \text {-atom parameters constrained }}$

$\mathrm{W}=1 /\left[\Sigma^{2}\left(F \mathrm{O}^{2}\right)+(0.0982 P)^{2}+1.8965 P\right]$

WHERE $P=\left(F \mathrm{O}^{2}+2 F \mathrm{C}^{2}\right) / 3$

$(\Delta / \sigma)_{\max } \leq 0.001$

$\Delta \rho_{\max }=\underline{0.47} \mathrm{e} \AA^{-3}$

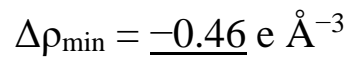

Extinction correction: none

Extinction coefficient: = 


\section{Compound S1 (CCDC 2010592)}

Single crystals were obtained by solvent evaporation from a saturated solution of $\mathbf{S 1}$ in $\mathrm{CH}_{2} \mathrm{Cl}_{2}$. Ellipsoids in the ORTEP structure are displayed at the 50\% probability level.

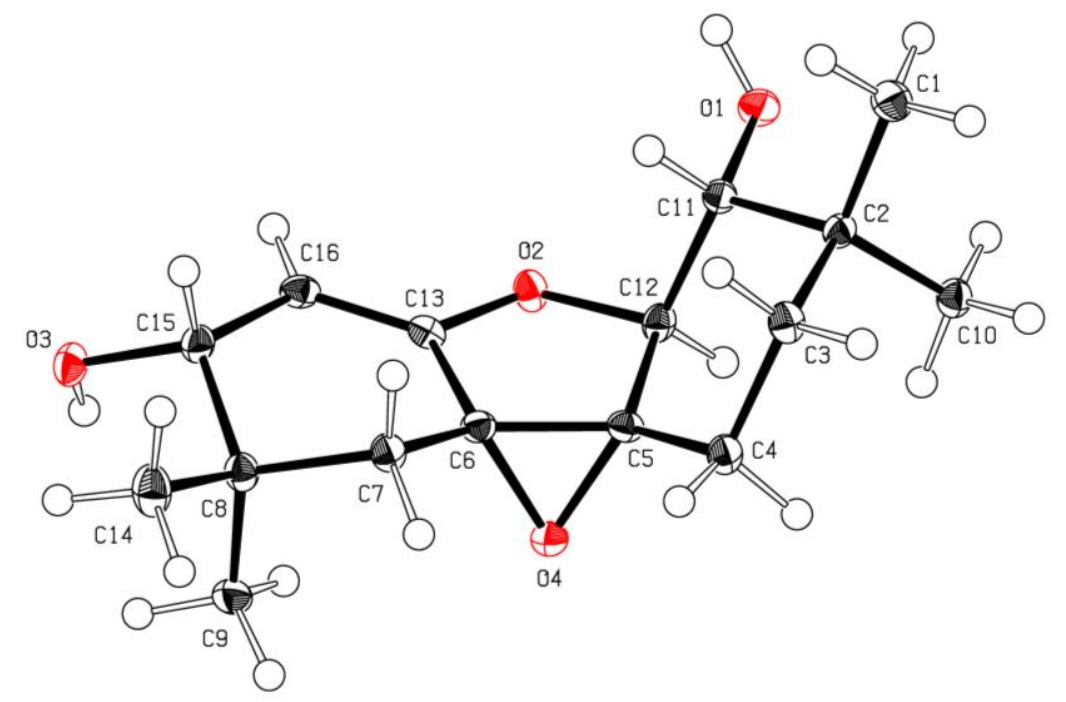

Diffractometer operator T. Pickl \& A. Poethig

phi-scans with delta_phi $=0.5$

omega-scans with delta_omega $=0.5$

shutterless mode

Crystal data

$\underline{\mathrm{C}}_{16} \underline{\mathrm{H}}_{24} \underline{\mathrm{O}}_{4}$

$M_{r}=\underline{280.35}$

$D_{\mathrm{x}}=\underline{1.291} \mathrm{Mg} \mathrm{m}^{-3}$

Monoclinic, $\underline{P 2_{1} / n}$

Hall symbol: $\underline{-\mathrm{P} 2 \mathrm{yn}}$

$\underline{\text { Mo } K \alpha}$ radiation, $\lambda=\underline{0.71073} \AA$

$a=\underline{6.1182(3)} \AA$

Cell parameters from $\underline{6447}$ reflections

$b=\underline{10.6025(5)} \AA$

$\theta=\underline{2.7}-\underline{26.4^{\circ}}$

$c=\underline{22.3764(12)} \AA$

$\mu=\underline{0.09} \mathrm{~mm}^{-1}$

$\beta=\underline{96.280(3)^{\circ}}$

$T=\underline{100} \mathrm{~K}$

$V=\underline{1442.81(13)} \AA^{3}$

Needle, clear colourless

$Z=\underline{4}$

$\underline{0.74} \times \underline{0.09} \times \underline{0.06} \mathrm{~mm}$ 
$F(000)=\underline{608}$

\section{Data collection}

Bruker D8 Venture

diffractometer

Radiation source: IMS microsource

HELIOS optic monochromator

Detector resolution: $\underline{7.5}$ pixels $\mathrm{mm}^{-1}$

phi-and $\omega$-rotation scans

Absorption correction: multi-scan

$\underline{S A D A B S} 2016 / 2$, Bruker

$T_{\min }=\underline{0.655}, T_{\max }=\underline{0.745}$

$\underline{28565}$ measured reflections

Refinement

Refinement on $\underline{F^{2}}$

Least-squares matrix: full

$R\left[F^{2}>2 \sigma\left(F^{2}\right)\right]=\underline{0.053}$

$w R\left(F^{2}\right)=\underline{0.128}$

$S=\underline{1.14}$

2960 reflections

193 parameters

$\underline{0}$ restraints

$\underline{0}$ constraints

Primary atom site location: intrinsic phasing $\underline{2960}$ independent reflections

$\underline{2598}$ reflections with $\underline{I>2 \sigma(I)}$

$R_{\text {int }}=\underline{0.065}$

$\theta_{\max }={\underline{26.4^{\circ}}}^{\circ}, \theta_{\min }={\underline{2.1^{\circ}}}^{\circ}$

$h=\underline{-7} \underline{7}$

$k=\underline{-13} \quad \underline{13}$

$l=\underline{-27} \underline{27}$ 


\section{References}

${ }^{1}$ Clark, W. D. K.; Litt, A. D.; Steel, C. Triplet lifetimes of benzophenone, acetophenone, and triphenylene in hydrocarbons. J. Am. Chem. Soc. 1969, 91, 5413-5415.

${ }^{2}$ Iyer, A.; Clay, A.; Jockusch, S.; Sivaguru, J. Evaluating brominated thioxanthones as organophotocatalysts. J. Phys. Org. Chem. 2017, 30, e3738.

${ }^{3}$ (a) Baranoff, E.; Curchod, B. F. E.; Monti, F.; Steimer, F.; Accorsi, G.; Tavernelli, I.;

Rothlisberger, U.; Scopelliti, R.; Grätzel, M.; Nazeeruddin, M. K. Influence of Halogen Atoms on a Homologous Series of Bis-Cyclometalated Iridium(III) Complexes. Inorg. Chem. 2012, 51, 799-811. (b) Yi, S.; Kim, J.-H.; Cho, Y.-J.; Lee, J.; Choi, T.-S.; Cho, D. W.; Pac, C.; Han, W.-S.; Son, H.-J.; Kang, S. O. Stable Blue Phosphorescence Iridium(III) Cyclometalated Complexes Prompted by Intramolecular Hydrogen Bond in Ancillary Ligand. Inorg. Chem. 2016, 55, 33243331.

${ }^{4}$ Singh, A.; Teegardin, K.; Kelly,M.; Prasad, K. S.; Krishnan, S.; Weaver, J. D. Facile synthesis and complete characterization of homoleptic and heteroleptic cyclometalated Iridium(III) complexes for photocatalysis. J. Organomet. Chem. 2015, 776, 51-59.

5 (a) Juris, A.; Balzani, V.; Belser, P.; von Zelewsky, A. Characterization of the Excited State Properties of Some New Photosensitizers of the Ruthenium (Polypyridine) Family. Helv. Chim. Acta 1981, 64, 2175-2182. (b) Kalyanasundaram, K. Photophysics, photochemistry and solar energy conversion with tris(bipyridyl)ruthenium(II) and its analogues. Coord. Chem. Rev. 1982, 46, 159-244.

6 APEX suite of crystallographic software, APEX 3, Version 2016-9.0, Bruker AXS Inc., Madison, Wisconsin, USA, 2016.

${ }^{7}$ SAINT, Versions 8.37A and 8.38A and SADABS, Version 2016/2, Bruker AXS Inc., Madison, Wisconsin, USA, 2016/2017.

${ }^{8}$ Sheldrick, G.M. SHELXT - Integrated space-group and crystal-structure determination. Acta Crystallogr. Sect. A 2015, 71, 3-8.

${ }^{9}$ Sheldrick, G.M. Crystal structure refinement with SHELXL. Acta Crystallogr. Sect. C 2015, 71, 3-8. 
${ }^{10}$ Hübschle, C.B.; Sheldrick, G. M.; Dittrich, B. ShelXle: a Qt graphical user interface for SHELXL J. Appl. Cryst. 2011, 44, 1281-1284.

11 International Tables for Crystallography, Vol. C (Ed.: A. J. Wilson), Kluwer Academic Publishers, Dordrecht, The Netherlands, 1992, Tables 6.1.1.4 (pp. 500-502), 4.2.6.8 (pp. 219222), and 4.2.4.2 (pp. 193-199).

${ }^{12}$ Macrae, C. F.; Bruno, I. J.; Chisholm, J. A.; Edgington, P. R.; McCabe, P.; Pidcock, E.; Rodriguez-Monge, L.; Taylor, R.; van de Streek, J.; Wood, P. A. Mercury CSD 2.0 - new features for the visualization and investigation of crystal structures. J. Appl. Cryst. 2008, 41, 466470.

${ }^{13}$ Spek, A. L. Structure validation in chemical crystallography. Acta Crystallogr. Sect. D 2009, $65,148-155$. 